Alma Mater Studiorum - Università di Bologna

SCUOLA DI SCIENZE

Corso di Laurea Magistrale in Astrofisica e Cosmologia

Dipartimento di Fisica e Astronomia

\title{
MULTIFREQUENCY VLBA OBSERVATIONS OF TWO COMPACT SYMMETRIC OBJECTS
}

Tesi di Laurea Magistrale

Candidato:

Sara Rastello
Relatore:

Chiar.mo Prof.

Daniele Dallacasa

Co-Relatore:

Dott.sa Monica Orienti

Sessione I

Anno Accademico 2014-2015 
This Thesis work was done as part of the research activity of the Istituto di Radioastronomia - Istituto Nazionale di Astrofisica (INAF) in Bologna. 

"To strive, to seek, to find, and not to yield."

Alfred, Lord Tennyson 


\section{Contents}

$\begin{array}{lr}\text { Sommario } & 10\end{array}$

$\begin{array}{ll}\text { Abstract } & 12\end{array}$

1 Introduction $\quad 13$

1.1 Active Galactic Nuclei . . . . . . . . . . . . . . . . . . . . . 13

1.1.1 AGN Paradigm . . . . . . . . . . . . . . . . . . 14

1.2 Radio Loud AGN . . . . . . . . . . . . . . . . . . . . . . . . . . . . . . . 18

1.2 .1 Physical Structure . . . . . . . . . . . . . . . . . . 18

1.3 Radio Galaxies . . . . . . . . . . . . . . . . . . . . . . . . . . . . . . . . 19

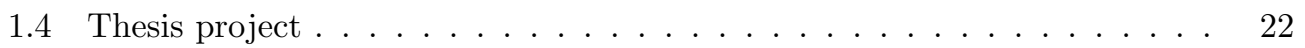

1.4.1 Thesis Outline . . . . . . . . . . . . . . . . 22

2 CSS, GPS and HFP 25

2.1 Synchrotron Spectrum . . . . . . . . . . . . . . . . . 25

2.2 Compact Radio Sources . . . . . . . . . . . . . . . . . . . . . 31

2.3 Classification . . . . . . . . . . . . . . . . . . . . . . . 32

2.4 The Radio Spectra . . . . . . . . . . . . . . . . . . . . . . 33

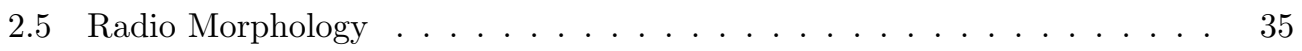

2.6 Polarization . . . . . . . . . . . . . . . . . . . . . . . 36

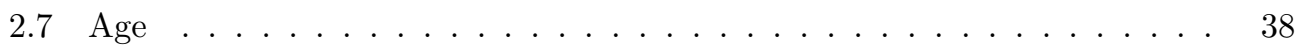

2.7 .1 Radiative Age . . . . . . . . . . . . . . . . . . . 38

2.7 .2 Kinematic Age . . . . . . . . . . . . . . . . . . . . . 39

2.8 The Models . . . . . . . . . . . . . . . . . . . . . . . . . 40

2.8.1 Frustration Scenario . . . . . . . . . . . . . . . . . 40

$2.8 .2 \quad$ Youth Scenario . . . . . . . . . . . . . . . . . 41

3 Radio Observations $\quad 47$

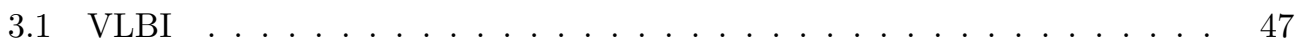

3.1.1 Very Long Baseline Array . . . . . . . . . . . . . . . . . . 48

3.2 The Dataset . . . . . . . . . . . . . . . . . . . . . . . . 51

3.3 Introduction to Calibration . . . . . . . . . . . . . . . . 53

3.4 VLBA data calibration . . . . . . . . . . . . . . . . . . . 54 


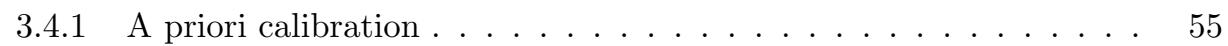

3.4 .2 Fringe fitting $\ldots \ldots \ldots \ldots \ldots \ldots \ldots$

3.4 .3 Bandpass Calibration . . . . . . . . . . . . . . . . . 59

3.4.4 Imaging and self calibration $\ldots \ldots \ldots \ldots \ldots$

4 Data Analysis $\quad 65$

4.1 Multifrequency Images . . . . . . . . . . . . . . . . . . 65

$4.1 .1 \quad \mathrm{~J} 1944+5448 \ldots \ldots \ldots \ldots \ldots \ldots$

$4.1 .2 \quad \mathrm{~J} 0111+3906 \ldots \ldots \ldots \ldots \ldots$

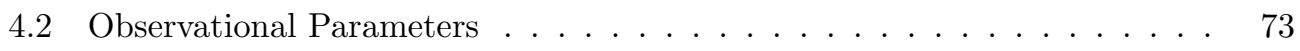

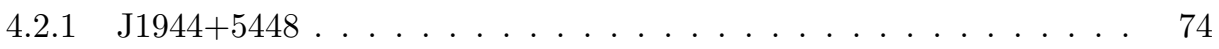

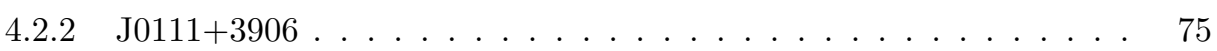

4.3 Morphological Description . . . . . . . . . . . . . . . . 77

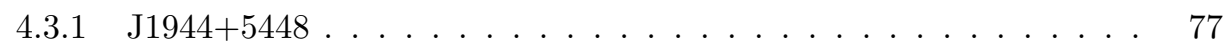

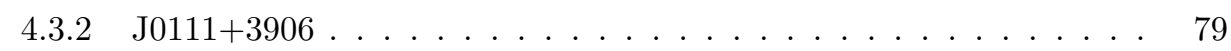

5 Discussion $\quad 81$

5.1 Physical Parameters . . . . . . . . . . . . . . . . . . . . 81

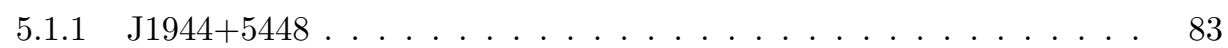

$5.1 .2 \quad \mathrm{~J} 0111+3906 \ldots \ldots \ldots \ldots \ldots \ldots$

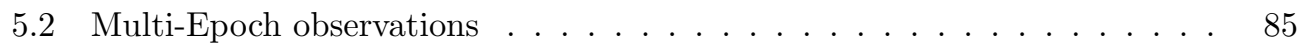

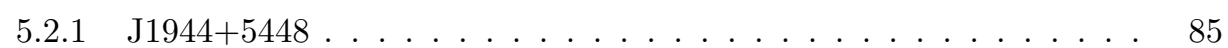

$5.2 .2 \quad \mathrm{~J} 0111+3906 \ldots \ldots \ldots \ldots$

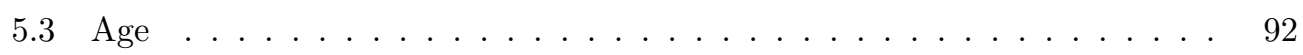

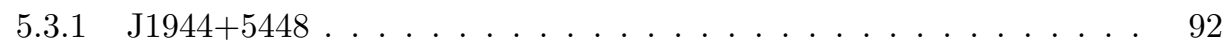

$5.3 .2 \quad \mathrm{~J} 0111+3906 \ldots \ldots \ldots \ldots \ldots$

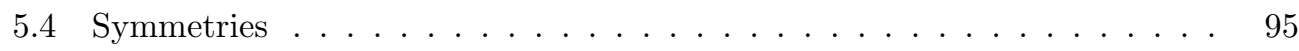

$5.4 .1 \quad \mathrm{~J} 1944+5448 \ldots \ldots \ldots \ldots \ldots \ldots$

$5.4 .2 \quad \mathrm{~J} 0111+3906 \ldots \ldots \ldots \ldots \ldots \ldots$

$5.5 \quad$ Spectral Analysis . . . . . . . . . . . . . . . . . . . . 98

$5.5 .1 \quad \mathrm{~J} 1944+5448 \ldots \ldots \ldots \ldots \ldots$

$5.5 .2 \quad \mathrm{~J} 0111+3906 \ldots \ldots \ldots \ldots \ldots$

$5.6 \quad$ J0111+3906, really young? . . . . . . . . . . . . . . . . . . . . . 114

$\begin{array}{lr}\text { Conclusions } & 120\end{array}$

$\begin{array}{ll}\text { A Physical parameters in radio sources } & 123\end{array}$

$\begin{array}{lr}\text { Bibliography } & 130\end{array}$

$\begin{array}{ll}\text { Acknowledgements } & 131\end{array}$ 


\section{Sommario}

Osservazioni radio ad alta risoluzione con tecniche "Very Long Baseline Interferometry" (VLBI) hanno portato alla scoperta di una sotto-classe di AGN Radio Loud (RL) chiamati "Oggetti Compatti Simmetrici" (CSO). Questi oggetti sono radio galassie estremamente compatte con dimensioni lineari minori generalmente del kpc e molto simili, morfologicamente parlando, alle potenti radio galassie FRII. Un'ulteriore proprietá di questi oggetti compatti riguarda lo spettro radio, che é solitamente convesso. In base alla frequenza di turnover nello spettro radio questi oggetti sono stati classificati come Compact Steep Spectrum (CSS), che hanno un turnover a frequenze minori di un GHz, Gigahertz Peaked Spectrum (GPS), con un turnover intorno al Gigahertz e infine High Frequency Peaker (HFP) che comprende sorgenti il cui turnover sta oltre il Gigahertz. Principalmente esistono due tipi di scenari che spiegano la natura di questi oggetti compatti ma molto simili a radio galassie evolute: il modello della Frustrazione e il modello della Giovinezza. Secondo il primo scenario, le CSO sono sorgenti evolute come le FRII, con etá di circa 10 anni ma la cui espansione attraverso il mezzo inter stellare (ISM) é stata ostacolata dalla presenza di un gas particolarmente denso. La crescita di questi oggetti é stata dunque limitata e la loro evoluzione ed espansione confinata nelle regioni interne delle galassie ospiti. Osservazioni IR e X del mezzo galattico non hanno peró evidenziato nessuna anomalia nella densitá del mezzo e inoltre le galassie ospiti delle CSO sono molto simili a quelle delle FRII, con le stesse proprietá fisiche. Per questo motivo, il modello della frustrazione non viene piú adottato dato che non esiste prova dell'esistenza di un gas cosí denso, capace di confinare la crescita di questi oggetti. Il secondo modello, scenario della giovinezza, invece afferma che le CSO sono compatte perché sono giovani con una etá di circa $10^{3-4}$ anni e non si sono ancora evolute espandendosi nell'ISM. In particolare, le CSO rappresentano la fase "appena nata" di FRII e sono galassie in cui l'attivitá nucleare é stata attivata da poco. Col tempo le CSO si espanderanno prima attraverso il mezzo della galassia ospite e successivamente in quello inter galattico ed evolveranno come FRII.

In questa tesi sono state analizzate le due CSO J1944+5448 e J0111+3906 con l'intento di studiare le loro proprietá morfologiche e spettrali. I dati a disposizione sono relativi a osservazioni multi frequenza in banda L, C e X ottenute con il Very Long Baseline Array (VLBA) nel 2008. I dati sono stati analizzati col software AIPS tramite il quale é stato possibile eseguire le procedure di calibrazione, editing e infine di produzione delle immagini. La sorgente J1944+5448 é stata osservata in banda C e X, ed entrambe le 
immagini prodotte mostrano che é una sorgente con struttura doppia/tripla e asimmetrica caratterizzata da due brillanti hot spot in direzione Est e Ovest, un nucleo centrale e due lobi estesi in direzione Est e Ovest. Anche J0111+3906 é una sorgente doppia/tripla e asimmetrica con due hot spot in direzione Nord Est e Sud Ovest, un nucleo e un getto molto brillante allineato con la hot spot di Nord Est. Questa sorgente é stata analizzata in banda $\mathrm{L}, \mathrm{C}$ e $\mathrm{X}$ e in aggiunta sono stati scaricati dall'archivio i dati in banda $\mathrm{U}$ (da cui sono state ricavate le immagini) realizzati dal progetto MOJAVE. Una volta definita la morfologia e i parametri osservativi, sono stati ricavati i principali parametri fisici di entrambe le sorgenti come la luminositá, l'energia totale e il campo magnetico. Questo permette di arricchire la conoscenza delle principali proprietá di questi oggetti compatti. Osservazioni multi epoca sono state analizzate per determinare la velocitá di separazione delle hot spot e il rate di espansione per queste sorgenti. Un volta nota la velocit con cui queste sorgenti si espandono stato possibile ricavare le etá per entrambe le sorgenti. Per la radio galassia J1944+5448 é stato possibile confrontare l'etá cinematica con quella spettrale e il risultato mostra il buon accordo di questi valori. Per la sorgente J0111+3906 solo l'etá cinematica é stata calcolata. Entrambi i risultati ottenuti relativi all'etá di queste sorgenti mostrano che sono effettivamente oggetti molto giovani con etá di circa $10^{2-3}$ anni, a conferma del modello della giovinezza. Per quanto riguarda le asimmetrie, una volta ottenuti i valori della velocitá delle hot spot rispetto al nucleo centrale, tramite l'assunzione di equilibrio di pressione tra il mezzo esterno e le hot spot si é determinata la densitá del mezzo esterno. Il risultato ottenuto per entrambe le sorgenti rispecchia l'idea che la disomogeneitá del mezzo esterno sia la principale responsabile della forma asimmetrica di questi due oggetti, dal momento che essi sono completamente immersi nel gas della galassia ospite. Infine stato analizzato lo spettro radio della sorgente J0111+3906. Dal momento che lo spettro convesso di questi oggetti si ritiene sia dovuto principalmente ad auto-assorbimento di sincrotrone (SSA), il primo step é stato quello di modellare lo spettro di J0111+3906 con questo meccanismo. Dai risultati, quindi dalla frequenza di turnover e dal flusso di picco ottenuti si é ricavato il campo magnetico e si é confrontato il suo valore con quello di equipartizione precedentemente calcolato. $\mathrm{Si}$ é trovato che i valori erano in buon accordo tra loro. Successivamente lo spettro di J0111+3906 é stato modellato assumendo che il turn over spettrale sia dovuto ad assorbimento libero-libero (FFA), che puó in alcuni casi essere responsabile del profilo, nella regione otticamente spessa, dello spettro radio. I risultati del fit con FFA non sono stati particolarmente buoni e considerando anche il confronto tra i campi magnetici ottenuti utilizzando i parametri del SSA, nel nostro caso favoriamo il modello SSA per la sorgente J0111+3906, come principale responsabile del turnover osservato. Va comunque sottolineato che una combinazione dei due meccanismi potrebbe essere in atto in questa sorgente. 


\section{Abstract}

Multifrequency observations of Radio Loud Active Galactic Nuclei (AGN) discovered a fraction of radio sources which are unresolved on the arc-second scale and with a characteristic convex radio spectrum. According to the frequency of the spectral peak they have been termed Compact Steep Spectrum (CSS), GigaHertz Peaked Spectrum (GPS) or High Frequency Peakers (HFP) radio sources. Given the small angular size, their radio morphology has been determined by means of Very Long Baseline Interferometry (VLBI). It turned out that most objects look like small scale version of the powerful extended (hundred of kpc) Fanaroff-Riley type II (FRII) radio galaxies (O'Dea 1998). In particular those with a clear core detection are known as Compact Symmetric Objects (CSO). Their intrinsically small size is interpreted either in the Frustration Scenario (where the source growth is prevented by an anomalously dense Interstellar Medium of the host galaxy), or, in terms of Youth: the radio galaxy is small because young (Fanti et al., 1995) and it will develop and become larger and larger as it expands and ages. No evidence for a particularly dense medium has been found so far (Siemiginowska et al., 2005). On the other hand estimates of the radiative age (obtained considering the break frequency in the synchrotron spectrum) and the kinematic age (the time taken by the source to expand) of a number of objects confirmed that they are young $\left(\mathrm{t} \leq 10^{3-4} \mathrm{yr}\right.$ ). The analysis of Compact Symmetric Objects is important for understanding the evolution of radio galaxies since they probably represent the early stage of evolved FRII.

In this thesis, two CSO, J1944+543 and J0111+3906, were analyzed with the aim of estimating their expansion rate, their age and their spectral properties. The observations were performed with the Very Long Baseline Interferometry (VLBA) in L, C and X bands. The data calibration and the image production were made using the software package NRAO Astronomical Image Processing Software (AIPS).

The multifrequency images obtained were used to derive the main morphological and physical parameters of these sources. J0111+3906 may be defined as a double/triple source where a central component and two bright hot spot (in the North-East and South West direction) are clearly visible. In this source also a bright jet is observable in the North-West direction. J1944+5448 may be defined a double/triple source as well. In this case the core, two bright hot spots and the two lobes are visible in the West and East directions. J0111+3906 is a very compact radio source with a linear size of about $\sim 40$ pc, while J1944+5448 is more extended ( $\sim 170$ pc). They are both asymmetric sources, 
in particular J1944+5448 which has an arm-length-ratio of about $\sim 2$.

Multi epoch observations were taken into consideration in order to investigate the expansion rate of these source. I estimated the hot spots separation speed of each source and compared the results with those found in the literature. The hot spot advance speed was used to derive the kinematic age of the two CSO. For J1944+5448 I derived also the spectral age making use of the break frequency in the spectrum of the lobes found by Murgia (2003) and using the equipartition magnetic field that I calculated for this source. The kinematic age and the radiative age in J1944+5448 are in very good agreement. Both these radio sources are young $\left(10^{3} \mathrm{yr}\right)$, and in particular J0111+3906 is the youngest. The asymmetric shape of these source may be due to the inhomogeneity of the ambient medium embedding these objects. In order to confirm this hypothesis, an analysis of the density of the ambient medium, by ram pressure and equipartition argument was carry on. This approach confirms the presence of a high density medium that has slowed down the hot spot advance speed and produced the asymmetric shape of these sources. The radio spectrum of J0111+3906 was obtained by multifrequency observations, considering the $\mathrm{L}, \mathrm{C} \mathrm{X}$ bands analyzed in this thesis and the observations in $\mathrm{U}$ band data archive obtained by the MOJAVE project (Lister et al., 2009). The radio spectrum of J0111+3906 was fitted both with a synchrotron self absorption model (SSA) and a free free absorption model (FFA) in order to derive the main mechanism responsible for the spectral turnover. From this comparison we favour that the SSA plays the main role in shaping the radio spectrum of J0111+3906 The analysis of J1944+5448 and J0111+3906 suggest that they are young radio sources embedded in a not homogeneous medium. The results confirm the Youth Scenario. 


\section{Chapter 1}

\section{Introduction}

The aim of this Chapter is to introduce the main features of an Active Galactic Nucleus (AGN) considering the physical model (Section 1.1); particular attention is then reserved to the Radio loud (RL) AGN (Section 1.2). A sub-class of RL AGN, namely the radio galaxies, is then discussed in Section 1.3. An outline of the topics discussed in each Chapter is presented in Section 1.4 where, in addition, the main goals of this thesis work are presented.

\subsection{Active Galactic Nuclei}

Among all types of the extragalactic objects, Active Galactic Nuclei (AGN) are probably the most fascinating and peculiar ones. As a first approximation, AGN is a galaxy where the emission is originated in a powerful central engine and often outshines the emission found in normal (inactive) galaxies, coming from stars and interstellar matter. The high luminosity is produced in a very concentrated volume through physical processes related to the presence of a super massive black hole (SMBH) which is accreting material.

As it is shown in Fig. 1.1 the resultant spectral shape of an AGN is generally complex, extends at various observing bandwidth and it is different from the spectrum of a normal elliptical galaxy (Fg. 1.2), which could be described as a Black Body radiation.

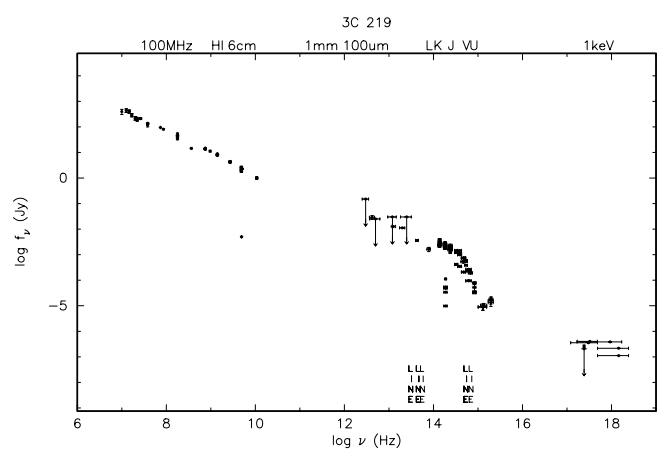

Figure 1.1: Example of the total spectrum of a RL AGN (the source 3c219). 


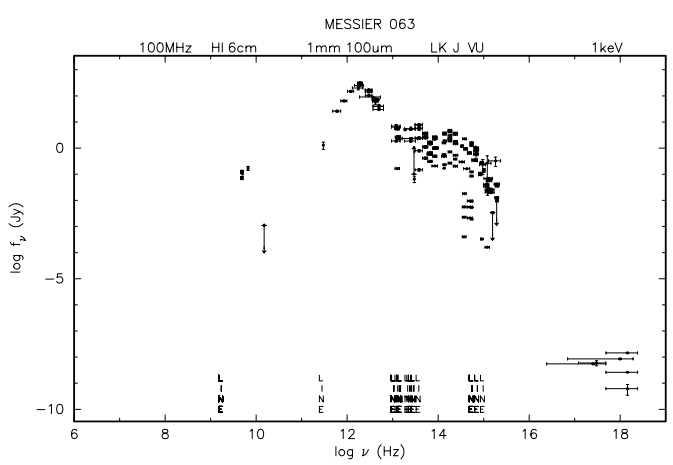

Figure 1.2: Example of the spectrum of a non-active elliptical galaxy (M063), which is mainly black body.

\subsubsection{AGN Paradigm}

The innermost AGN structure has been deeply investigated by multifrequency observations. The commonly accepted intrinsic paradigm which describes the AGN structure is provided in Fig. 1.3, according to which the main AGN components are described as follows:

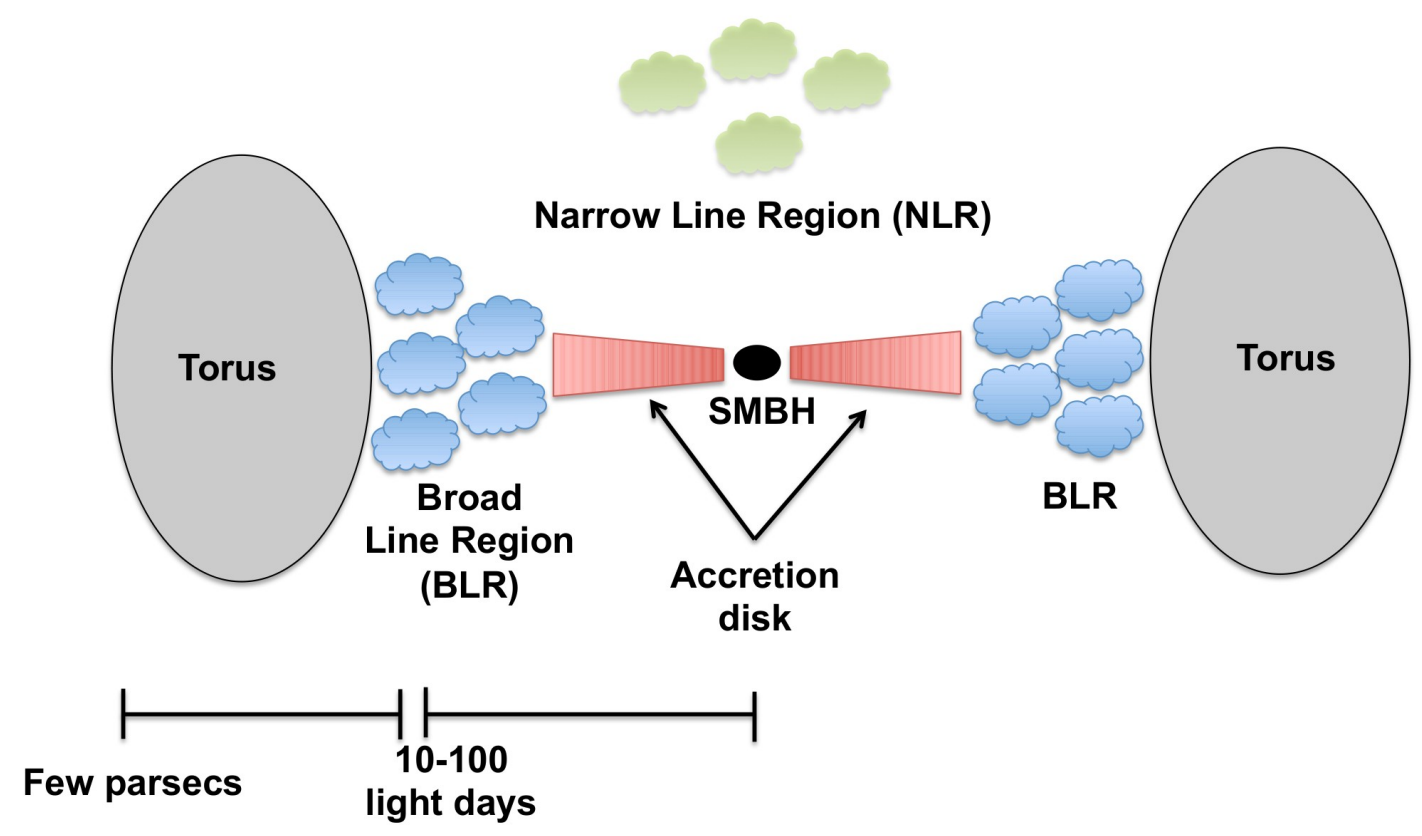

Figure 1.3: Internal structure of an AGN. 


\section{- Central Engine}

Whit this term we refer to the SMBH hosted in the central part of the AGN. Its typical mass is $\mathrm{M}_{\mathrm{BH}} \approx 10^{7-9} \mathrm{M}_{\odot}$. Accretion of gas and dust onto a SMBH is the most efficient method to extract energy from normal matter. This is the foremost way to power the AGN.

\section{- Accretion Disk}

As the gas falling onto the AGN has some residual angular momentum, it will form an accretion disk around the black hole. The simplest model for an accretion disk is that of a geometrically thin and optically thick disk, so that its spectrum can be approximated by a blackbody (Shakura \& Sunyaev, 1973). Thermal emission from this accretion disk is responsible for most of the soft X-ray, UV and optical continuum emission, depending on the mass of the black hole and on the accretion rate. The accretion disk is also the region where some of the outflows are thought to originate.

\section{- Torus}

The emission ranging from the accretion disk, can be obscured by a dust torus extending for few parsec, which surrounds the central region. The torus is also the main supply source for the gas which eventually accretes onto the black hole. The obscuring torus is heated by the incident UV and X-rays radiation from the accretion disk. It has a very high opacity and is visible at IR wavelengths (Krolik \& Pier, 1992).

\section{- Broad Line Region (BLR)}

The BLR lies close to the central engine and is confined inside $1 \mathrm{pc}$. It is composed by high density clouds whose density is about $\mathrm{n}_{\mathrm{e}}^{\mathrm{BLR}} \approx 10^{8}-10^{11} \mathrm{~cm}^{-3}$. Since the BLR's emission lines are produced close to the BH they suffer broadening and Doppler effects. Spectral lines are broadened from a minimum of $\Delta_{\nu} \geq 500 \mathrm{~km} \mathrm{~s}^{-1}$ to $\Delta_{\nu} \geq 10^{4} \mathrm{~km} \mathrm{~s}^{-1}$ (Tthis value is related to the Full Width Half Maximum, FWHM of the lines shape, (Peterson et al., 1993)). The structure of the BLR consists of a central source emitting ionizing radiation roughly spherically, surrounded by distribution of clouds. The side of the cloud facing the central engine is thus highly ionized, while depending on its column density, it results less ionized or mostly neutral on the opposite (back side). Thus we observe the front emitting high-ionization lines from ions such as $\mathrm{He}_{\mathrm{II}}, \mathrm{He}_{\mathrm{I}}, \mathrm{O}_{\mathrm{VI}}, \mathrm{N}_{\mathrm{V}}$, and $\mathrm{C}_{\mathrm{IV}}$, while the back emits low ionization lines from $\mathrm{Mg}_{\mathrm{II}}, \mathrm{Ca}_{\mathrm{II}}, \mathrm{O}_{\mathrm{I}}$, and $\mathrm{Fe}_{\mathrm{II}}$. These are some examples of broad ionized lines of an AGN, but we can find also broad neutral lines such as $\mathrm{H}_{\alpha}$ and $\mathrm{H}_{\beta}$. The BLR is also unresolved even in the nearest AGN, so we do not have enough information about its structure. The emission of the central part of the AGN has a direct influence on the emission line of the BLR. Indeed, flux variations of the central engine cause variations on the broad line emission. Thus, the latter 
are delayed by the time spent by the light to travel from the central part to the BLR. The knowledge of the time delay and of the gas kinematics allows us to estimate the Black Hole mass with an accuracy of about a factor of three, (Peterson, 2006). This method is called Reverberation Mapping (Peterson, 1993).

\section{- Narrow Line Region (NLR)}

The Narrow Line region marks the "borderline" region of direct influence of the AGN. The NLR probably extends for $10^{2-3}$ pc, partially outside the torus. It is optically resolved and is formed by clouds with an electron density $n_{\mathrm{e}}^{\mathrm{NLR}} \approx$ $10^{2}-10^{4} \mathrm{~cm}^{-3}$ that is lower than that found in the BLR. Spectral lines from the NLR do not shown variability, reflecting the fact that they come from a more extended region than the BLR. [OIII] and [SII] show narrow emission lines. Finally the most particular feature of the NLR regards the so called 'Ionization cones'. These are regions confined by the collimation of light from the nuclear source. Inside these cones the excitation conditions are roughly uniform but drop very rapidly outside (Antonucci, 1993). The commonly accepted idea is that the ionizing radiation coming from the central region has an axial symmetric distribution. This causes the cone-shaped structure of the ionized region of gas cloud that we observe. This last aspect was one of the major evidence of the preferential emission direction of the AGN, and gives support to the idea of an anisotropic emission from these particular objects.

\section{The Unified Model}

As discussed above, one of the key aspects of AGN is their anisotropic radiation, which derives from an anisotropic structure (Fig.1.3). This condition allows us to explain the different observational properties for the various zoology of AGN, under the assumption of an unique physical model: the Unified Model.

This geometrical model explains the differences among Type 1 AGN (those with broad lines in the optical spectrum, and substantial UV and X-ray radiation) and Type 2 AGN (those with only narrow lines and little/no UV-Xrays) based on the line of sight (l.o.s.). In the latter the l.o.s crosses the molecular dusty torus which is optically thick to high energy radiation.

An illustration of this model is shown in Fig. 1.4 where in addition AGN are divided in two main classes depending on the radio and optical power. Based on the ratio between the emission at $5 \mathrm{GHz}$ (radio) and the B-band (optical), AGN are classified as either Radio Loud (RL) or Radio Quiet (RQ) although this does not mean that they are radio silent. In fact, most of the more spectacular nearby radio sources (e.g. the Fanaroff-Riley type I) would be classified as RQ despite being rather big and bright radio sources.

In the framework of this thesis we will focus our attention only on Radio Loud AGN, which we are going to describe in the following Section. 


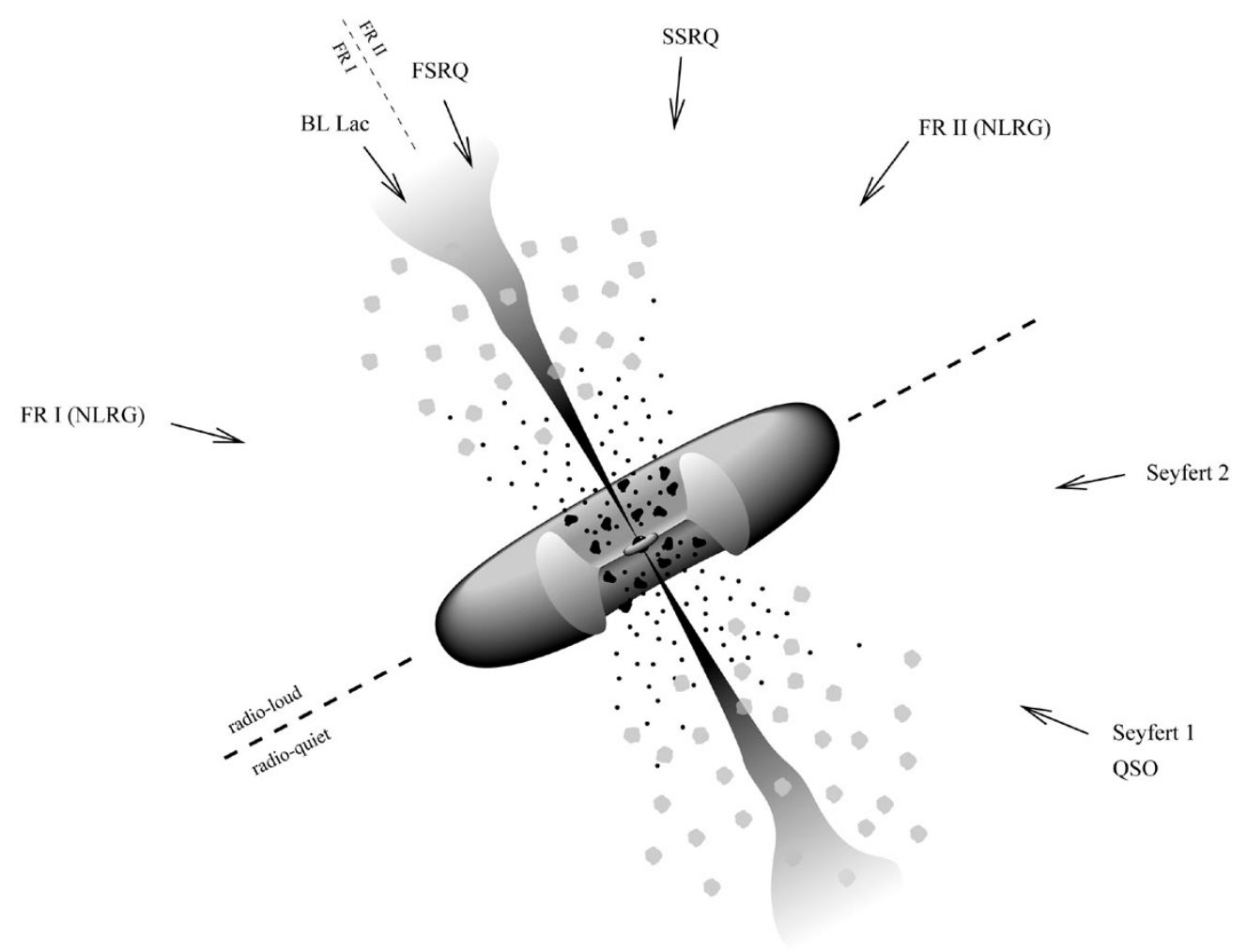

Figure 1.4: AGN Unified Model (Urry \& Padovani, 1995). In this cartoon the main classes of radio loud and radio quiet AGN are shown. FRI(NLRG) and FRII (NLRG): Fanaroff-Riley tipe I and II (Narrow Line radio galaxy), FSRQ: Flat spectrum radio quasar, SSRQ: steep spectrum radio quasar. 


\subsection{Radio Loud AGN}

Radio Loud (RL) AGN represent a small fraction of all the AGN zoology. Roughly 15$20 \%$ of the observed AGN are Radio Loud (Urry \& Padovani, 1995). The radio spectra of RL AGN is non thermal and is mostly due to the synchrotron emission and inverse compton of relativistic electrons.

The most characteristic feature in RL AGN is the presence of a bipolar outflow of relativistic plasma from the innermost regions which gives origin to two symmetric jets observed on scales ranging from the parsec up to the Mega-parsec.

\subsubsection{Physical Structure}

The physical structure of a RL AGN can be described by four main components, even if not all these components are present in all RL AGN. In addition, we have to take into account that the aspect of a source may depend on the angular resolution and the frequency of the observation. Considering Fig.1.5, the fundamental elements of the RL AGN structure are the following:

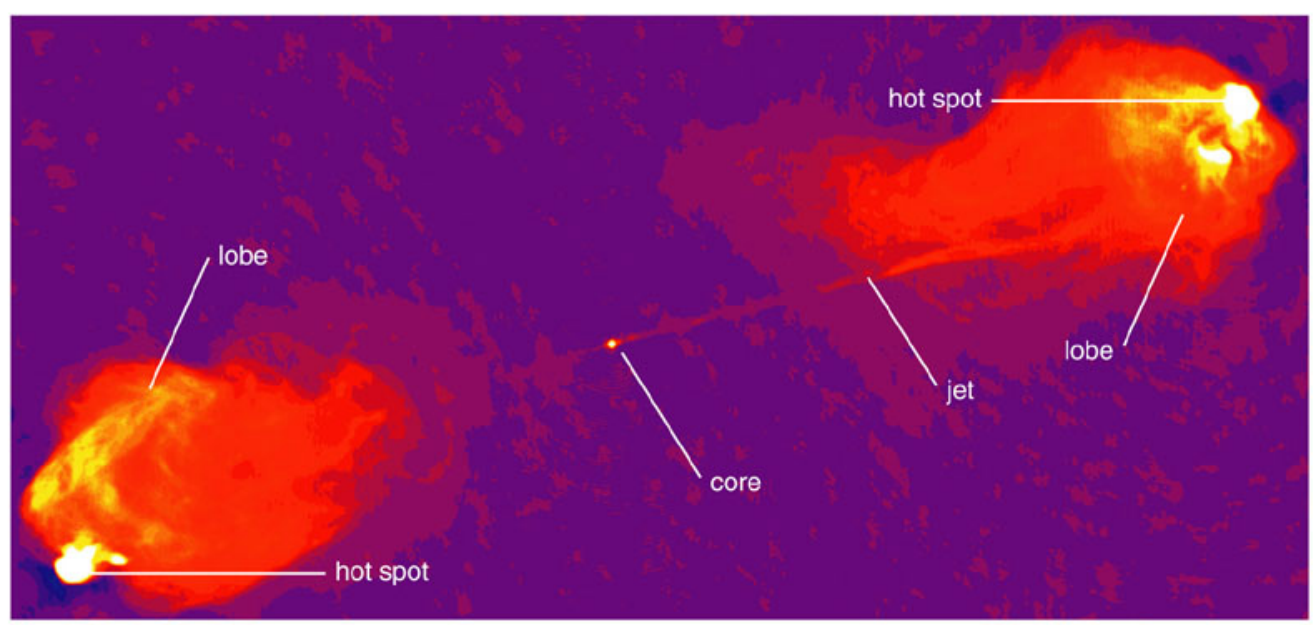

Figure 1.5: The different components of the RL AGN structure.

- JETS:

The jet is a collimated structure, often with a knotty aspect, where relativistic particles and magnetic field ejected from the central region flow towards the surrounding medium. The spectral index is typically about $\alpha \approx-0.7$ (optically thin).

\section{- CORE:}

It is the central compact (sub-pc scale) component hosted in the central region of the optical host galaxy. It is interpreted as the place of origin of whole energy output of the source. The radio emission of the core is the closest to the SMBH. In addition, 
the core is the base of the jet in the transitional region between the optically thin and thick regime. Because its compact size the core spectrum is flat/inverted.

- LOBES:

They appear as large diffuse structures, symmetrically located with respect to the central core. They extend over tens of pc (Compact radio galaxies) up to Mpc (giant radio sources). Lobes usually show a low surface brightness compared to the other RL AGN components. Their radio spectra are steep with a spectral index $(\alpha \leq-1)$, indicating the presence of old electron populations that are ageing in these structures without any re-acceleration (Murgia et al., 1999).

- HOT SPOTS:

In the powerful radio sources (see Section 1.3) as the jet collides with the surrounding gas it drives a shock and creates the hot spots which appear as bright and compact regions located at the edge of the sources. They are usually well aligned with the central core. Hot spots have a typical power-law spectrum with spectral index $\alpha \sim-0.5, \sim-0.8$ indicating the presence of particle re-acceleration.

\subsection{Radio Galaxies}

Radio galaxies represent an interesting sub-class of Radio Loud AGN. Taking into account their radio power and the physical components described in the previous section, radio galaxies are further divided into two sub-classes: Fanaroff-Riley type I (FRI) and type II (FRII) as proposed by Fanaroff \& Riley (Fanaroff \& Riley, 1974). Classical FRI and FRII typically extend from a tens of $\mathrm{kpc}$ up to $\approx \mathrm{Mpc}$ and their age is $\mathrm{t}_{\mathrm{Age}} \approx 10^{7} \mathrm{yr}$. In order to point out the morphological difference between these two type of RL AGN, examples of FRI and FRII are shown in Fig. 1.6

- FRII: They show high radio power with $\mathrm{P}_{1.4 \mathrm{Ghz}} \geq 10^{24.5} \mathrm{~W} \mathrm{~Hz}^{-1}$. Their lobes are usually extended and with an elongated shape. They show bright and compact hot spots, so their luminosity increases towards the edge of the source. For this reason FRII are also called Edge Brightened radio galaxies (Fig.1.6 bottom). In the optical they are associated with either a galaxy or a quasar.

- FRI: They are radio sources with $\mathrm{P}_{1.4 \mathrm{Ghz}} \leq 10^{24.5} \mathrm{~W} \mathrm{~Hz}^{-1}$. They show usually two-sided jets, often symmetric, well visible and extremely bright. Usually FRI do not show hot spots, so their luminosity is more concentrated near the central region, and, for this reason, they are also called Edge Darkened radio galaxy. (Fig.1.6 top) 


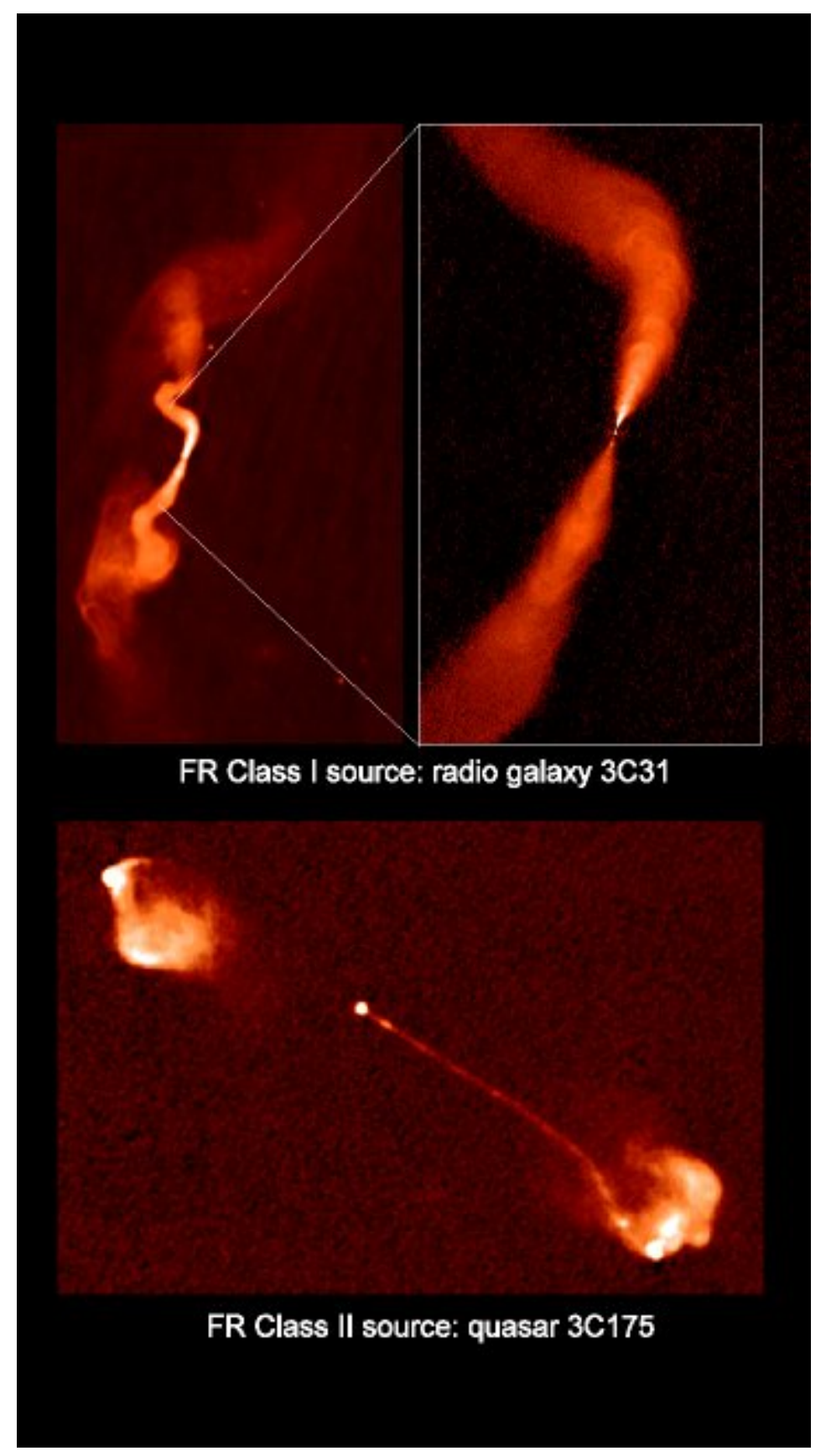

Figure 1.6: Two examples of RL AGN: a FRI (top) and a FRII (bottom). 
FRII usually show one-sided jet that is often weaker with respect to those of the FRI. Moreover the jet of FRII is relativistic with respect to the FRI. The difference between the jet in FRI and FRII may be connected with a different energetic efficiency of the jet itself. This is strictly related to the BH efficiency of converting mass into energy (Owen \& Ledlow, 1994). Either the particular ambient medium conditions with which the jets interact, or their initial energy power, or a combination of both, may produce observable differences between the jets of FRII and FRI (Meliani et al., 2010).

The classification of FRII and FRI mentioned above, is completely independent of the source linear size. Alternatively, if we classify radio galaxies depending only on their linear dimension, we should find a sub-class of objects which extends on sub-galactic scales while sharing the same properties of the large extended radio galaxies.

These sub-class of radio sources includes compact radio loud AGN which will be the main topic of this thesis work.

Compact radio loud AGN will be investigated in more details on Chapter 2. 


\subsection{Thesis project}

This thesis project focuses on the study of two compact radio loud AGN by using multifrequency observations obtained with the VLBA. I will investigate the radio properties of these sources, estimate their kinematic age and, when possible, the spectral age. By multifrequency observations is possible to obtain the radio spectra of each component of these sources. In addition the analysis of the hot spot separation speed and the morphological features could help in order to investigate the ISM conditions. These pieces of information are important for improving our knowledge in their evolution within the host galaxy.

\subsubsection{Thesis Outline}

- Chapter 2 provides a detailed description of the main features of compact radio sources considering their radiative and morphological characteristics. In addition I explain the methods to compute the age of radio sources and I describe their morphology and their polarization properties. Finally I present the two models that try to explain the nature of these compact sources.

- Chapter 3 I give a description of the instrument used in this work, the VLBA, focusing on its main properties. Then I present the data sets used in this thesis work. The data underwent a series of process of calibration and reduction which is described in detail.

- Chapter 4 I present the multifrequency images obtained for each source. Then I show the main observational parameters estimated from the images of these compact sources. Then I describe the physical and morphological features of these source on the basis of the images and the parameters obtained.

- Chapter 5 I describe the physical parameters calculated for each source component such as the luminosity, the energy and the magnetic field. Then I describe the multi epoch observations analysis and discuss the results obtained from this approach. 
The symmetries in the morphological structure of the two sources is discussed. Finally a detailed spectral analysis of one of the two radio galaxies is discussed with a comparison between two physical models.

Throughout all this thesis we assume $\mathrm{H}_{0}=71 \mathrm{~km} \mathrm{~s}^{-1} \mathrm{Mpc}^{-1}, \Omega_{\mathrm{M}}=0.27$ and $\Omega_{\lambda}=0.73$ in a flat Universe. 


\section{Chapter 2}

\section{CSS, GPS and HFP radio sources}

In this Chapter the key properties of intrinsically compact RL AGN are described. Section 2.1 gives a description of the properties of the synchrotron mechanism which is responsible for the radio emission of extragalactic radio sources. The general properties of compact radio loud AGN are described in Section 2.2. and Section 2.3 that gives a classification of these sources. Then the spectral and morphological features of compact objects are discussed (Section 2.4 and 2.5). The polarization features are described in Section 2.6. The method used to estimate the age are presented in Section 2.7. The models that explain the nature of these compact radio sources are presented in Section 2.8.

\subsection{Synchrotron Spectrum}

Synchrotron radiation is due to the acceleration of a population of relativistic particles in a magnetic field. The energy particle distribution is described by a simple power-law as:

$$
\mathrm{N}(\mathrm{E})=\mathrm{N}_{0} \mathrm{E}^{-\delta}
$$

$\mathrm{N}(\mathrm{E})$ is the number of particles with energy $\mathrm{E}, \delta$ is the index of the power law and $\mathrm{N}_{0}$ is a constant. An electron population with this energy distribution produces a radio emission that is again a power law. The flux density $\mathrm{S}$ is directly related to the frequency $\nu$ according to:

$$
\mathrm{S}(\nu) \propto \nu^{\alpha}
$$

In Eq.2.2, $\alpha$ is the Spectral Index defined as

$$
\alpha=-\frac{\delta-1}{2}
$$




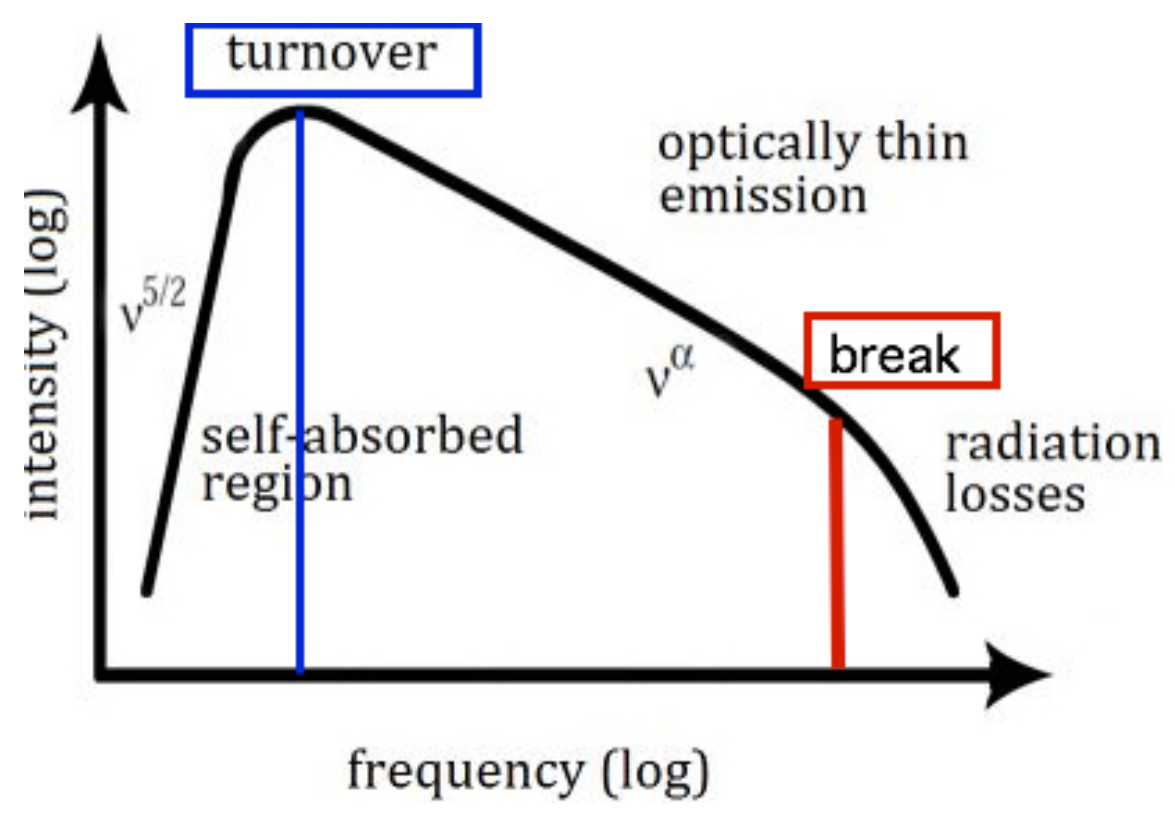

Figure 2.1: Theoretical Synchrotron Spectrum

The radio spectra of radio sources usually are well represented by power-laws (Eq. 2.2 ), even if their spectra deviate from the theoretical one. In fact absorption becomes important when the photons transfer a large amount of their energy to the electrons. In this case the radio source becomes opaque to its own radiation. This process is called Synchrotron Self Absorption or SSA (see Fig. 2.1). It is related to the fact that the brightness temperature $\mathrm{T}_{\mathrm{e}}$ of the source becomes equal to the kinetic temperature $\mathrm{T}_{\mathrm{k}}$ of the electrons. In particular the kinetic temperature is:

$$
\mathrm{T}_{\mathrm{k}} \approx \frac{\mathrm{E}}{\mathrm{k}} \propto \mathrm{H}_{\perp}^{-1 / 2} \nu^{1 / 2}
$$

where $\mathrm{E}$ is the energy of the electrons, $\mathrm{H}_{\perp}$ is the projection of the magnetic field along the velocity direction of the electrons and $\mathrm{k}$ is the Boltzmann constant. The brightness temperature is:

$$
\mathrm{T}_{\mathrm{e}}=\frac{1}{2 \mathrm{k}} \mathrm{B}(\nu) \frac{\mathrm{c}^{2}}{\nu^{3}}
$$

where $\mathrm{B}(\nu)$ is the synchrotron brightness, $\mathrm{c}$ is the speed of light. $\mathrm{B}(\nu)$ depends on the optical depth $\tau$ as follows: 


$$
\mathrm{B}(\nu) \propto \mathrm{H}_{\perp}^{-1 / 2} \nu^{5 / 2} \quad \text { for } \quad \tau \gg 1
$$

in the optically thick regime, and

$$
\mathrm{B}(\nu) \propto \nu^{\alpha} \quad \text { for } \quad \tau \ll 1
$$

in the optically thin region. $\mathrm{B}(\nu)$ is related to the flux density $\mathrm{S}(\nu)$ by $\mathrm{B}(\nu)=\mathrm{S}(\nu) / \Delta \Omega$ where $\Delta \Omega$ is the solid angle under which the source is observed.

Therefore at low frequencies the flux density $\mathrm{S}$ increases with a spectral index $\propto \nu^{2.5}$, while in the optically thin part it decreases with $\mathrm{S} \propto \nu^{\alpha}$. However this is only a theoretical model in which the radio source are homogeneous components. However, radio sources consist of many sub-structures as jets, hot spots and lobes (Section 1.3), each of them with its own spectral properties. Therefore the observed overall spectrum is the superposition of the spectra of all the radio sources components, and so it does not match the theoretical $\alpha=2.5$ slope (O'Dea, 1998).

As shown in Fig.2.1 the radio spectrum of the synchrotron emission can be divided into three regimes. At low frequencies, where the source is opaque, the spectrum rises as $\nu^{2.5}$ due to the self-absorption of the radiation, up to a peak frequency $\nu_{\text {to }}$ which is called turnover frequency, while at higher frequencies, in the optically thin region, the spectrum shows the classical power law. At even higher frequencies we usually find a break in the spectrum which is related to the rate of energy losses suffered by the electron population and to a continuos injection of new particles.

The main radiative processes causing energy losses to relativistic electrons are Synchrotron and Inverse Compton emission.

Inverse Compton (I.C) and Synchrotron (S.) losses can be described as follows:

$$
\begin{aligned}
& -\left(\frac{\mathrm{dE}}{\mathrm{dt}}\right)_{\mathrm{S} .}=\frac{4}{3} \sigma_{\mathrm{T}} \gamma^{2} \mathrm{c} \frac{\mathrm{H}^{2}}{8 \pi} \\
& -\left(\frac{\mathrm{dE}}{\mathrm{dt}}\right)_{\text {I.C. }}=\frac{4}{3} \sigma_{\mathrm{T}} \gamma^{2} \mathrm{c} \mathrm{u} \mathrm{f}_{\mathrm{f}}
\end{aligned}
$$

where $\sigma_{\mathrm{T}}$ is the Thomson cross section, $\gamma$ is the Lorentz factor of the electrons, $u_{f}$ is the mean energy density of the radiation field, $\mathrm{H}$ the magnetic field and $\mathrm{c}$ the speed of light.

Then, combining Eq. 2.7 and 2.8 we obtain:

$$
-\left(\frac{\mathrm{dE}}{\mathrm{dt}}\right)_{\mathrm{S}+\mathrm{I} . \mathrm{C} .}=\mathrm{b} \mathrm{E}^{2}\left(\mathrm{H}_{\perp}^{2}+8 \pi \mathrm{u}_{\mathrm{f}}\right)
$$

where $\mathrm{b}$ is a constant and $\mathrm{E}$ is the electron energy $\left(\mathrm{E}=\gamma \mathrm{m} \mathrm{c}^{2}\right.$ where $\mathrm{m}$ is the mass 
particle). Both IC and $\mathrm{S}$ losses depend on $\mathrm{E}^{2}$ so the most energetic particles suffer the highest energetic losses.

Integrating Eq. 2.9 we obtain:

$$
\mathrm{E}(\mathrm{t})=\frac{\mathrm{E}_{0}}{1+\mathrm{E}_{0} / \mathrm{E}^{*}}
$$

where $\mathrm{t}$ is the time, $\mathrm{E}_{0}$ is the energy of particles at $\mathrm{t}=0$ and $\mathrm{E}^{*}$ is the energy break defined as:

$$
\mathrm{E}^{*}=\frac{1}{\mathrm{~b}(\mathrm{H} \sin \theta)^{2} \mathrm{t}}
$$

where $\theta$ is the pitch angle defined as the angle between the magnetic field and the electron velocity direction.

Particles with smaller $\theta$ have larger $\mathrm{E}^{*}$. In the case of a re-isotropization of the pitch angle (e.g. Jaffe \& Perola, 1973) the energy break is

$$
\mathrm{E}_{\mathrm{JP}}^{*}=\frac{1.5}{\mathrm{~b} \mathrm{H}^{2} \mathrm{t}}
$$

As Eq. 2.12 shows, the energy does not depend on $\theta$ so electrons with the same energy age similarly.

At any given epoch $t$ corresponds an energy break $\mathrm{E}^{*}$ below which the initial spectral energy is substantially unchanged, while, for $\mathrm{E}>\mathrm{E}^{*}$ the spectrum is modified. Similarly to the break energy we define a break frequency $\nu^{*}$. As the electron population ages, the break frequency moves progressively to lower frequencies. For this reason, old relativistic electron populations show spectra with a break frequency lower than that of young objects.

Therefore, if the magnetic field is known it is possible to calculate the Radiative Age of the source, from its break frequency (Murgia et al., 1999):

$$
\nu^{*} \propto\left(\mathrm{E}^{*}\right)^{2} \mathrm{H}
$$

and

$$
\nu^{*} \propto \mathrm{t}^{-2} \mathrm{H}^{-3}
$$

the value of the radiative age is ${ }^{1}$ :

$$
\mathrm{t}_{\mathrm{rad}}=5.03 \times 10^{4} \cdot \mathrm{H}^{-1.5}\left[(1+\mathrm{z}) \cdot \nu^{*}\right]^{-0.5} \quad \mathrm{yr}
$$

where $\mathrm{z}$ is the redshift, the magnetic field $\mathrm{H}$ is expressed in milligauss $(\mathrm{mG})$ and the frequency $\nu$ in GHz.

\footnotetext{
${ }^{1}$ In this case Inverse Compton losses are neglected and the magnetic field $\mathrm{H}$ is supposed to be constant (Murgia 2003).
} 
To allow an appropriate understanding of the radio spectrum features, another important situation should be considered.

Since the emission from an electron population with an initial power law energy distribution shows a steepening for $\mathrm{E}>\mathrm{E}^{*}$, we must take into account two important boundary situations regarding the origin of these relativistic particles:

- single injection of relativistic particles.

- continuous particles injection.

The evolution of a relativistic electron population can be described following three main paths, as summarized in (Murgia, 2003) and (Nagai et al., 2006) :

- KP model, by Kardashev \& Pacholczyk (Pacholczyk, 1970). It assumes a single pitch angle $\theta$ for all the electrons with respect to the magnetic field lines which is preserved with time and a single burst of particle injection. According to KP model the electron density and the magnetic field are assumed homogeneous along the source depth.

Thus the break $\nu^{*}$ can be expressed as:

$$
\frac{\nu^{*}}{(\mathrm{GHz})}=1.12 \times 10^{9}\left(\frac{\mathrm{H}}{\mathrm{mG}}\right)^{-3}\left(\frac{\mathrm{t}}{\mathrm{yr}}\right)^{-2}
$$

The energy break in this case is the same as in Eq. 2.11.

This produces a tail of high energy particles in the energy spectrum which results "flatter" with respect to the JP.

- JP model, by Jaffe and Perola (Jaffe \& Perola, 1973). Differently from the KP model, the JP model assumes a continuos re-isotropization of the pitch angle of the relativistic particles. (The timescale for the continuos re-isotropization is assumed to be much shorter than the radiative lifetime so $\left.(\mathrm{H} \sin \theta)^{2}=\left(2 / 3 \mathrm{H}^{2}\right)\right)$. In this case the break is:

$$
\frac{\nu_{\mathrm{b}}}{(\mathrm{GHz})}=2.52 \times 10^{9}\left(\frac{\mathrm{H}}{\mathrm{mG}}\right)^{-3}\left(\frac{\mathrm{t}}{\mathrm{yr}}\right)^{-2}
$$

According to this model the energy does not depend on $\theta$ as shown by Eq. 2.11. This results in a sharp energy cutoff in the energy electron distribution, with a synchrotron spectrum showing an exponential drop at high frequencies. For this reason the spectrum results steeper than in the case of KP.

In both cases $\nu^{*}$ move to lower frequencies with time. 
- CI model, from Kardashev (Kardashev, 1962). In this case the sources are expected to be continuously replenished by a constant flow of fresh relativistic particles. The energy distribution of these particles is well represented by : $\nu^{\alpha+0.5}$ instead of an exponential drop as in the JP model.

Thus the CI model can be adopted for regions where it is hard to distinguish aged electrons from newly injected electrons. In particular an ensemble of different populations (e.g. the lobes and the hot spots) can be well fitted by the CI model. However, if we are considering resolved sources, the KP and JP models are adequate for fitting the lobes. In particular, the spectra of the hot-spots are well fitted by models predicting a continuous injection of fresh particles (CI) (Murgia, 2003), while lobes and extended structures are better fitted by single-injection models (JP and KP).

An example of the spectra of the lobes and the hot spots in two radio sources is shown in Fig. 2.2
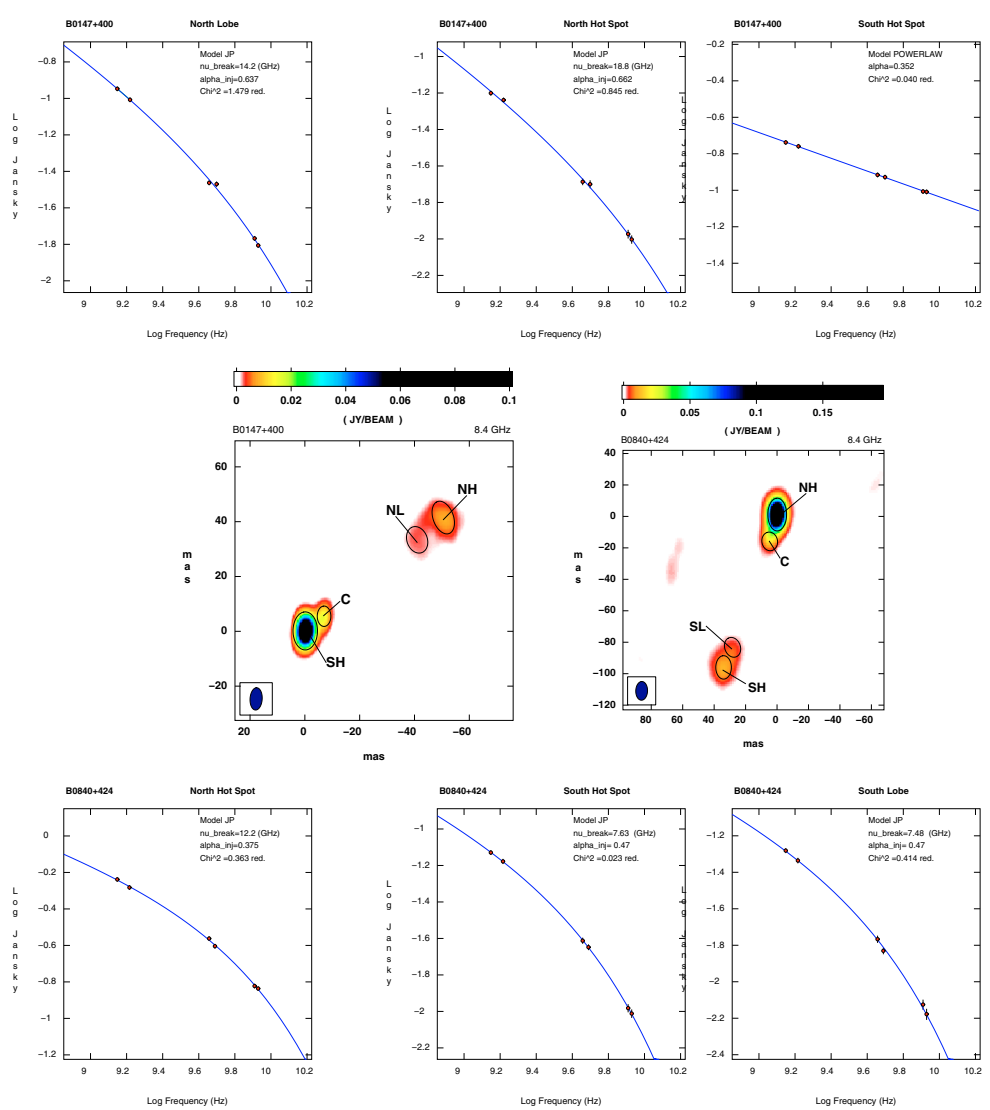

Figure 2.2: The local spectra in the lobes, and in the hot-spots of the sources B0147+400 and B0840+424 (Orienti et al., 2007). 


\subsection{Compact Radio Sources}

This class of objects includes either compact radio galaxies and compact radio quasars. They are luminous $\left(\mathrm{L}_{178 \mathrm{MHz}} \geq 10^{26} \mathrm{~W} \mathrm{~Hz}^{-1}\right.$ ) radio sources, associated with an elliptical galaxy. Extragalactic radio sources belonging to this class are quite similar to FRII but they have different linear size (LS).

A morphological comparison between a compact galaxy and an extended FRII is shown in Fig. 2.3: on top panel we can see the FRII Cygnus A, extended for about $100 \mathrm{kpc}$, while the bottom panel shows the compact radio galaxy J1944+5448, extended for only a hundred of pc. The key component are about the same although some marginal differences are related to the different physical conditions of the ambient medium surrounding the relativistic plasma.
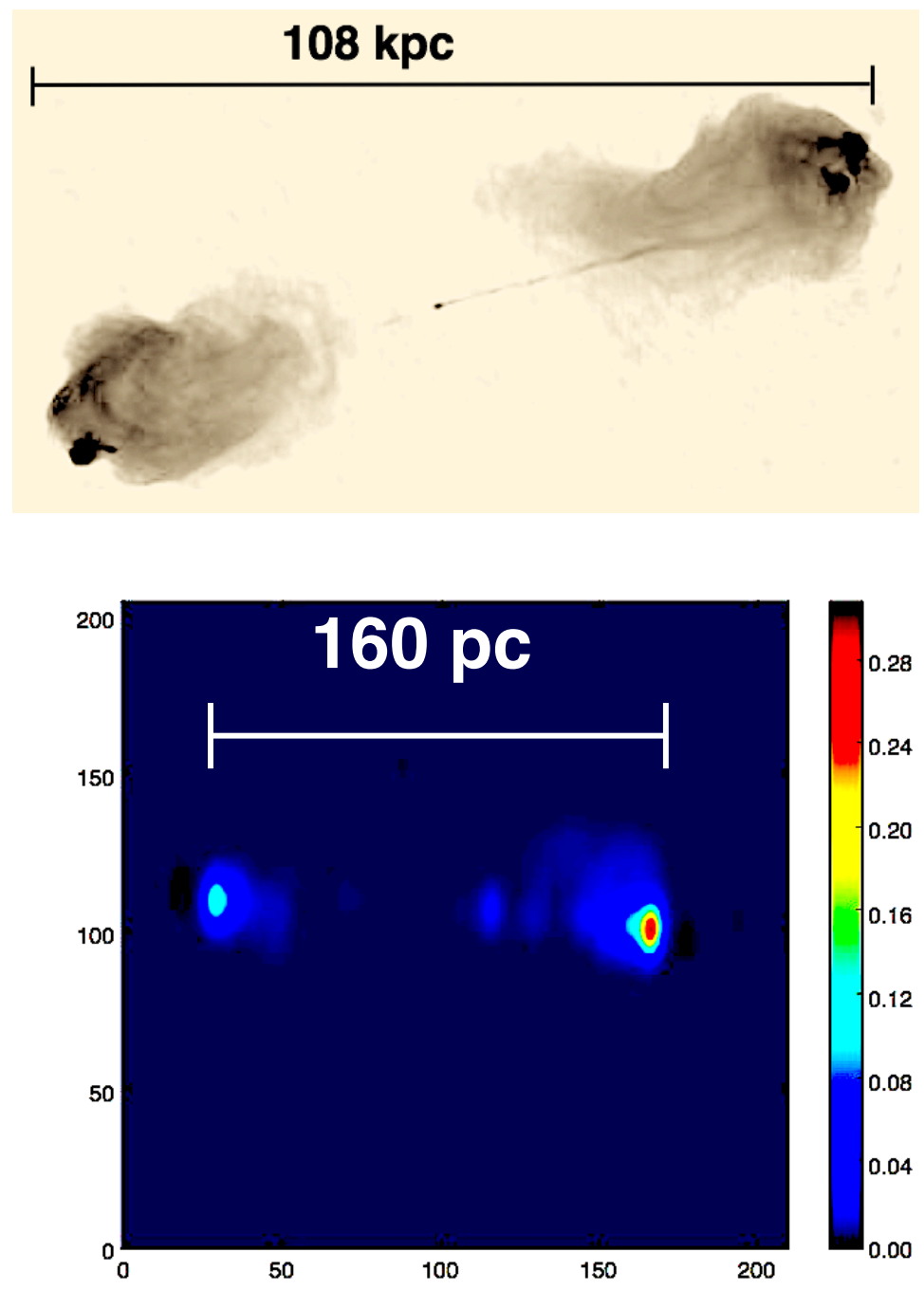

Figure 2.3: Classical FR II radio source (top) and a Compact Symmetric Object (bottom.) 
Compact radio sources can be simply described as a scaled-down version (with physical size from 0.01 to $\sim 15 \mathrm{kpc}$ ) of the powerful, edge-brightened FRII (Snellen et al., 1998). In addition classical extend radio loud AGN, exceed by many times the size of the host elliptical galaxy. They interact mainly with the Inter Galactic Medium (IGM) or Intra Cluster Medium (ICM) if they belong to a cluster. On the contrary, compact sources result completely embedded in the host galaxy and interact only with its InterStellar Medium (ISM). This difference about the ambient medium with which the relativistic plasma interacts, is a fundamental key to understand the growth and the evolution of the radio source. In fact, dense clouds could impact with the jets and slow down the source expansion. (This aspect will be discussed in more detail in Section 2.5).

\subsection{Classification}

In the following, I classify Compact Radio Loud AGN depending only on their spectral features derived from their radio spectrum. Three main spectral classes of compact radio sources whose emission is fully within the ISM of the host galaxy are defined as follows:

- Compact Steep Spectrum radio sources (CSS) have the turnover frequencies between $\approx 100-500 \mathrm{MHz}$.

- Gigahertz Peaked Spectrum (GPS) radio sources have the spectral peak around 1 GHz.

- High Frequencies Peakers (HFP) have the spectral peaks at a few $\mathrm{GHz}(\geq 5-10 \mathrm{GHz})$.

The above classifications are the historical definitions of CSS and GPS (Fanti et al., 1990) and HFP (Dallacasa et al., 2000). As shown in Fig.2.4 the different turn over frequencies are a key property of these three classes of compact sources, although there is not a clear cut in this classification.

Thanks to the high spatial resolution provided by interferometric technique, it has been found that CSS have size between 1 and $20 \mathrm{kpc}$. They interact mainly with the ISM of the host galaxy. GPS have size lower than $1 \mathrm{kpc}$, while HFP usually extend one order of magnitude less than the GPS (Dallacasa et al., 2000). For this reason both GPS and HFP result completely embedded in the innermost part of the host galaxy, interacting mainly with the NLR.

In addition a morphological classification was proposed by Readhead et al. (1995). In this case the source size was taken into account as a tool to distinguish among the various type of radio sources. In particular it has been used in order to classify sources considering only two-sided objects in which two lobes of emission straddling the center of activity are clearly visible (Readhead, 1995). 


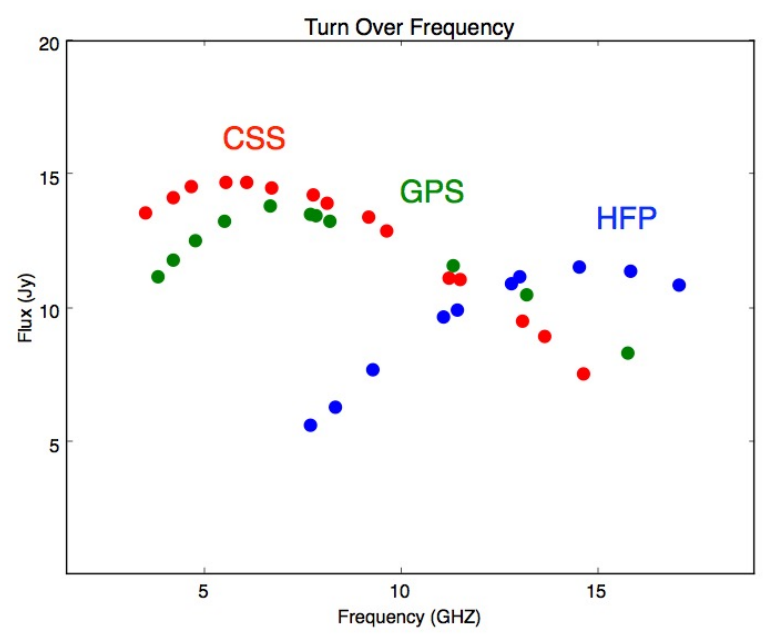

Figure 2.4: Different spectra of each class of compact radio sources (Dallacasa et al., 2000).

Readhead et al. (1995) defined Compact Symmetric Object (CSO), those sources with the lobe separations less than $1 \mathrm{kpc}$ and, similarly, Medium Symmetric Object (MSO), those with the lobe separation in the range $1-15 \mathrm{kpc}$. As a consequence of this classification the most elongated radio sources as FR II or FR I, whose lobe separation is more than $15 \mathrm{kpc}$ (i.e. outside the host galaxy) are called Large Symmetric Object (LSO). According to this classification, GPS and HFP are manly included in the CSO, while CSS belong to the MSO sources, even if a strict application of this definition is difficult. It is worth noting that this classification is purely arbitrary without a physical meaning, but it can be useful in order to distinguish between the various type of sources.

\subsection{The Radio Spectra}

The radio spectra of CSS, GPS and HFP usually are well represented by power-laws, even if their spectra deviate from the theoretical one. In fact, as mentioned in Section 2.1 a fundamental ingredient which usually influences the spectral shape of compact radio sources is the SSA. Altough there are some cases in which also Free Free absorption plays a role.

However observations suggest that the SSA is the most likely mechanism responsible for the observed spectral shape and turnover frequencies (Snellen et al., 2000b). In addition to support this hypothesis, O'Dea et al. (1997) found a simple relation between the turn over frequency $\nu_{\text {t.o. }}$ and the projected linear size, LS, of the radio sources, defining a plane $\nu_{\text {t.o. }}-$ LS through which sources should evolve as:

$$
\nu_{\text {t.o. }} \propto \mathrm{LS}^{-0.65}
$$


The connection between the turnover frequency and the linear size suggests that the mechanism responsible for the $\nu_{\text {t.o. }}$ is strictly connected with the source size (O'Dea \& Baum, 1997). For this reason SSA seems to be the most probable mechanism, if we assume a self similar growth of the sources.

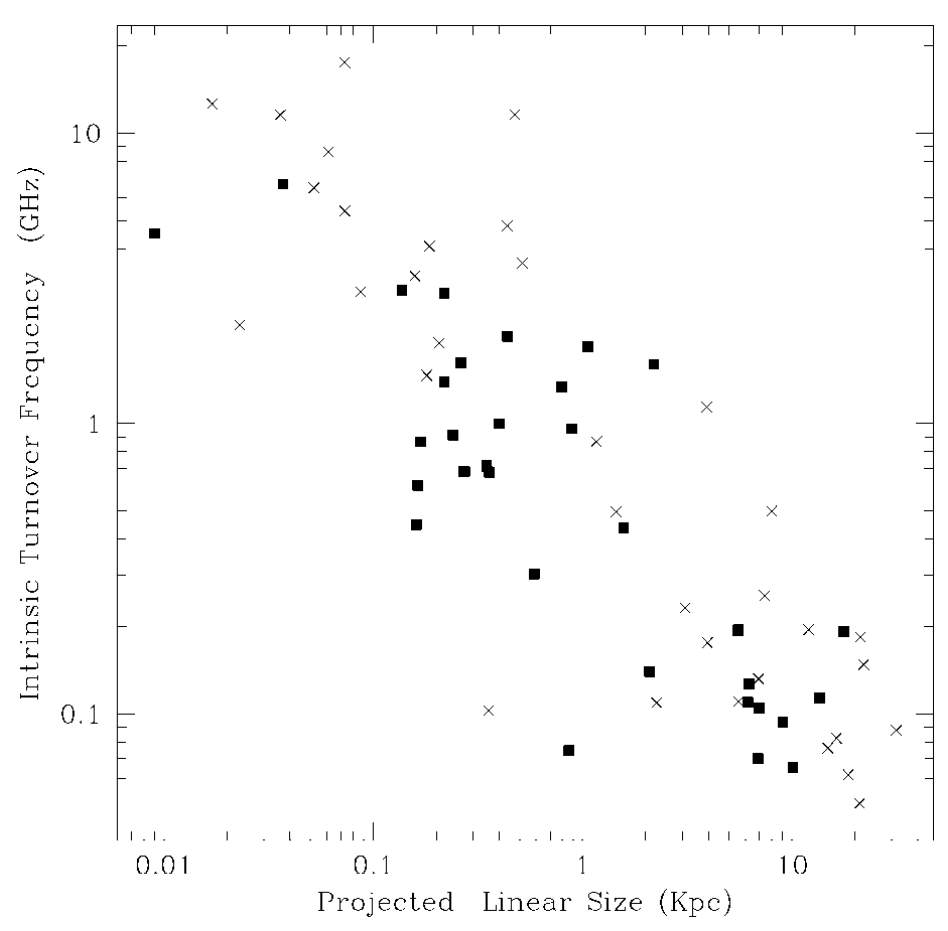

Figure 2.5: Turn over frequency vs the projected linear size of two samples of GPS (Stanghellini et al., 2001) and CSS (Fanti et al., 1995). Quasars are marked by crosses while galaxies are represented by squares. (O'Dea \& Baum, 1997)

According to the previous relationship, Fig.2.5 shows the distribution of the sources in the $\nu_{\text {t.o. }}-$ LS plane. As the source expands its turnover frequency decreases and each source moves downward and right. This is the result of a decreased energy density within the radio emitting region.

However, Bicknell et al. (1997) could reproduce the relationship shown in Fig. 2.5 assuming a FFA model (Bicknell et al., 1997a). In this case the relation is $\nu_{\text {t.o. }} \propto \mathrm{LS}^{-0.96}$. For this reason, the main mechanism that could explain the observed turnover frequencies in compact sources, still remains uncertain and a combination of SSA and FFA may be at work. 


\subsection{Radio Morphology}

Because of their small size CSS, GPS and HFP can be studied with the high resolution interferometry technique which allows an angular resolution of a sub-arcsecond scale. Given their small linear and angular size, the morphological classification of these sources strictly depends on the resolution, the frequency and the sensitivity of the observations, making the correct morphological classification of individual sources rather difficult.

Therefore considering their morphological features, compact radio sources can be divided into four main groups (Fanti et al., 1990):

- DOUBLE: sources which show two well separated components. Sometimes in deep observations a weak flat-spectrum unresolved component is found in between. This is probably the core, usually observable at high frequencies ( $\geq 5 \mathrm{GHz}$ ).

- TRIPLES: three well separated components are visible in these sources. They are not necessary aligned. Deep observations revealed that the central component may host the core.

- JET LIKE: they are elongated structure, usually with a visible flat spectrum core located at one end of the source.

- COMPLEX: this group includes sources whose morphology cannot be classified in any of the previous classes.

The comparison between CSS galaxies and quasars indicate that they have different morphology. CSS quasars show a brighter core and are usually dominated by their bright jets. Most of the CSS/GPS quasars can be included in the Jet-like or Complex group.

On the contrary CSS galaxies are more often Double or Triple sources, with a weak core and, sometimes weak jets. They are usually lobe dominated or hot spot dominated. Fanti et al., (1990) examined the possibility that CSS morphology could be the result of projection effects of large sources. This was excluded by their statistical studied. They concluded that the majority of CSS are intrinsically small radio sources, and that only a small fraction of their sample (roughly $\leq 10 \%$ ) may be large-size source shortened by projection effects. This effect is observed when the relativistic jet is aligned to the line of sight, making the source brighter as a consequence of Doppler boosting and foreshortened due to projection effects.

Another important aspect of compact radio sources is that they often show distorted morphologies. This could be explained by considering the interaction with local dense cloud (Wilkinson et al., 1984). In agreement with this hypothesis, X-ray and IR observations confirmed the existence of different ISM phases inside the host galaxy (Siemiginowska et al., 2005). Also polarization and faraday rotation measures confirm the presence 
of such a dense gas (Cotton et al., 2003) which however is not dense enough to confine the source growth. Additionally, in the standard source model, the hot spot more distant to the core, should be the brighter one. This is due simply to path delay and beaming effects. If we assume that the jet plasma is symmetrically ejected from the central region, we should observe the two hot spots symmetrically placed with respect to the core. This is true only for those radio sources that lay on the plane of the sky and only if the ambient medium has a homogeneous distribution. If these conditions do not hold, the two hot spots would result located at different distance from the central region, resulting in an asymmetric structure of the radio source.

Moreover, even if a geometrical effect could produce quite small observable asymmetries (Orienti et al. 2007) due mainly to the large orientation angle between the radio axis and the l.o.s., in the most cases, compact galaxies, as CSS and GPS result asymmetric because of the ambient medium.

An example of asymmetric GPS is the source J1944+5443. In this case the brighter hot spot is the closer to the core which is contrary to the geometrical model explained above and asymmetry is likely due to the dense, inhomogeneous ambient medium (Polatidis et al., 1999).

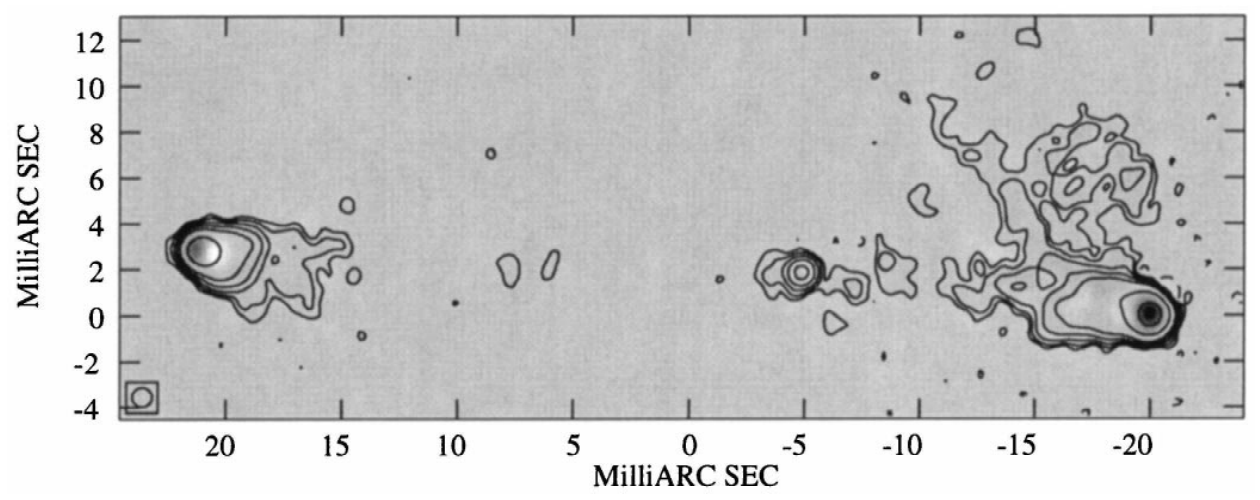

Figure 2.6: The asymmetric GPS J1944+5443 (Polatidis et al., 1999).

\subsection{Polarization}

The synchrotron radiation is known to be linearly polarized with the orientation of the electric vector perpendicular to the orientation of the local magnetic field $\mathrm{H}$. The polarization is strictly related to the wavelength, in particular they are inversely proportional each other. The decreasing polarization (Depolarization) is probably due to the Faraday screen that depolarizes and rotates the polarization vector. 
The Rotation Measure (RM) depends on the ambient medium density and it can be defined as follows:

$$
\mathrm{RM}=0.81 \int \mathrm{n}_{\mathrm{e}} \mathrm{H}_{\|} \mathrm{dl} \quad \mathrm{rad} \mathrm{m}^{-2}
$$

where $\mathrm{n}_{\mathrm{e}}$ is the electron density expressed in $\left(\mathrm{cm}^{-3}\right), \mathrm{H}_{\|}$is the magnetic field (expressed in $(\mu \mathrm{G})$ along the line of sight and $\mathrm{dl}$ is the depth of the Faraday screen. From Eq. 2.19 is possible to obtain the electron density of thermal plasma which produces the observed $\mathrm{RM}$, if $\mathrm{H}_{\|}$is known.

CSS and GPS sources are known to have little or no polarized emission. Due to their small size, the relativistic plasma is completely embedded within the ISM of the parent galaxy. Such ISM may be responsible for the lack of polarization. Cotton et al. (2003) found that small radio sources are unpolarized in the NVSS at $1.4 \mathrm{GHz}$ (Fig. 2.7), while at higher frequencies sources with size of about a few kpc start to show some fractional polarization at progressively smaller projected linear sizes (Fanti et al., 2004).

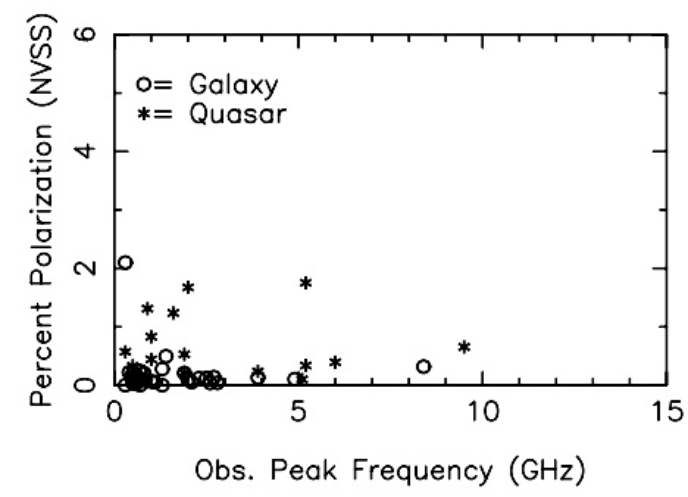

Figure 2.7: Fractional polarization at $1.4 \mathrm{GHz}$ plotted versus the frequency of the peak in the spectrum for a GPS sample (Cotton et al., 2003).

This is consistent with the picture of a small radio source with normal polarization properties whose radio emission is subject to severe Faraday rotation with inhomogeneities on very small scales, producing depolarization.

In addition, an alternative explanation considers either beam depolarization on small scales as a result of a complex magnetic field topology, or, finally, that the source radiation is unpolarized as a result of a tangled magnetic field on very small scales.

In the few cases where it has been possible to achieve polarization detection of CSS/GPS galaxies and quasars, very high $\left(\geq 1000 \mathrm{rad} \mathrm{m}^{-2}\right)$ intrinsic Rotation Measures have been found. This implies that these compact radio sources are generally surrounded by a very dense ionized medium (Fanti, 2004). 


\subsection{Age}

The various classes of compact sources can be interpreted assuming an evolutionary model, known as Self Similar Evolution Model. According to this model each radio source preserves its overall morphology as the radio source expands. HFP will evolve into GPS, which, in turn, will become CSS whose fate is to eventually grow up to reach the size of the extended radio galaxies, like the FR I and FR II. In this model HFP are the youngest radio sources, where the radio activity has just switched on.

In order to demonstrate the validity of the evolutionary model, it is necessary to determine the age of these radio sources, in order to understand if they really are the young phase of old and extended FR I or FR II. To this aim two main approaches are explained in some details.

\subsubsection{Radiative Age}

As previously discussed in Section 2.1, the radiative age can be computed using Eq. 2.15. One disadvantage of this method is that the contributions of the different source components to the overall spectrum, each one with its own spectral shape, are all mixed together. The age determination may be more influenced by the brighter components (as the hot spot). Thus, if we consider a source where the hot spot emission is the dominant one, the source age cannot be directly correlated to the spectral age because inside the hot spot electrons are likely to be re-accelerated.

For this reason considering sources with symmetric shape (emission) we should estimate the spectral age of the source in the following way. We should take into account only those components where probably electrons were not re-accelerated during the source lifetime and where we find a break on the spectral shape. These regions can be found in the innermost edges of the lobes, where electrons were deposited by the hot spot, as the radio activity of the source has just switched on. In addition, in compact objects the magnetic field is more intense if compared to that of the largest radio sources. Indeed the radiative age, according to Eq. 2.15, in compact objects is shorter.

From earlier works about spectral ageing of CSO (Murgia 2003) it has been found that the break frequency decreases from the the hot spot to the source center in the core direction. Particles found in the inner part of the lobes are older because they have been accelerated in the hot spots at an early time. The hunt for the determination of the radiative age is the search for the earliest/oldest relativist particles.

In conclusion, the lobes are the best place to investigate the spectral age. 


\subsubsection{Kinematic Age}

Another method to compute the age of radio sources is related to the measure of the separation speed between the hot spots, thus considering their proper motion: this is called the Kinematic Age method. The kinematic age $t_{\text {kin }}$ can be easily estimated as:

$$
\mathrm{t}_{\mathrm{kin}}=\left(\frac{\mathrm{LS}}{\mathrm{v}_{\mathrm{sep}}}\right)
$$

assuming LS as the total linear size of the source and $\mathrm{v}_{\text {sep }}$ the advance speed of the two hot spots.

As a first approach we could estimate the source age by dividing its overall projected size by the corresponding measured hot spot separation rate known as expansion speed. However, this value can be measured by comparing observations separated by a few (tens) of years. We assume that the source growth happens at a roughly constant speed, but we should keep in mind that it may not represent the true growing rate of the source. For example, there is a phenomenon called 'dentist's drill' (Scheuer, 1982) according to which the hot spots follow a "corkscrew" like path as they move among the ISM and the resulting advance speed is much smaller than the instantaneous value. These may produce an unreliable estimate of the age of the source (Polatidis, 2003). Moreover inhomogeneities of the external medium and hydrodynamical mechanisms could induce other several changes in the source expansion. For example if the external gas is inhomogeneous on the two sides explored by the jets, it may slow down the advance of one hot spot resulting in an asymmetric shape of the source (as described in Section 2.3).

In order to obtain the age of the source, an important tool is the comparison between the spectral and the kinematic age. An example is reported in Murgia (2003). By measuring the spectral break of the CSO J1943+546, Murgia (2003) obtained a spectral age of about $\mathrm{t}_{\mathrm{rad}} \sim 1500 \mathrm{yr}$ which is in good agreement with the kinematic age $\mathrm{t}_{\text {kin }}=1400 \mathrm{yr}$ found by Polatidis \& Conway (2003). 


\subsection{The Models}

Two different models have been proposed to explain the nature of CSS, GPS and HFP. These models should describe all radiative and morphological features of these class of small radio sources, such as their radio luminosity, small size, and the fact that they represent a large fraction of the radio source population in flux density limited samples.

The models explaining the nature of compact radio sources are the Frustration Scenario and the Youth Scenario. They are discussed in the following paragraphs.

\subsubsection{Frustration Scenario}

This model is based on the idea that intrinsically small radio sources will never become large since they will be confined to sub-galactic size by an anomalously dense ambient medium which prevents any growth (van Breugel et al., 1984).

For this reason it is useful to set constrains on the characteristics of the ambient medium, as shown for examples by De Young (1993). Through simple numerical hydrodynamical simulations of a jet propagating in a smooth medium with a constant density, De Young (1993) found that the total gas mass required to confine the radio source is:

$$
\mathrm{M}=10^{9} \mathrm{M}_{\odot}\left(\frac{\mathrm{n}_{0}}{10 \mathrm{~cm}^{-3}}\right)\left(\frac{\mathrm{R}}{1 \mathrm{kpc}}\right)^{3}
$$

where $\mathrm{R}$ is the radius of the medium with density $\mathrm{n}_{0}$.

For $\mathrm{n}_{0} \approx 1 \mathrm{~cm}^{-3}$ the necessary gas mass is $\approx 10^{8} \mathrm{M}_{\odot}$ inside $1 \mathrm{kpc}$. Then De Young (1993) computed that typical CSS/GPS sources, with low-medium luminosity, can be confined only in the case in which the average ISM density is between $1 \leq \mathrm{n} \leq 10 \mathrm{~cm}^{-3}$ (De Young, 1993). The mass of the gas required to confine the sources is about $\approx 10^{12} \mathrm{M}_{\odot}$ which should extends out to $10 \mathrm{kpc}$.

Subsequently, Carvalho et al., (Carvalho, 1994),(Carvalho, 1998) presented a simple analytical model of jet interaction with the ambient medium. Carvalho (1998) obtained that interactions with dense clouds could slow down the jet more than a smooth medium with the same average density. GPS sources could be confined by a clumpy medium with a mass of $\approx 10^{9}-10^{10} \mathrm{M}_{\odot}$, on the scales of hundred pc. However such a dense gas should be observed.

Fanti et al., (2000) analyzed IR observations from the ISO satellite on a representative sample of GPS and CSS sources comparing their IR properties with those of a sample of extended radio sources with similar range of redshift and luminosity. No evidence of different FIR luminosity between the CSS/GPS and extended objects was found, indicating that the gas possibly frustrating the growth of small radio sources is not in the form of cold molecules and dust (Fanti et al., 2000).

Relying on ram pressure studies, the gas mass required to support this hypothesis is about $2 \cdot 10^{10} \mathrm{M} \odot$ within a volume of $\approx 10 \mathrm{kpc}$. It corresponds to a dust mass of $2 \times 10^{8} \mathrm{M} \odot$ which should produce an IR excess of GPS and CSS emission which has never been observed. In addition some studies in the X-ray band (Siemiginowska et al. 
2005) did not show any evidence of an anomalously dense hot $\left(\mathrm{T} \geq 10^{7} \mathrm{~K}\right)$ medium which could produce frustration.

In conclusion, all the observations agree with the fact that:

- There is no evidence of the presence of an anomalously dense ambient medium.

- The gas surrounding the radio sources can only create small local asymmetries and distortion of radio sources shape (see Section 2.5).

- The ambient medium is not able to prevent the sources to grow and expand.

- Compact radio sources are not frustrated large size sources (LSO).

Considering all the previous observational evidence, the frustration model has been ruled out.

\subsubsection{Youth Scenario}

The idea that CSS/GPS/HFP are young radio sources is based on their peaked spectra, compact sizes and the symmetric structures similar to those found in large classical doubles (Phillips \& Mutel, 1982). However, the simplest youth scenario is unable to reproduce the high fraction (15-30\%) of CSS/GPS found in flux density limited catalogues. To solve this problem, evolutionary models which take into account changes in both the growth velocity and luminosity are required (Fanti et al., 1995). So far strong support to the youth scenario comes from both the determination of hot-spot separation velocity in a dozen of the most compact CSO (Polatidis, 2003), and the derivation of the radiative age from the analysis of the source spectra (Murgia 2003), showing that sources with sizes of about $100-500 \mathrm{pc}$ are typically $10^{3}$ years old.

According to the youth scenario, CSO are intrinsically small because they are in an early evolutionary stage. They are expanding within the interstellar medium of the host galaxy, experiencing decreasing external densities as the radio source grows up. 


\section{A realistic model for the Youth Scenario}

In this Section I describe the model proposed by Fanti et al., (1995) based on a the properties of the selected sample of CSS/GPS presented (Fanti et al., 1995). First we assume that the source luminosity is constant. Then, by using a self similar approach, the observed distribution of Linear Size (LS) of CSO can be described with a simple power law:

$$
\frac{\mathrm{dN}(\mathrm{LS})}{\mathrm{d}(\mathrm{LS})} \propto \mathrm{LS}^{-\mathrm{m}}
$$

where the parameter $\mathrm{m}$ depends on the source sample selected (i.e. $\mathrm{m}=0.68$ if lobe dominated MSO are included, or $\mathrm{m}=0.55$ if the sample includes more steep spectrum sources). As the time passes the linear size LS increases:

$$
\frac{\mathrm{dN}(\mathrm{LS})}{\mathrm{d}(\mathrm{LS})} \propto \frac{\mathrm{dt}}{\mathrm{d}(\mathrm{LS})}
$$

and then

$$
\mathrm{v}(\mathrm{LS}) \propto\left[\frac{\mathrm{dN}(\mathrm{LS})}{\mathrm{d}(\mathrm{LS})}\right]^{-1} \propto(\mathrm{LS})^{\mathrm{m}}
$$

this implies that the growth velocity increases with the linear size of the source and therefore with time.

Since a typical compact radio source lies completely inside the host galaxy, the ram pressure of the ISM gas plays a key role, slowing down the source growth.

By simply assuming the equilibrium between the internal pressure $p_{i}$ of the lobes and the ram pressure pram of the ISM we can determine the lobe advance speed Eq. 2.25

$$
\mathrm{p}_{\mathrm{ram}} \simeq \mathrm{n}_{\mathrm{e}} \mathrm{m}_{\mathrm{p}} \mathrm{v}_{1}^{2}
$$

where $\mathrm{n}_{\mathrm{e}}$ is the density of the ISM, $\mathrm{m}_{\mathrm{p}}$ the proton mass, assuming that the gas is mainly composed by hydrogenum, and $\mathrm{v}_{\mathrm{l}}$ is the lobe advance speed. The thermal gas pressure $\mathrm{p}_{\mathrm{i}}$ has to be included, where $\mathrm{p}_{\mathrm{i}}=\mathrm{nkT}, \mathrm{T}$ is the temperature and $\mathrm{k}$ is the Boltzman constant.

At this point, using Eq. 2.25 we obtain the source size as a function of time, if the external density as a function of distance from the centre is known.

We assume a King density profile as shown by X-rays observations (Trinchieri et al., 1986):

$$
\mathrm{n}(\mathrm{r})=\mathrm{n}_{0}\left[1+\left(\frac{\mathrm{r}}{\mathrm{r}_{\mathrm{c}}}\right)^{2}\right]^{-\beta / 2}
$$

where $r_{c}$ is the core radius, defined as the point at which the density is half the central density $\rho_{0}$. 
We obtain that:

$$
\mathrm{n}_{\mathrm{ext}} \approx \mathrm{n}_{0} \quad \text { for } \mathrm{r} \geq \mathrm{r}_{\mathrm{c}}
$$

and

$$
\mathrm{n}_{\text {ext }} \propto(\mathrm{LS})^{-\beta} \text { for } \mathrm{r} \leq \mathrm{r}_{\mathrm{c}}
$$

where $1.5 \leq \beta \leq 2$.

The size of the source can be easily computed at a given time as a function of the central density $n_{0}$, the core radius $r_{c}$ and the exponent $\beta$.

The lobe advance speed is then:

$$
\mathrm{v}_{\mathrm{l}} \propto(\mathrm{LS})^{-1} \text { for } \mathrm{r}>\mathrm{r}_{\mathrm{c}}
$$

and

$$
\mathrm{v}_{\mathrm{l}} \propto(\mathrm{LS})^{[(\beta / 2)-1]} \text { for } \mathrm{r}<\mathrm{r}_{\mathrm{c}}
$$

We have considered a ram pressure model, where

$$
\mathrm{n}_{\mathrm{ext}} \propto(\mathrm{LS})^{-\beta} \text { and } \mathrm{p} \propto(\mathrm{LS})^{-2}
$$

so, the velocity as a function of size is:

$$
\mathrm{v}(\mathrm{LS}) \propto(\mathrm{LS})^{[(\beta / 2)-1]}
$$

For simplicity we have previously assumed the radio luminosity of the source is constant all over its life. For a more realistic study, the luminosity may change with time and with the source size. During the radio source evolution the radio luminosity $(\mathrm{P})$ is described by a power-law of the type:

$$
\mathrm{P} \propto(\mathrm{LS})^{-\mathrm{h}}
$$

If the Luminosity Function is

$$
\frac{\mathrm{dN}(\mathrm{P})}{\mathrm{dP}} \propto \mathrm{P}^{-\mathrm{b}}
$$

we obtain that the size distribution at a given observed $\mathrm{P}$ is:

$$
\frac{\mathrm{dN}(\mathrm{LS}, \mathrm{P})}{\mathrm{d}(\mathrm{LS})} \propto(\mathrm{LS})^{-\mathrm{m}} \cdot \mathrm{LS}^{\mathrm{hb}}=(\mathrm{LS})^{(\mathrm{hb}-\mathrm{m})}
$$

and therefore

$$
\mathrm{v}(\mathrm{LS}) \propto(\mathrm{LS})^{(\mathrm{hb}-\mathrm{m})}
$$


Then, assuming that the external density changes as $\mathrm{LS}^{-\beta}$ for $\mathrm{r}>\mathrm{r}_{\mathrm{c}}$, it is possible to find a relation between the parameters $\mathrm{m}, \mathrm{h}, \mathrm{b}$, and $\beta$ :

$$
-(\mathrm{hb}-\mathrm{m})=(\beta / 2)-1
$$

From the best fit to the bright CSS sample, Fanti et al. (1995) obtained $b=1.5$ and $\mathrm{m} \approx 0.65$. From spectral studies of large-size radio sources, it seems that the velocity $\mathrm{v}$ is roughly independent from LS, which means that:

$$
(\mathrm{hb}-\mathrm{m}) \approx(\beta / 2)-1 \approx 0
$$

which gives

$$
\mathrm{h} \approx 1.1-\beta / 3 \approx 0.5
$$

With the above parametrization, intrinsically small radio sources are generally younger than a few million yr, and are expanding within the ISM of the host galaxy. In this framework they really represent the early stage of classical extended radio galaxies as FR I or FR II.

As presented by Snellen et al., (2000) it is possible to set limits on the luminosity evolution of radio sources by exploiting the radio power - linear size (P-D) diagram as shown in Fig.2.8 (Snellen et al., 2000a).

Sources with large sizes (LS $\leq 1 \mathrm{Mpc})$ and high radio luminosities $\left(\mathrm{P}>10^{26} \mathrm{~W} / \mathrm{Hz}\right.$ at 178 $\mathrm{MHz})$ are rare, suggesting that the luminosity of these sources should decrease quickly as the linear size approaches $1 \mathrm{Mpc}$.

As suggested by Snellen et al., (2000) the presence of a large fraction (10-15\%) of CSS, GPS in radio surveys implies that young radio source luminosity should decrease during their lifetime. In fact, evolved radio sources as FR II should have a luminosity ten times lower with respect to that of CSS/GPS. This aspect can be explained by a decreasing radiation efficiency as the source size increases. As shown in Fig. 2.8 the radio luminosity decreases as the CSS-phase starts.

In conclusion,

- GPS and CSS share the same morphologies of large doubles sources as FR II with the only difference that CSS/GPS have smaller size.

- The host galaxies of CSO are similar to those of FRI and FRII. There are no proofs of the presence of enough dense gas in these kind of galaxies that could confine CSO. (see Section 2.6.1)

- Multi-epoch observations of the hot spot advance speed represent one of the most important proof of the source expansion through the ISM. 


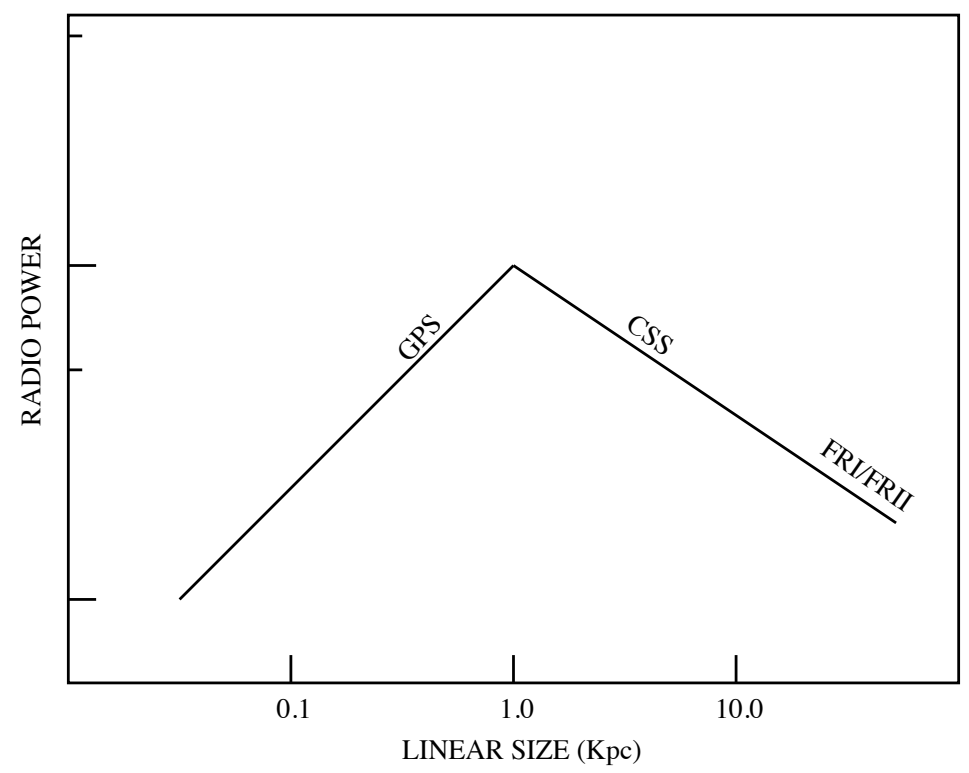

Figure 2.8: Proposed evolutionary scenario (Snellen et al., 2000a).

- The estimation of the radiative age of CSO confirms that these compact source are very young object.

In conclusion, both the estimated radiative and kinematic age well agree with the Youth Scenario, confirming that compact sources are truly young objects, and that they are probably the progenitors of the extended radio galaxies as FR II. In agreement with the youth scenario and considering all the features of CSO the common accepted idea is the following.

HFP are considered as "newborn" radio sources, where the radio activity has just switched on. Then it is thought they would grow and evolve as GPS, thus with a turnover at lower frequency and higher luminosity and with a larger structure. Then GPS would grow and expand as CSS, decreasing their luminosity and their radio power. Finally, CSS would become LSO, with a low frequency turn over and a morphological extended structure, which would exceed the host galaxy boundaries of many orders of magnitude. 
A simple representation of this Evolutionary Sequence is shown in Fig. 2.9. In this plot Orienti et al., (2013) collected the data from various samples of CSS, GPS and HFP, considering the linear size (LS) and the total luminosity $\left(\mathrm{L}_{\text {tot }}\right)$. It is clera that this distribution well reflects the evolutionary model proposed by Snellen et al. (2000).

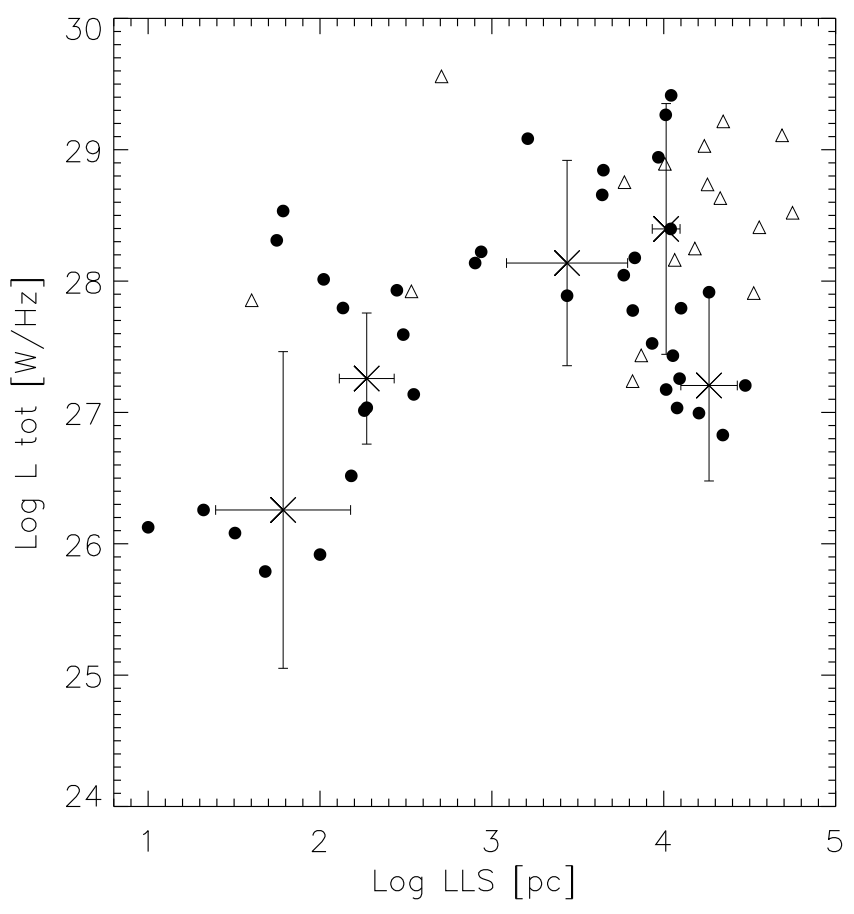

Figure 2.9: Total luminosity at $375 \mathrm{MHz}$ vs. LS of CSs/GPS/HFP samples. Circles represent galaxies and triangles are quasars (Orienti \& Dallacasa, 2013). 


\section{Chapter 3}

\section{Radio Observations}

The aim of this Chapter is to describe the radio data sets used in this work and the calibration procedure. The instrument used to take the observation, is described in Section 3.1. The data set is shown in Section 3.2. Then, the procedure of calibration is describe in Sections 3.3 and 3.4.

\subsection{Very Long Baseline Interferometry}

In the 1950s Interferometry techniques was introduced in order to improve the resolution of radio observation in order to resolve the source structure.

The Very Long Baseline Interferometry (VLBI) is a technique according to which the antennas of the interferometry are not physically connected and in particular they are located across the Earth. So the signals received by each antenna are recorded and then correlated. The measurements are made independently at the individual antennas of the interferometer. The data are recorded on storage media together with precise time marks using atomic clocks.

The motivation that has brought the development of this technique is the angular resolution that can be achieved. The resolution, $\theta$, of an interferometer is:

$$
\theta \approx \frac{\lambda}{\mathrm{d}}
$$

where $\mathrm{d}$ is the maximum separation between the antennas, called also baseline, and $\lambda$ is the wavelenght. Since the VLBI antenna are so sparsely located, thus the maximum baseline that can be reached is the order of $10^{4} \mathrm{~km}$. This produce an angular resolution of the order of (sub)-milliarcsecond scale (depending on the frequency) that allows us to detect very small and compact object (parsec scale). 


\subsubsection{Very Long Baseline Array}

The Very Long Baseline Array (VLBA) is an interferometer of the National Radio Astronomy Observatory (NRAO) consisting of 10 identical parabolic antennas with a diameter of $25 \mathrm{~m}$ (Table 3.1). The VLBA observes at wavelengths from $90 \mathrm{~cm}$ to $3 \mathrm{~mm}(327 \mathrm{MHz}$ to $96 \mathrm{GHz}$ ) in eight discrete bands (P, L, S, C, X, U, K, Q and W). The antennas are located across the United States. The maximum baseline is $\approx 8600 \mathrm{~km}$ between Mauna Kea (the westernmost antenna) and Saint Croix (the easternmost antenna), while the shortest baseline is $\approx 200 \mathrm{~km}$ between Pie Town and Los Alamos in New Mexico. The signals from each antenna are recorded and the information is time-stamped using atomic clocks.

The resolution of the VLBA is:

$$
\theta_{\mathrm{HPBW}} \approx 2063 \times \frac{\lambda}{\mathrm{B}_{\max }} \operatorname{mas}
$$

where the wavelength $\lambda$ is expressed in $\mathrm{cm}$ and the maximum Baseline $\mathrm{B}_{\max }$ in $\mathrm{km}$ (Wrobel, 1995). A description of the position of each antenna is given in Fig.3.1. The location of the VLBA antennas provide a good coverage of both short and long baselines, allowing a proper observation of both extended and compact objects. Sometimes, it is convenient to add to the VLBA one VLA antenna in order to increase the number of short baselines (see Section 3.5). Table 3.2 gives the resolution of the VLBA for each bandwidth. 


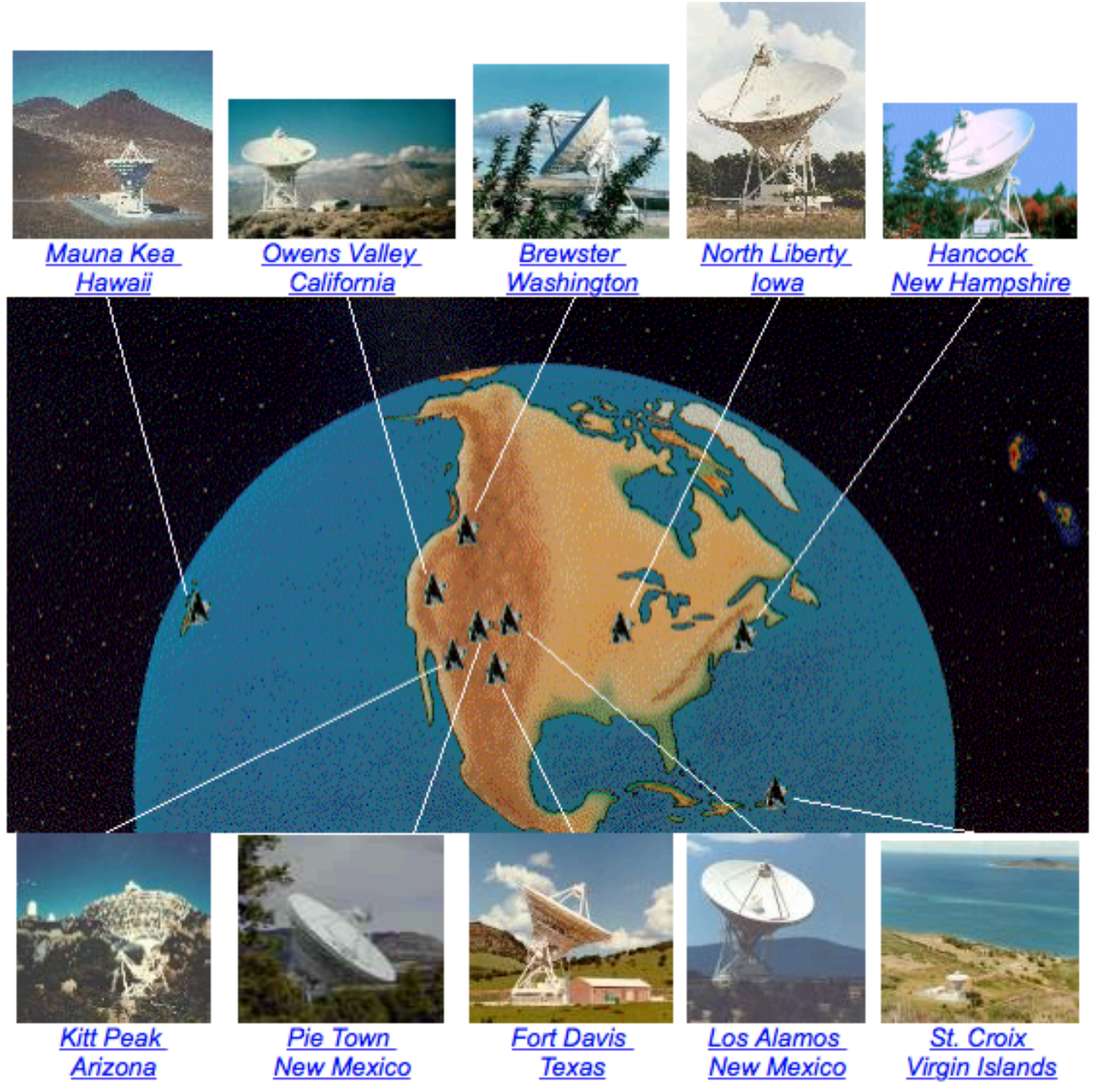

Figure 3.1: The location of the VLBA sites. 


\begin{tabular}{|cccccc|}
\hline Name & U.S.A. state & \multicolumn{2}{c|}{ Geographic coordinate } & Elevation (M) & Code \\
\hline St. Croix & U.S. Virgin Islands & $17.75652^{\circ} \mathrm{N}$ & $64.583760^{\circ} \mathrm{N}$ & -15 & $\mathrm{SC}$ \\
\hline Hancock & New Hampshire & $42.93362^{\circ} \mathrm{N}$ & $71.986810^{\circ} \mathrm{N}$ & 296 & $\mathrm{HN}$ \\
\hline North Liberty & Iowa & $41.77165^{\circ} \mathrm{N}$ & $91.574133^{\circ} \mathrm{N}$ & 222 & $\mathrm{NL}$ \\
\hline Fort Davis & Texas & $30.63522^{\circ} \mathrm{N}$ & $103.94483^{\circ} \mathrm{N}$ & 1606 & $\mathrm{FD}$ \\
\hline$\underline{\text { Los Alamos }}$ & New Mexico & $35.78233^{\circ} \mathrm{N}$ & $106.25568^{\circ} \mathrm{N}$ & 1962 & $\mathrm{LS}$ \\
\hline Pie Town & New Mexico & $34.30107^{\circ} \mathrm{N}$ & $108.11912^{\circ} \mathrm{N}$ & 2365 & $\mathrm{PT}$ \\
\hline Kitt Peak & Arizona & $31.95625^{\circ} \mathrm{N}$ & $111.61236^{\circ} \mathrm{N}$ & 1902 & $\mathrm{KP}$ \\
\hline Owens Valley & California & $37.23176^{\circ} \mathrm{N}$ & $118.27714^{\circ} \mathrm{N}$ & 1196 & $\mathrm{OV}$ \\
\hline Brewster & Washington & $48.13117^{\circ} \mathrm{N}$ & $119.68325^{\circ} \mathrm{N}$ & 250 & $\mathrm{BR}$ \\
\hline Mauna Kea & Hawaii & $19.80159^{\circ} \mathrm{N}$ & $155.45581^{\circ} \mathrm{N}$ & 3763 & $\mathrm{MK}$ \\
\hline
\end{tabular}

Table 3.1: The VLBA stations. Column 1: name; Column 2: location; Columns 3 and 4: longitude and latitude; Column 5: the elevation; Column 5: the code-name.

\begin{tabular}{cccc}
\hline Band & $\lambda(\mathbf{c m})$ & $\nu(\mathbf{G H z})$ & $\theta_{\text {HPBW }}(\mathbf{m a s})$ \\
\hline $\mathbf{P}$ & 90 & 0.333 & 22 \\
$\mathbf{P}$ & 50 & 0.599 & 12 \\
$\mathbf{L}$ & 21 & 1.427 & 5 \\
$\mathbf{S}$ & 13 & 2.310 & 3.2 \\
$\mathbf{C}$ & 6 & 4.990 & 1.4 \\
$\mathbf{X}$ & 3.6 & 8.32 & 0.85 \\
$\mathbf{U}$ & 2 & 14.982 & 0.47 \\
$\mathbf{K}$ & 1 & 29.972 & 0.32 \\
$\mathbf{Q}$ & 0.7 & 42.827 & 0.17 \\
$\mathbf{W}$ & 0.3 & 99.9 & 0.12 \\
\hline
\end{tabular}

Table 3.2: VLBA resolution in different bands. 


\subsection{The Dataset}

In this work I analyzed the observations of two CSO, J1944+5448 and J0111+3906 (alias B0108+388), performed on April 27, 2008 using the VLBA. In addition to the VLBA an antenna of the VLA participated in the observations in order to improve the $u v$-coverage and increase the number of short baselines. In this case the shortest baseline is $\approx 70 \mathrm{~km}$. The correlation was performed at the VLBA correlator in Socorro. The two sources were observed for a time of about 26 hours at three frequencies. In particular J1944+5448 was observed at $\mathrm{C}$ and $\mathrm{X}$ band $(4.8$ and $8.4 \mathrm{GHz}$ ), while J0111+3906 was observed at L, C and $\mathrm{X}$ band (1.4, 4.8 and $8.4 \mathrm{GHz}$ ).

Each frequency band is divided into four IF with a bandwidth of $\approx 8000 \mathrm{khz}$ and each IF is split into sixteen channels of $500 \mathrm{kHz}$ reach as shown in Table 3.2.

Fig. 3.2 shows the different $u v$-coverage in each band of our observations. The scales are different for each plot, this is due to the different frequencies band used which means different resolution, as in the case of the $\mathrm{X}$ band for example, whose $u v$-plot is extend to 200 mega-wave length.

The multifrequency observations are necessary in order to investigate both the spectral and morphological features of these sources (see Section 2.4 and 2.5). The high resolution achieve in $\mathrm{C}$ and in $\mathrm{X}$ band, is adeguate for determining the separation between the core and the hot spots, and thus for investigating the kinematic age (see Section 2.7.2). The availability of three band observations provides a very useful tool to analyze the spectral features of these sources.

A description of the "observational" details of the targets are presented in Table 3.4.

\begin{tabular}{ccc}
\hline Band & IF & $\begin{array}{c}\text { Frequency } \\
\nu(\mathbf{G H z})\end{array}$ \\
\hline \multirow{4}{*}{$\mathbf{L}$} & 1 & 1.4114 \\
& 2 & 1.4194 \\
& 3 & 1.6314 \\
& 4 & 1.6394 \\
\hline \multirow{4}{*}{$\mathbf{C}$} & 1 & 4.53899 \\
& 2 & 4.54699 \\
& 3 & 4.87699 \\
& 4 & 4.88899 \\
\hline & 1 & 8.11099 \\
$\mathbf{X}$ & 2 & 8.11899 \\
& 3 & 8.48099 \\
& 4 & 8.48899 \\
\hline
\end{tabular}

Table 3.3: The three bands of our observations L, C and X. 


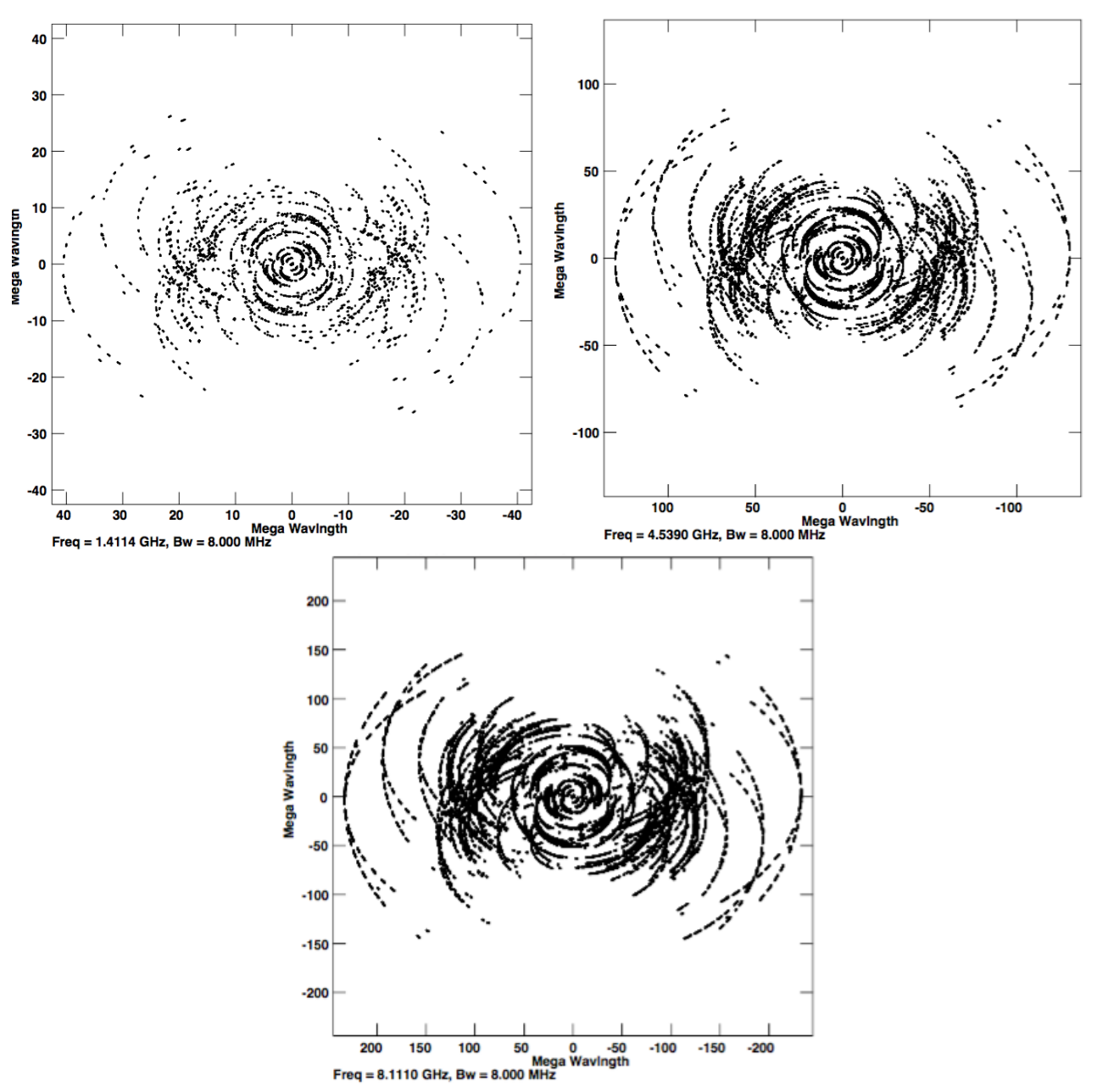

Figure 3.2: The uv-coverage of our observation. top left: L band, top right: $\mathrm{C}$ band, bottom: X band. 


\begin{tabular}{lcc}
\hline & $\mathbf{J 1 9 4 4 + 5 4 4 8}$ & $\mathbf{J 0 1 1 1 + 3 9 0 6}$ \\
\hline Redshift & 0.263 & 0.668470 \\
Luminosity Distance (Mpc) & 1346.4 & 4074.7 \\
Scale (kpc/") & 4.092 & 7.096 \\
RA & $19 \mathrm{~h} 44 \mathrm{~m} 31.5 \mathrm{~s}$ & $01 \mathrm{~h} 11 \mathrm{~m} 37.3 \mathrm{~s}$ \\
DEC & $+54 \mathrm{~d} 48 \mathrm{~m} 07 \mathrm{~s}$ & $+39 \mathrm{~d} 06 \mathrm{~m} 28 \mathrm{~s}$ \\
Type & Galaxy & Galaxy \\
\hline
\end{tabular}

Table 3.4: The observational parameters of the targets.

\subsection{Introduction to Calibration}

An interferometer samples a quantity which is called the Visibility Function. The visibilities are corrupted by the instrumentation and the atmosphere and need to be corrected. The complex visibility, $\mathrm{V}_{\mathrm{obs}}^{\mathrm{ij}}$ is equal to the real visibility $\mathrm{V}_{\text {true }}^{\mathrm{ij}}$ multiplied by a complex gain factor $G$ that describes the Antenna response :

$$
\mathrm{V}_{\text {obs }}^{\mathrm{ij}}=\mathrm{V}_{\text {true }}^{\mathrm{ij}} \mathrm{G}^{\mathrm{i}} \mathrm{G}^{\mathrm{j}}
$$

The two parameters $G^{i}$ and $G^{j}$ are the complex gains of the antenna $i$ and $j$ respectively. They include a series of factors like electronics and signal propagation. The initial flux density scale is fully arbitrary, so we need to set the amplitudes to the proper value.

The complex gain $\mathrm{G}$ can be generally split in to two terms, the amplitude a and the phase $\theta$. Then we can re-write Eq. 3.3 as:

$$
A_{i j}^{\text {obs }} e^{\text {obs }}=A_{i j}^{\text {true }} a_{i} a_{j} e^{i\left(\theta_{i}-\theta_{j}+\theta_{i j}^{\text {true }}\right)}
$$

where $\mathrm{A}_{\mathrm{ij}}^{\text {true }}$ and $\mathrm{A}_{\mathrm{ij}}^{\text {obs }}$ are the true and measured visibility on the $\mathrm{i}-\mathrm{j}$ baseline, both are function of time and frequency. The calibration is the process that allows us to determine $\mathrm{G}$ as a function of time (both its phase and amplitude terms) in order to obtain the true visibility. 


\subsection{VLBA data calibration}

The data calibration and analysis of the two CSO (Section 3.2), were performed using the Astronomical Image Processing Software package (AIPS). This software is composed by TASKS in which we can select parameters depending on the analysis we want to perform.

The data of our observations contain the complex visibilities of each source. Each file (which is a multi source file) holds the information about the source coordinates, the time range, the observing frequencies, the date and the number of visibilities of the target and the calibrators, (Fig. 3.3). Moreover, there are different TABLES associated with the multi-source dataset. Some tables are attached to each file and contains a priori information and others are created during the calibration procedure.

Each step of the calibration that we used for our VLBA observation, is described in the following section.

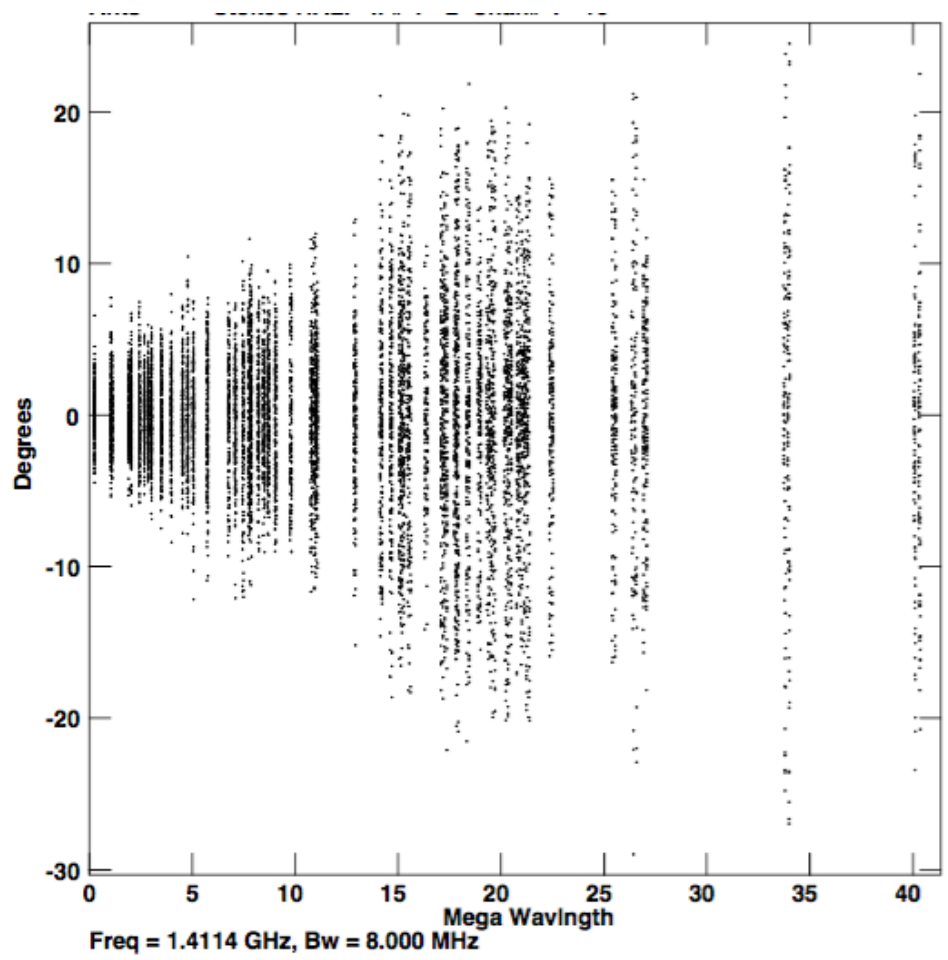

Figure 3.3: The phases of the calibrator DA193 in L band. 


\subsubsection{A priori calibration}

The calibration steps described in this section uses a priori information about the performance of the antennas. After loading the data into AIPS it is useful have a look at the TY table (temperature system table, which is attached to each multi-source file) by using the task SNPLT in order to check anomalously high temperature which may indicate possible bad data. As shown in Fig. 3.4 the bad temperature data need to be flagged. This operation is made with the task SNEDT.
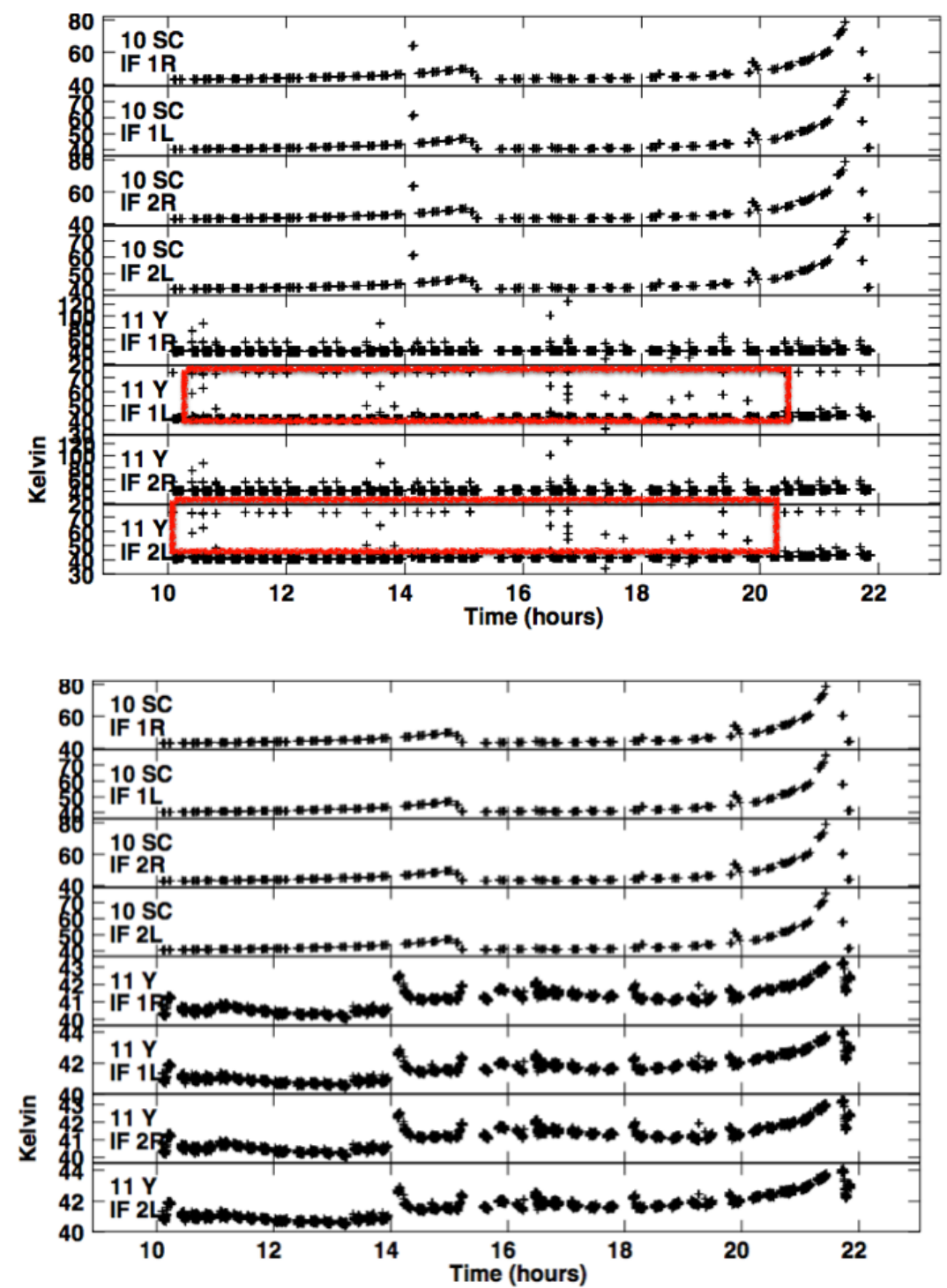

Figure 3.4: An example of the temperature system plot of two antennas. top: before the flagging, there are several bad point (red boxes). bottom: the bad points are removed from the TY table. 
After the flagging of the bad data a new TY table is created with only the good values. Then we must make corrections for the Earth rotation. This is a fundamental step when calibrating VLBA data because in this case the antennas are located at very different latitude each other. The VLBI correlator must use measurements of the Earth Orientation Parameters (EOPs). These parameters change slowly with time and therefore they must be continually updated. This correction is stored in a new CL table.

The a priori calibration of the amplitudes is done by the utility VLBACALA which uses the GC and TY tables. Then it is possible to correct the amplitude for the atmospheric opacity above each antenna in the case of high frequency observations. Then we correct for the parallactic angle using the procedure VLBAPANG. Both the amplitude and phase calibration are kept in the new CL table. The solution corrections that are stored in an SN table can be applied to the data by using the task CLCAL. Additionally, the instrumental phases can be corrected by using the task VLBAMPCL. Also in this case I examined the visibilities to look for bad data, (points that have anomalous amplitude or phase values) which need to be removed (an example is shown in Fig. 3.5). An example of the calibrated phases and amplitudes in $\mathrm{C}$ band is shown in Fig. 3.6.

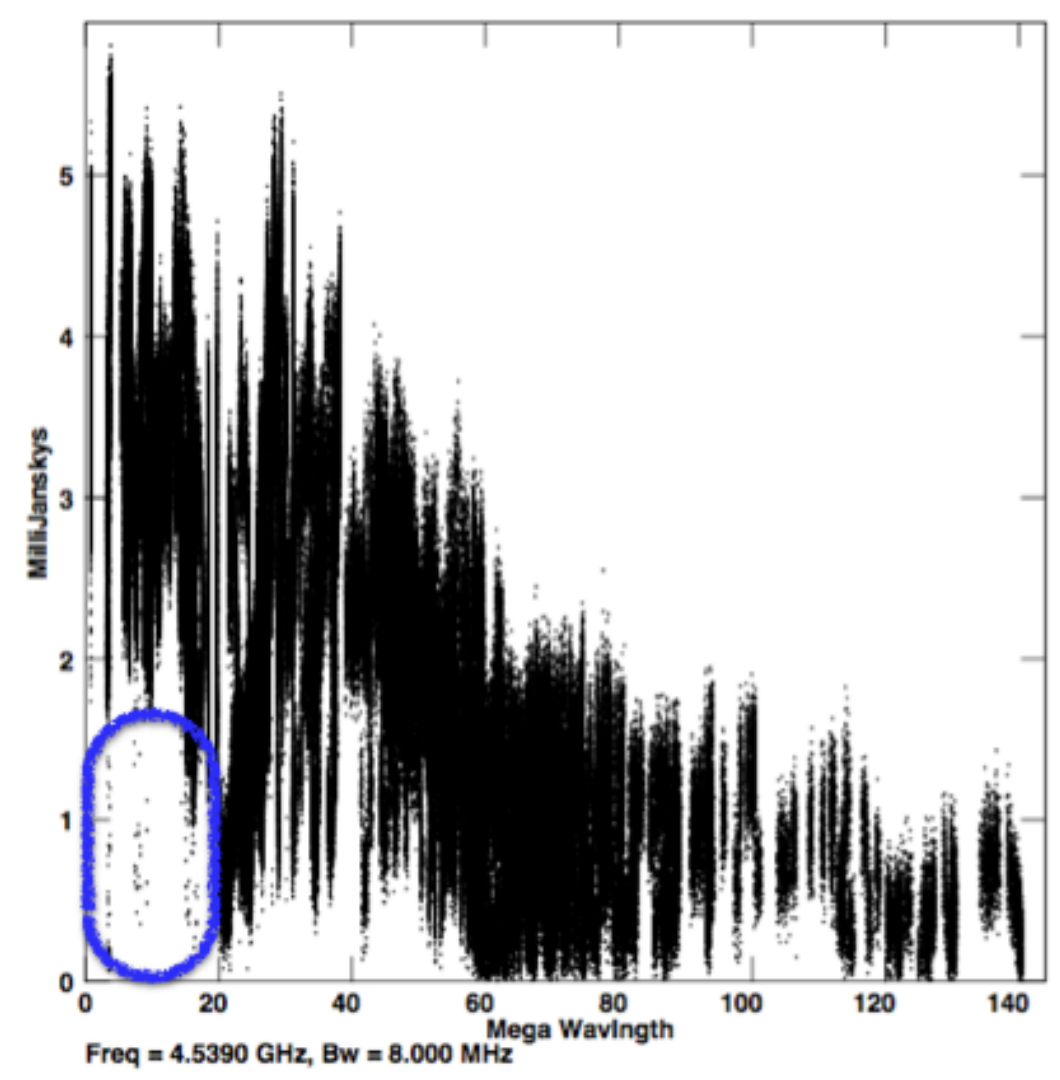

Figure 3.5: An example of the visibilities that need to be flagged. 

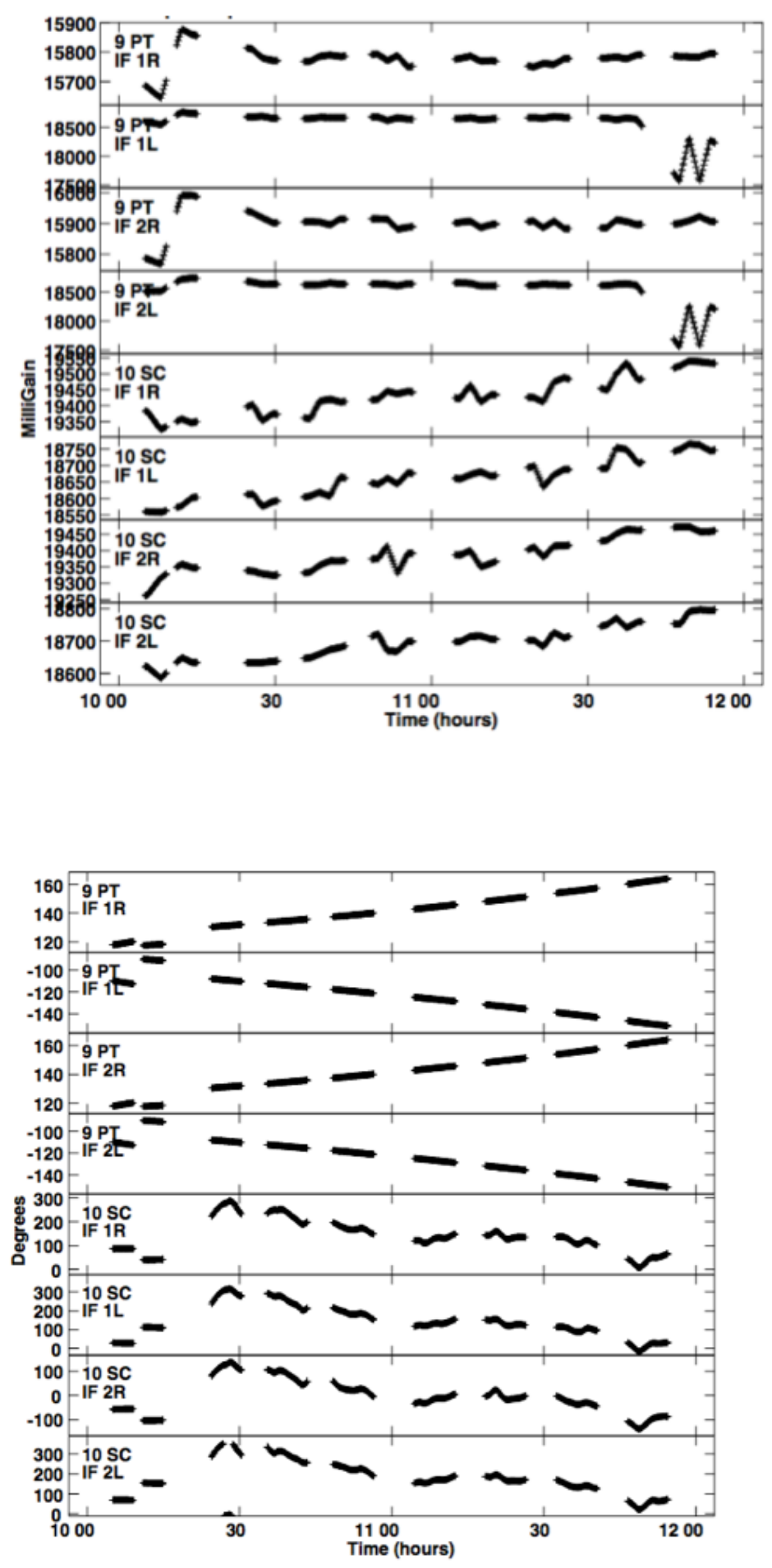

Figure 3.6: An example of the corrections of the amplitudes (top) and of the phases (bottom) after the a priori calibration. The plot is related only to a pair of antennas (Pie Town and Saint Croix) for a selected time range. 


\subsubsection{Fringe fitting}

After correlation the phases the data still exhibits residual fringe rates and delays. Before imaging, these residuals should be removed to permit data averaging in time and, for a continuum observation, in frequency. The process of finding these residuals is referred to as Fringe Fitting (Cotton, 1995a) and it is typical of the VLBI calibration process. In fact in VLBI observations we do not know a priori where the interference fringes are in the fringe-rate/delay space because of the following factors:

- independent clocks,

- changes of baseline geometry due to Earth tides etc.,

- different geometric paths over the telescopes,

- uncertainty in the target position.

Therefore the correlation has to be done for several trial delays which are closely spaced in time. A strong source (fringe finder) is used to find the fringes. If we represent visibilities by vectors, phases by the directions of arrows, the situation is the one shown in Fig. 3.7: Global fringe fitting is simply a generalization of the phase self-calibration technique (see

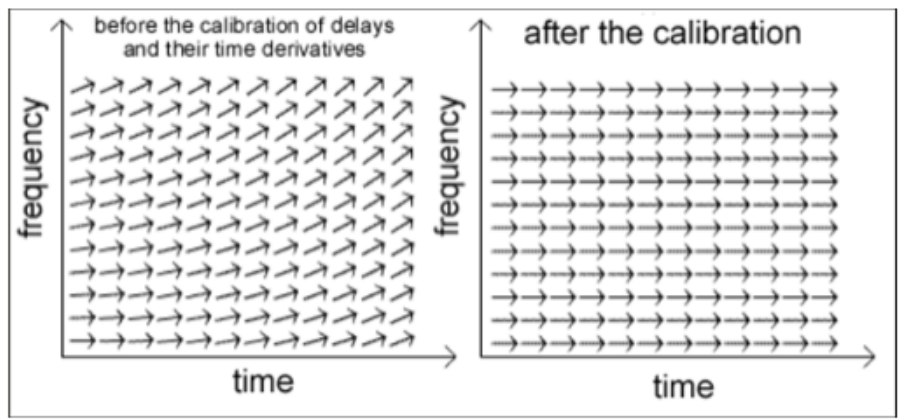

Figure 3.7: A schematic example of the delay correction. The visibilities are represented by vectors and their direction defines the phases (credits: National Astronomical Observatory of Japan).

Section 4.2.5), as during a global fringe fit the difference between model phases (phases=0) and measured phases are minimized by solving for the station-based instrumental phase, its time slope (the fringe rate), and its frequency slope (the delay). Moreover, we should take into account the possibility that the source has a complex morphology. In this case its structure may contribute significantly to the residual delays and rates, and thus it is necessary a second-round of fringe-fitting. In this case, a model of the source (an image) in the second fringe-fitting is used in order to obtain more accurate values of the delay and rate errors. Global fringe fitting in AIPS is done with the task FRING. The fringe-fitting procedure is made separately for calibrators and each target source. 


\subsubsection{Bandpass Calibration}

Bandpass calibration is the process of measuring and correcting the frequency-dependent part of the gains (see section 4.1). With this procedure we determine the variations of both the phases and the amplitudes with the frequency and account for slow timedependency of the bandpass response. The bandpass calibration makes all channels (of each IF) normalized at a single value. If visibilities have not a flat response either in terms of amplitude or phase, the gain is different for every channel of the bandpass of each IF. These variations in the complex gains must be corrected before the visibilities from individual frequency channels are coherently averaged. In particular the Bandpass resolves the spectral profile of the filter which influences both amplitudes and phase of each channel for each IF. Thus we consider the flux density (S) of a point-like/unresolved source imposing that the phases $\phi=0$ and the amplitudes $\mathrm{A}=\mathrm{S}$. We used the first calibrator (DA193) to estimate the correction to apply to the bandpass. The bandpass (BP) table created is then attached to the dataset. In Fig. 3.8 the effect of the bandpass calibration is shown.
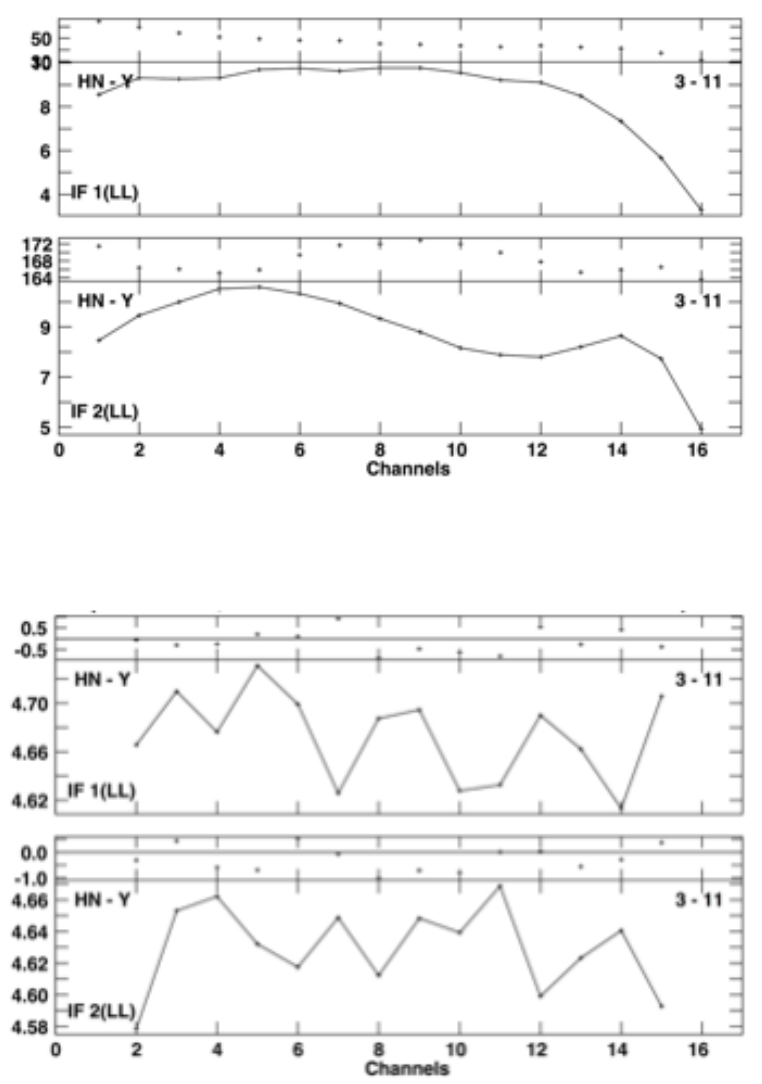

Figure 3.8: Example of the bandpass calibration of the band C for the calibrator DA193. top the phases and the amplitudes before and bottom after the bandpass calibration, respectively. The two plots show the LL (IF1) and LL (IF2) polarization. 
After the bandpass calibration we split the multi-source file using the task SPLIT. SPLIT applies the calibration and writes an UV data file for each source. In addition we decided to merge the channels of each IF in order to improve the signal-to-noise-ratio $(\mathrm{S} / \mathrm{N})$ and speed up the subsequent procedure of imaging. Moreover we averaged the data in time considering the integration time of each observations (4 seconds), using the task UVAVG . Then it is convenient to have a look at the visibilities in order to check for bad data points. This editing is performed with the task IBLED which is an interactive task and allows one to remove bad data points for each IF for each baseline.

\subsubsection{Imaging and self calibration}

After fringe fitting, bandpass, and editing, the phases on a VLBA target source can still vary with time since they have been computed on timescales of the order of a few minutes. Most of these variations are due to inadequate removal of station-based atmospheric phases, but some variations may be due to an inadequate model of the source structure during fringe fitting. If the VLBA target source is sufficiently strong then it is possible to reduce these phase fluctuations by looping through cycles of Fourier transform imaging and deconvolution, combined with phase self-calibration in a time interval shorter than that used for the fringe fit (Cornwell \& Fomalont, 1999). The process of imaging and self-calibration is described as follows.

\section{Cleaning}

As shown in Section 3.4 the radio images suffer the partial uv-coverage which generates lobes of grating which affect the resulting images. For this reason it is necessary to clean the maps and reconstruct the images of the targets. This procedure is called Cleaning Restore and it is done in AIPS using the tasks IMAGR. This method is based on the idea that we can interpret the brightness of an extended source in a finite number of point-like sources.

The cleaning procedure done by the task IMAGR is based on the Clark Clean (1980). This method works only on the visibility plane (Fourier plane) and consists of cyclic steps (Fig. 3.9):

1. For each cycle it use a Högbom Clean (1974) ${ }^{1}$ in order to find peaks on the map and removes the $10 \%$ of the peak flux.

2. It calculates the Fourier Transformed (FT) of each component $\delta$ on the $u v$-plane. Then it subtracts the visibilities and the lobes of grating associated with that component from the observed visibilities.

3. It calculates FT of the subtracted visibilities of step (2) and it obtains a new dirty map.

\footnotetext{
${ }^{1}$ The Hogbom Clean (1974) operates directly on the image visbility, while the Clark Clean makes use
} of the FT, working on the visibility plane. 
4. then it re-starts from the step (1) repeating the cycle until it reaches a fixed number of $\delta$ components.

The point like components $(\delta)$ found by the CLEAN (termed Clean Component, CC) are considered a model of the source. By applying the FT to the $\delta$ components we obtain the visibility model which is compared to the real data. The Clark clean allows us to subtract the components from all the sky and not only from the portion covered by the image plane as the Hogbom Clean. The Clark Clean calculates $\mathrm{V}_{\text {observed }}-\mathrm{V}_{\text {model }}=\mathrm{V}_{\text {residual }}$ and then operates the FT on the residual visibilities obtaining a new residual map where the clean restarts as described in Fig. 3.9 .

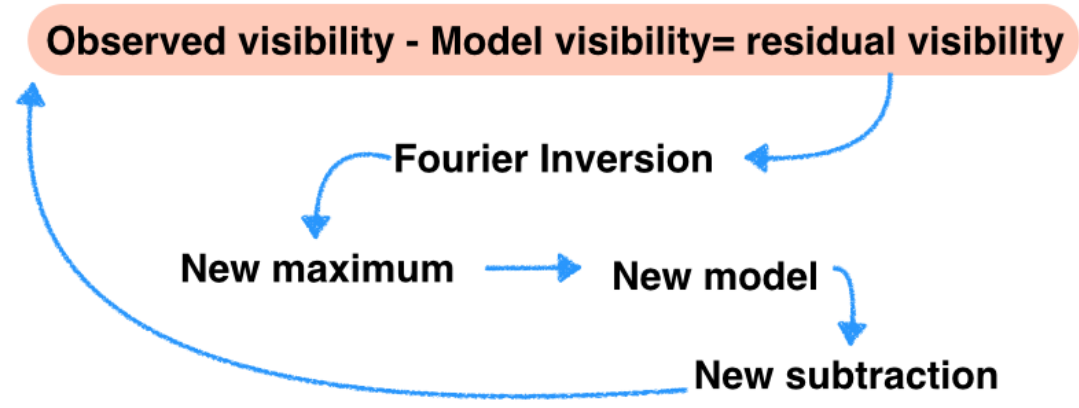

Figure 3.9: A scheme of the Clark clean cycle.

Because of the time necessary to calculate the Fourier Transform, it is possible to constrain the Clark clean to selected boxes of the images. The algorithm looks for the $\delta$ components inside the user selected boxes and removes their contribution from the whole visibility.

We should keep in mind that the images are sampled in pixels whose angular size is chosen to be a fraction $(<1 / 2)$ of the Half Power Beam Width (HPBW) (from the "Nyquist sampling Theorem"). The size of the images depends on the sky surface brightness distribution. In IMAGR we can choose the number of iterations (NITER), the size of the pixel in arc-second (CELLSIZE) and the size of the images itself (IMSIZE).

The final part of this procedure, called RESTORE consists on reconstructing the image adding all the components $(\delta)$ found by the clean to the residual map which have been convolved with a clean beam, that is the same of the dirty map but without the sidelobes (a Gaussian beam). 


\section{Self-Calibration}

If we look back to Eq.3.4 (Section 3.3) for the phases only we obtain:

$$
\theta_{\mathrm{ij}}^{\text {obs }}=\delta \theta_{\mathrm{i}}-\delta \theta_{\mathrm{j}}+\theta_{\mathrm{ij}}^{\text {true }}
$$

which is called "Closure Phases relationship" and describes the variations of the observed phases ( $\delta \theta_{\mathrm{i}}$ and $\delta \theta_{\mathrm{j}}$ are the error on the phases for the $\mathrm{i}$ and $\mathrm{j}$ antenna respectively). The reason for the term "closure" can be seen by summing the phases of three antennas i, $\mathrm{j}$ and $\mathrm{k}$ (a closed triangle) and noting that $\delta \theta_{\mathrm{i}}, \delta \theta_{\mathrm{j}}, \delta \theta_{\mathrm{k}}$ appear twice but with opposite sign and can be cancelled:

$$
\Phi_{\mathrm{c}}=\theta_{\mathrm{ij}}+\theta_{\mathrm{jk}}-\theta_{\mathrm{ki}}=\theta_{\mathrm{ij}}^{\prime}+\theta_{\mathrm{jk}}^{\prime}+\theta_{\mathrm{ki}}^{\prime}=\Phi_{\mathrm{c}}^{\prime}
$$

where $\Phi_{\mathrm{c}}$ is the observed closure phase which coincides with the true one $\hat{\Phi}_{\mathrm{c}}$ when the errors are zero. Therefore the closure phase (Eq. 3.6) may be a good observable but unfortunately there are not enough equations-information to solve for it. In fact considering $\mathrm{N}$ antennas, we have $\mathrm{N}(\mathrm{N}-1) / 2$ baselines and $(\mathrm{N}-1)(\mathrm{N}-2) / 2$ closure relationships the system is unsolvable which implies that information about the phases is incomplete. For this reason it is convenient to adopt a different strategy taking advantage of the data themselves. This method is usually defined as a posteriori calibration and it is based on a self-calibration process.

As previously mentioned, a-priori calibration determines amplitude and phase solutions which are first-order approximations of the antenna performance. Such solutions are sampled on time intervals of a few minutes. Such calibration may be improved and determined on a much shorter timescale by using Self-Calibration. Phase self-calibration just involves minimizing the difference between observed phases and model phases by solving for station-based instrumental phases $\delta \theta_{\mathrm{i}}$ (Walker, 1995). After removing of these instrumental phases, the improved visibilities are used to generate an improved set of model phases, usually based on a new de-convolved and clean image. This process is iterated several times until the phase variations are substantially reduced. Self-calibration should only be done if the VLBA target source is detected with sufficient signal-to-noise in the self-calibration time interval, and if absolute positional information is not needed. The self calibration procedure is made in AIPS by means of task CALIB, with a model provided by the clean components (CC or $\delta$ components).

Moreover at the very end of this iterative process we applied amplitude calibration once, where we consider a larger solution interval than the one used in the phase selfcalibration (that is based on the integration time). As shown in Fig. 3.10 the model is in good agreement with the data after the self calibration procedure.

A scheme of the process adopted in this part of the data reduction is described in Fig. 3.11 . 


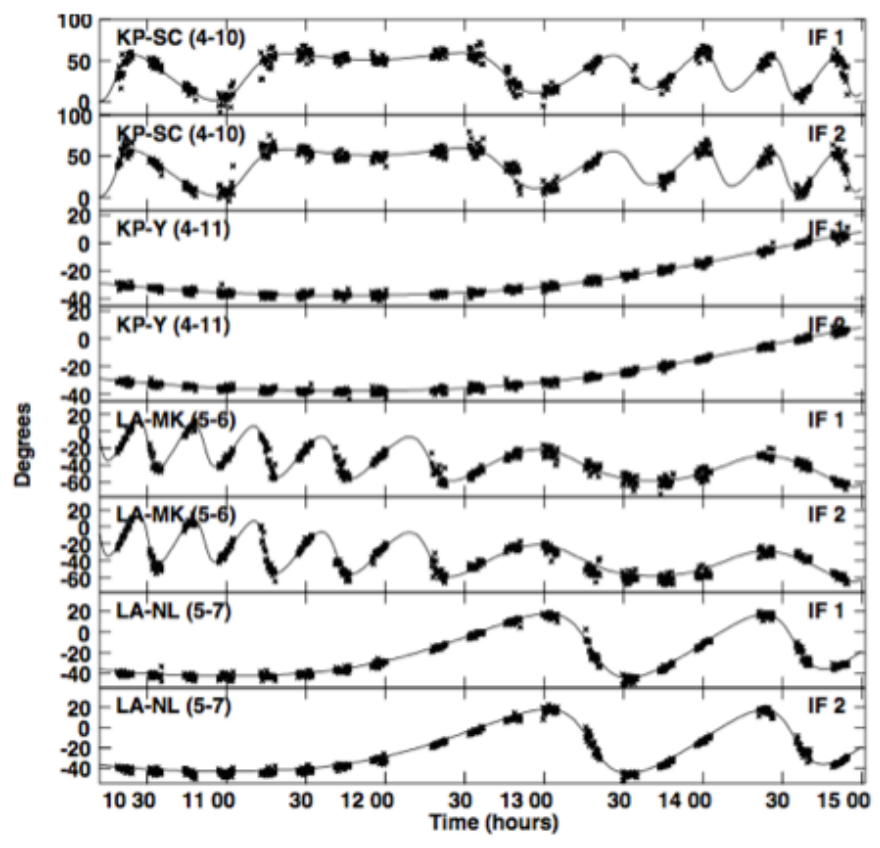

Figure 3.10: An example of the phases of the source J1944+5448 produced with the task VPLOT. The dots are the data while the solid line is the model.

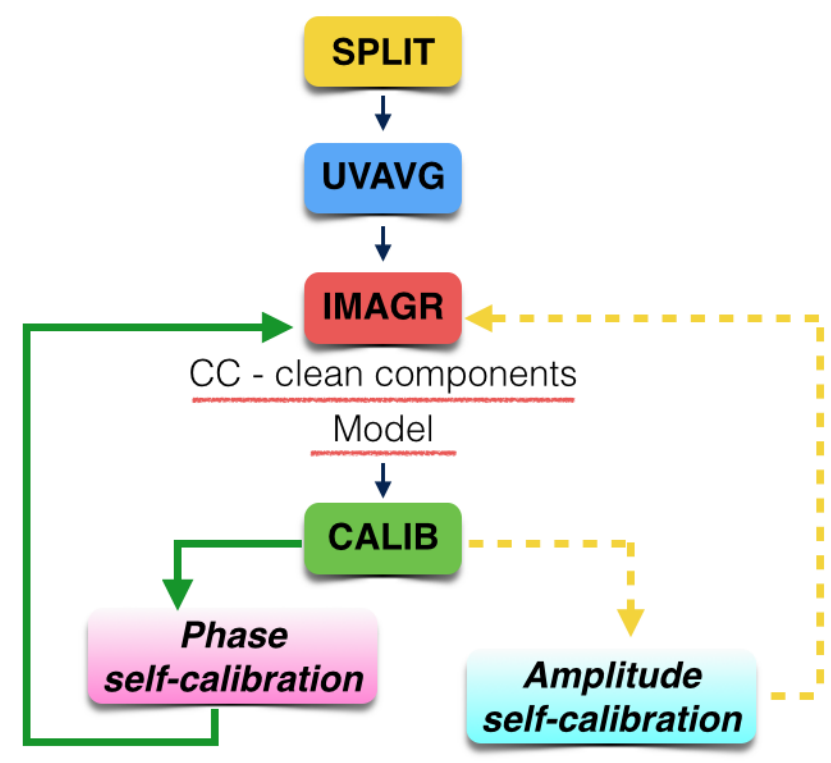

Figure 3.11: A cartoon of the Imaging and self calibration procedure after the split of the data. The phase self calibration (green line) is run cyclically until the convergence, while the amplitude self calibration (yellow dots line) usually is necessary one time at the end of the procedure in order to properly set the gains. 


\section{Chapter 4}

\section{Data Analysis}

In this Chapter the analysis of the two CSO, J1944+5448 and J0111+3906 is described. In Section 5.1 the multifrequency images obtained for each source are shown. Then the main observational parameters achieved from the two CSO are presented. Finally Section 5.3 gives a description of the main morphological features of each source taking into account the global information obtained from the images and the observational parameters.

\subsection{Multifrequency Images}

After the convergence of the self calibration procedure (see Section 3.4.4), I obtained the final images of the two targets J1944+5448 and J0111+3906.

Images were obtained following the standard procedures with the task IMAGR. The final images were cleaned down to the thermal noise and restored with full resolution at each frequency. Contour images were created by means of the task KNTR. The rms noise of each images was measured with the task TVSTAT.

For the spectral index analysis a new set of images (low resolution images) was obtained by considering the visibilities in the $u v$-range 6000-47200 $\mathrm{M} \lambda$ (for the source J0111+3906) and 6000-140550 M $\lambda$ (for the source J1944+5448), in common to the observations at all frequencies. Such images have the same pixel-size (CELLSIZE $=0.1$ mas) and were restored with the same clean beam. We must remark that the uv-coverage does not match as it could happen in VLA observations and this turns into some gradients in the spectral index images in particular in the weak features. The spectral index images were obtained using the low resolution images by means of the task COMB.

Once the low resolution images had been produced, we overlapped them in order to produce the spectral index images. The overlapping can be done only in the case the two images are well aligned, and this is possible by means of the task LGEOM. The alignment was done taking as reference position a point-like or compact component (like one hot spot or the core). One of the images is then shifted and then by means of the task COMB we produced the spectral index distribution image of the radio sources. 
Hereafter I will use the following labels:

- W-HS : West hot spot

- E-HS : East hot spot

- W-L : West lobe

- E-L : East lobe

- NE-HS : North East hot spot

- SW-HS : South West hot spot

- C: core

\subsubsection{J1944+5448}

In this section the images of the source J1944+5448 observed in C and X bands are presented. The off-source rms noise and the beam size (plotted on the bottom left corner of each image) are presented in the caption of each figure.

The spectral index images produced for the source J1944+5448 is between 4.8 and 8.4 GHz.

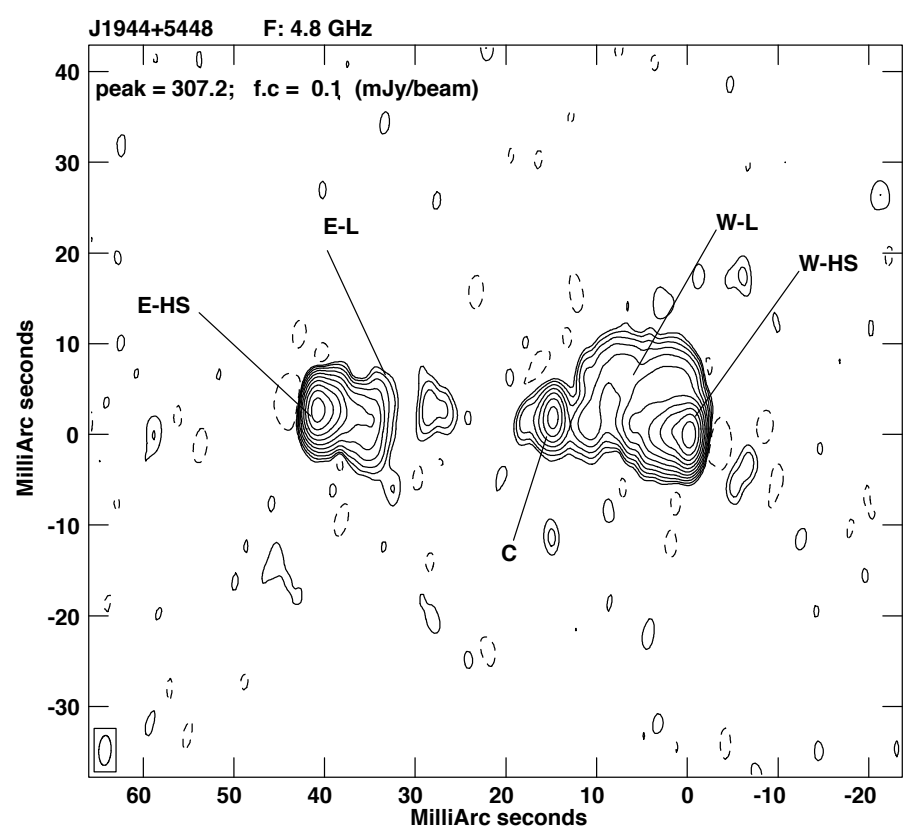

Figure 4.1: Full resolution image in C band for the source J1944+5448. The off-source rms noise is $61 \mu \mathrm{Jy} /$ beam and the restoring beam is $3.30 \times 1.35 \mathrm{mas}^{2}$ in position angle (p.a.) $-2.98^{\circ}$. The first contour level corresponds to three times the off-source rms noise. Contour levels increase by a factor of 2. 


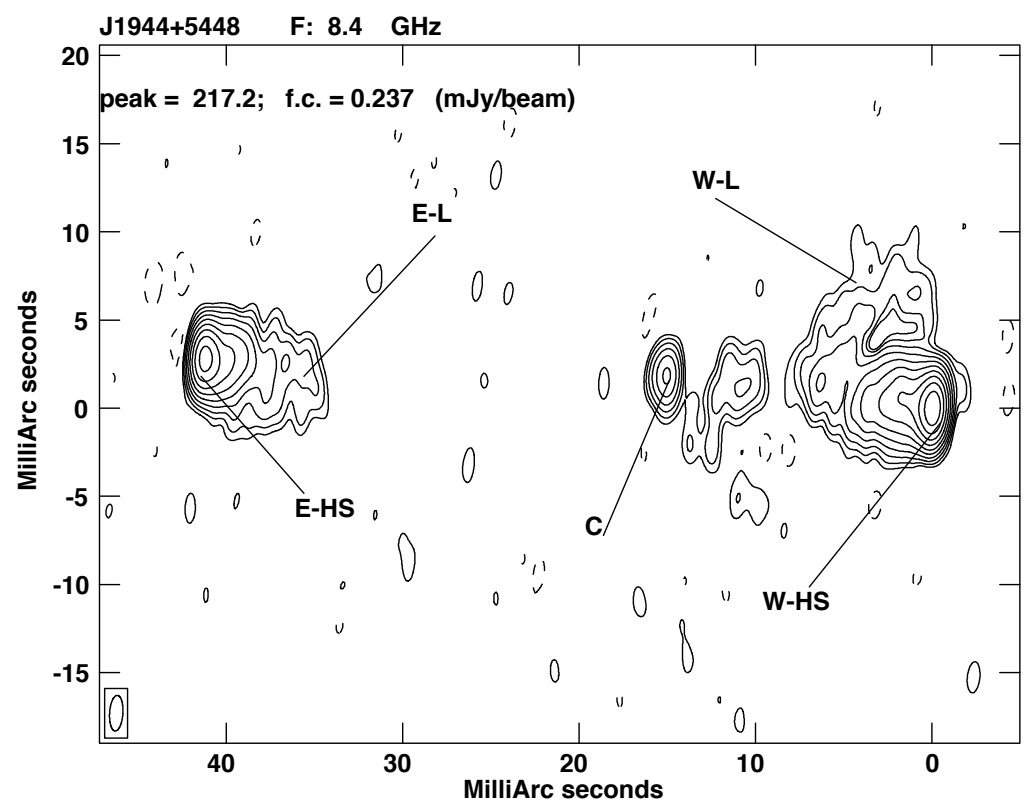

Figure 4.2: Full resolution image of J1944+5448 taken In X band. The off-source rms noise is $70 \mu \mathrm{Jy} /$ beam and the beam size is $2.03 \times 0.75 \mathrm{mas}^{2}$ in p.a. $-3.64^{\circ}$. The first contour level corresponds to three times the off-source rms noise. Contour levels increase by a factor of 2 .

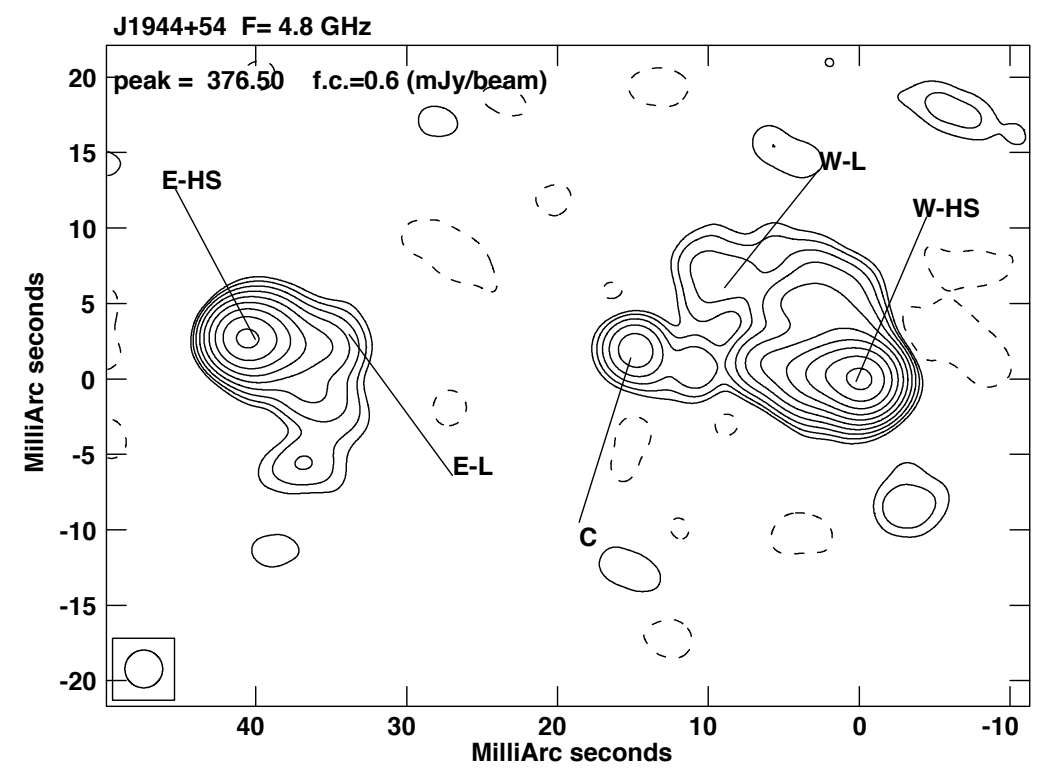

Figure 4.3: Low resolution image of $\mathrm{J} 1944+5448$ in $\mathrm{C}$ band. The beam size is $5 \times 5$ mas $^{2}$ and the off-source rms noise is $235 \mu \mathrm{Jy} / \mathrm{beam}$. The first contour level corresponds to three times the off-source rms noise. Contour levels increase by a factor of 2 . 


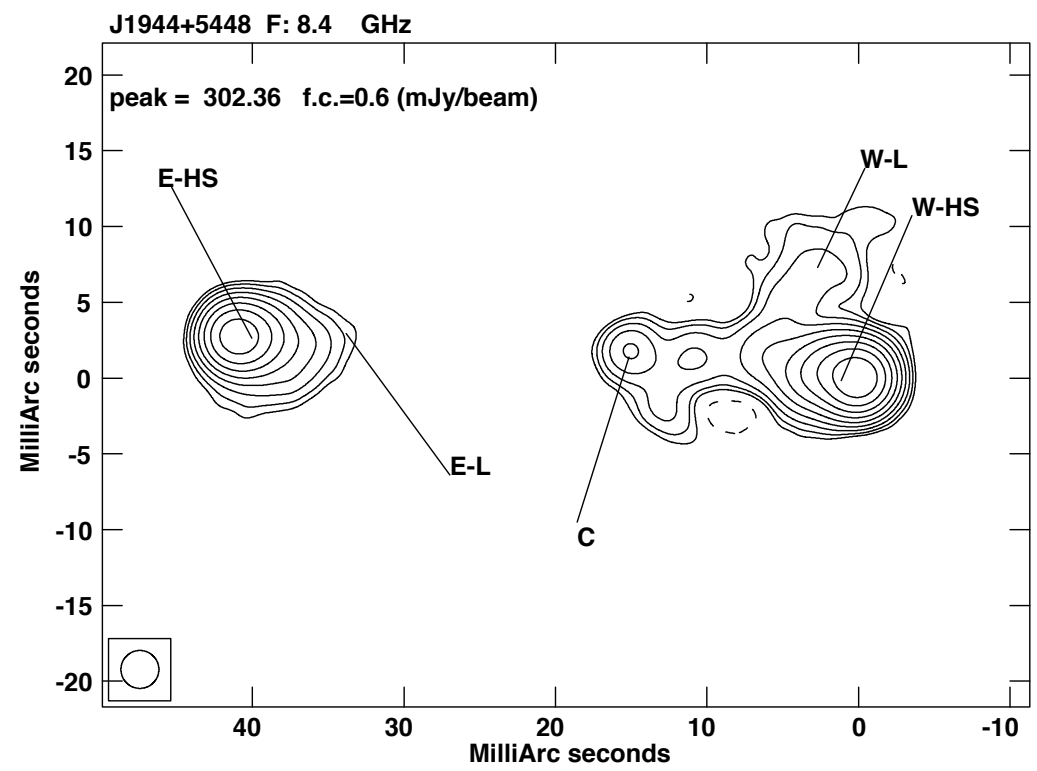

Figure 4.4: Low resolution image of J1944+5448 in X band. The beam size is the same used in the $\mathrm{C}$ band $\left(5 \times 5 \mathrm{mas}^{2}\right)$. The off-source rms noise is $102 \mu \mathrm{Jy} /$ beam. The first contour level corresponds to three times the off-source rms noise. Contour levels increase by a factor of 2 .

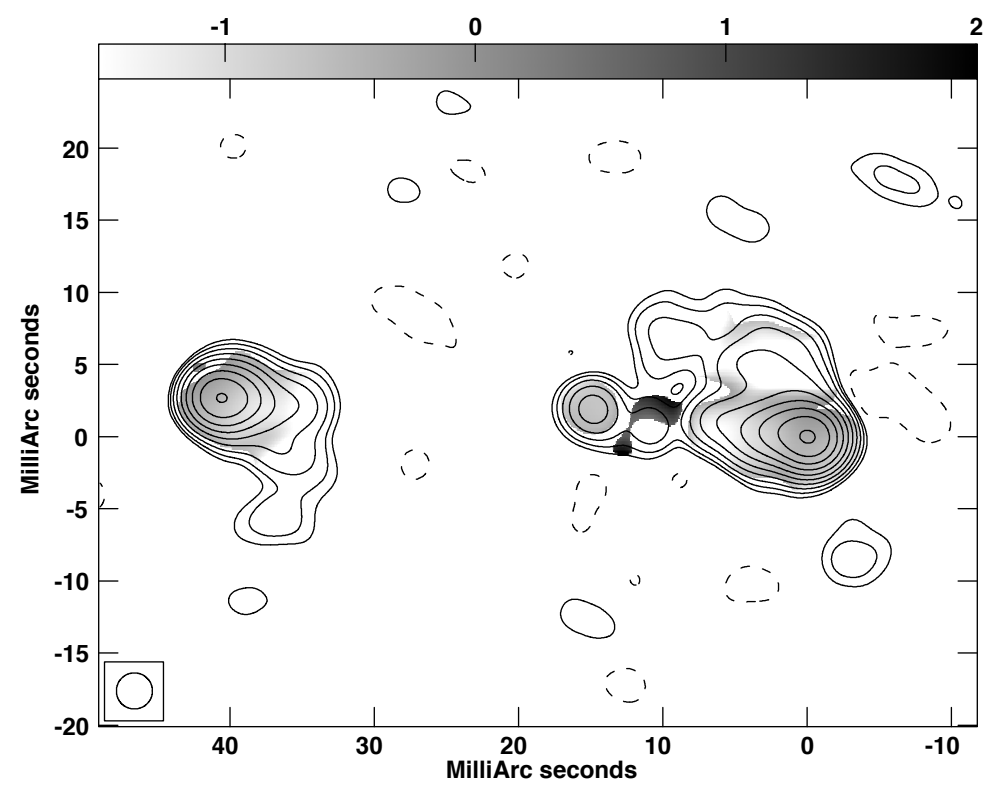

Figure 4.5: Spectral index image between 4.5 and $8.4 \mathrm{GHz}$ of the source J1944+5448. The first contour level corresponds to three times the off-source rms noise. Contour levels increase by a factor of 2 . The contour levels are relative to the low frequency image, while the gray scale is darker toward higher spectral indices. 


\subsubsection{J0111+3906}

The source J0111+3906 was observed in L, C and X bands. In order to improve our knowledge about this source, additional observations taken in $\mathrm{U}$ band $(15.4 \mathrm{GHz})$ from the Mojave Project (Monitoring Of Jets in Active galactic nuclei with VLBA Experiments) ${ }^{1}$ were retrieved and analyzed. In this case the visibilities retrieved from the archive were already calibrated (Lister et al., 2013). Therefore I used them to create the images with the same procedure of cleaning and self calibration described in section 3.4.4. The image in $\mathrm{U}$ band presented in this Chapter refers to observation obtained on 2009, 2 May.

The spectral index images produced for the source J0111+3906 are between 4.8 and 8.4 GHz and 8.4 and $15 \mathrm{GHz}$.

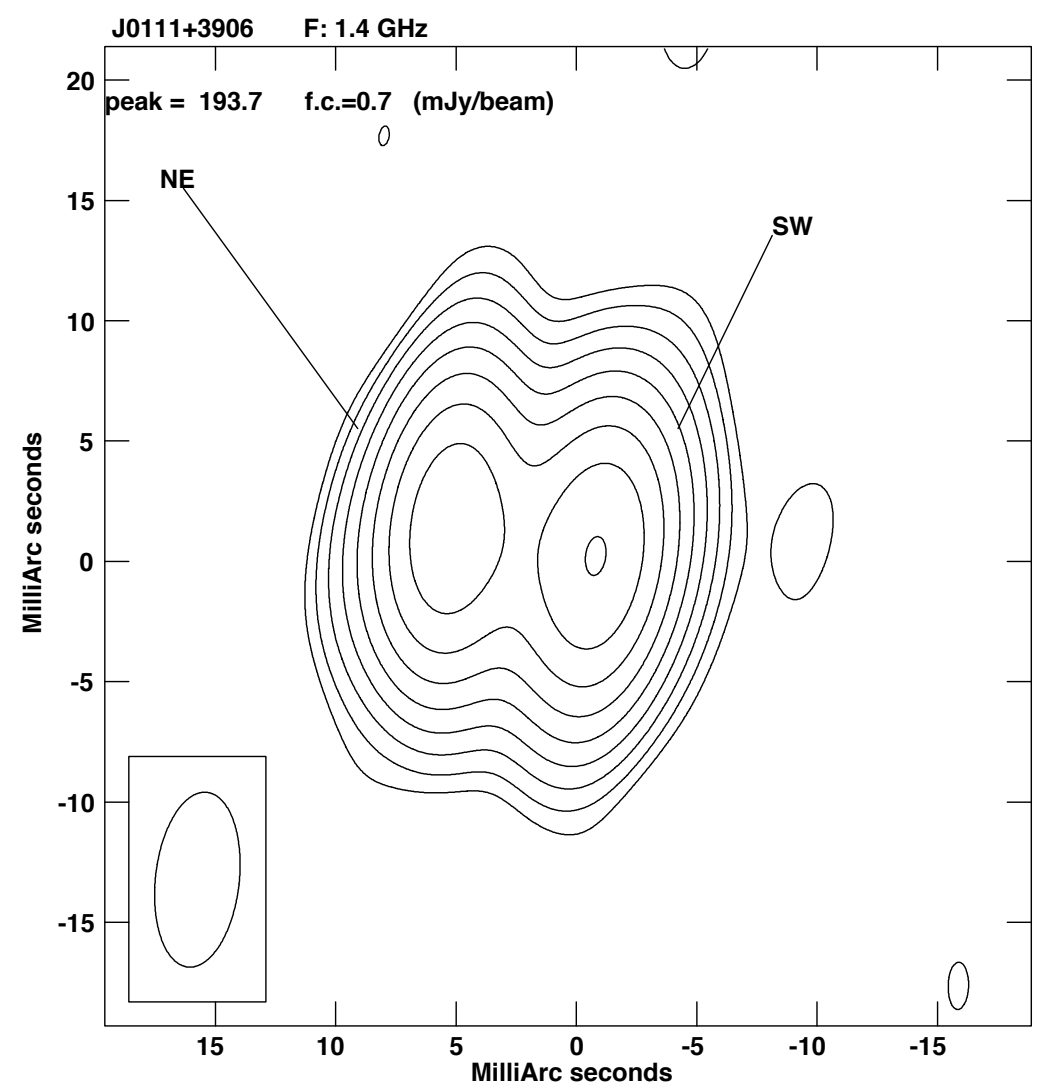

Figure 4.6: Full resolution image in L band of J0111+3906. The off source rms noise is $245 \mu \mathrm{Jy} /$ beam and the beam size is $7.30 \times 3.48 \mathrm{mas}^{2}$ in p.a. $-6.06^{\circ}$. The first contour level corresponds to three times the off-source rms noise. Contour levels increase by a factor of 2 .

\footnotetext{
1 "This research has made use of data from the MOJAVE database that is maintained by the MOJAVE
} team (Lister et al., 2009, AJ, 137, 3718)" 


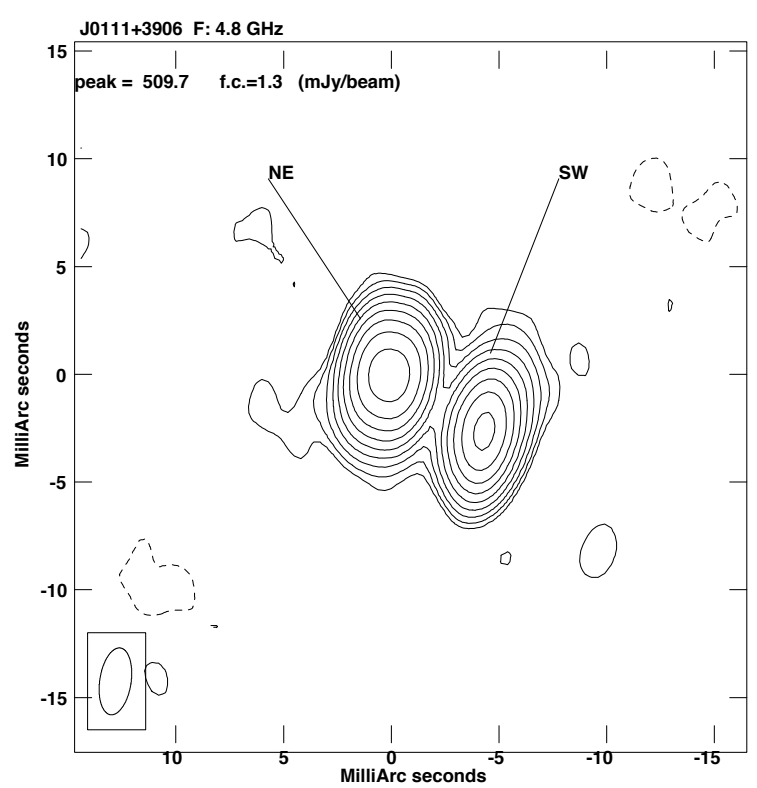

Figure 4.7: Full resolution image in $\mathrm{C}$ band of J0111+3906. The off source rms noise is $149 \mu \mathrm{Jy} /$ beam and the beam size is $3.13 \times 1.45 \mathrm{mas}^{2}$ in p.a. $-8.54^{\circ}$. The first contour level corresponds to three times the off-source rms noise. Contour levels increase by a factor of 2 .

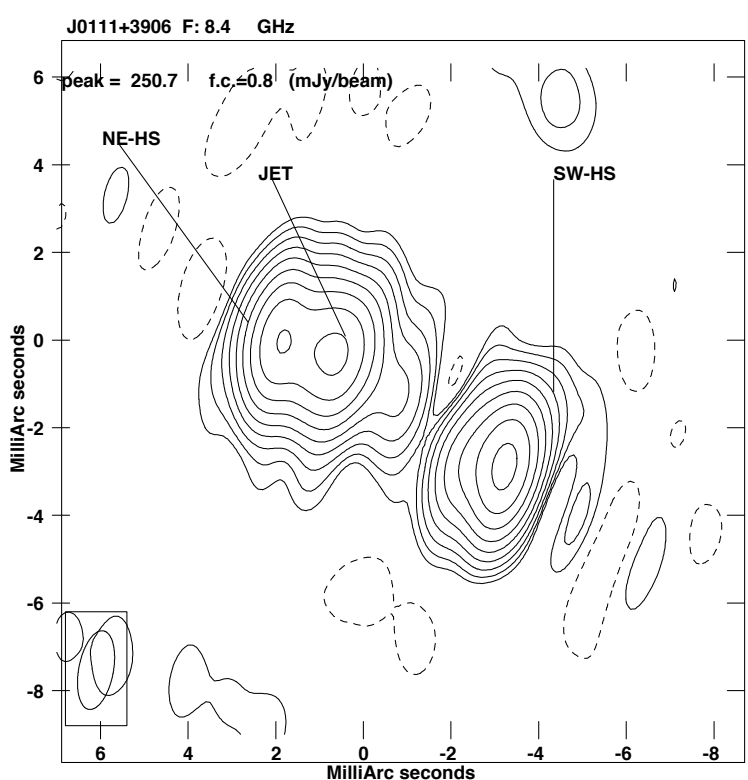

Figure 4.8: Full resolution image in $\mathrm{X}$ band of J0111+3906. The off source rms noise is $279 \mu \mathrm{Jy} /$ beam and the beam size is $1.82 \times 0.79$ mas $^{2}$ p.a. $-10.65^{\circ}$. The first contour level corresponds to three times the off-source rms noise. Contour levels increase by a factor of 2 . 


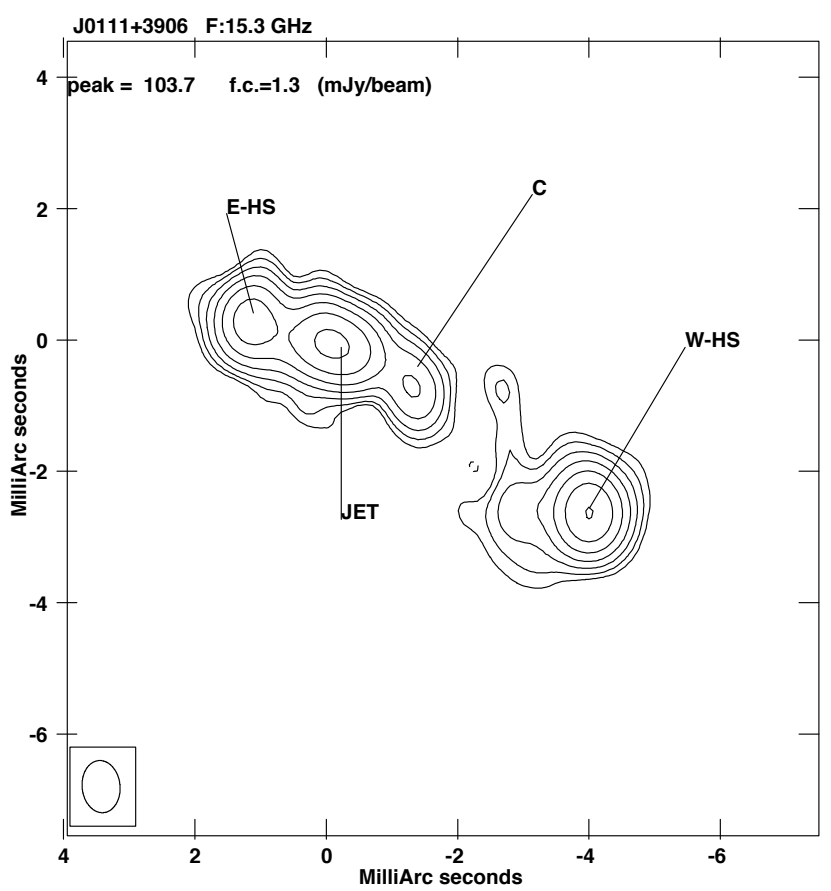

Figure 4.9: Full resolution image in U band of J0111+3906. The off source rms noise is $166 \mu \mathrm{Jy} /$ beam and the beam size is $0.8 \times 0.5 \mathrm{mas}^{2}$ in p.a. $5.82^{\circ}$. The first contour level corresponds to three times the off-source rms noise. Contour levels increase by a factor of 2 .

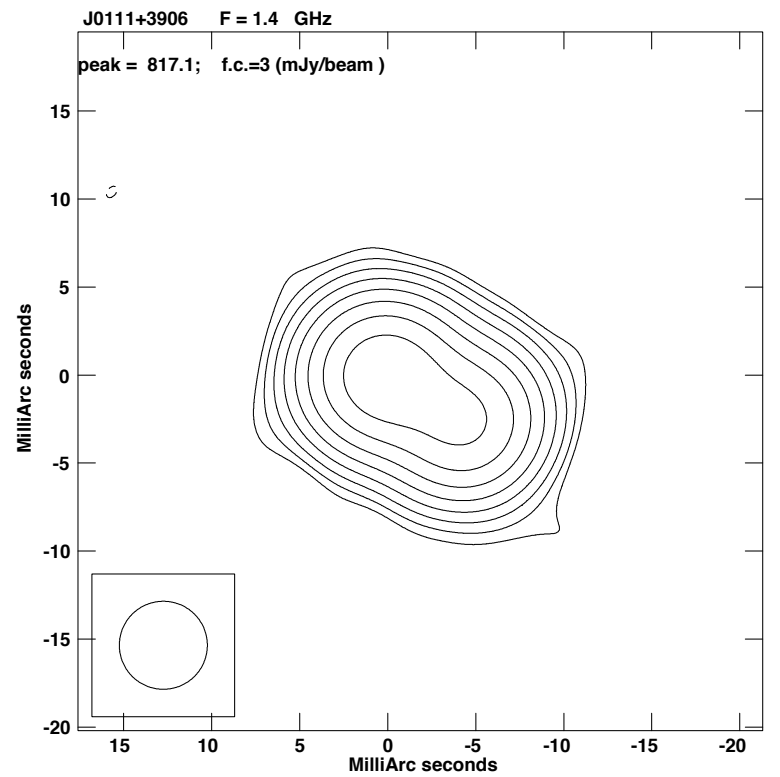

Figure 4.10: Low resolution image of J0111+3906 in C band. The beam size $5 \times 5$ mas $^{2}$. The off source rms noise is $1.143 \mathrm{mJy} /$ beam. The first contour level corresponds to three times the off-source rms noise. Contour levels increase by a factor of 2 . 


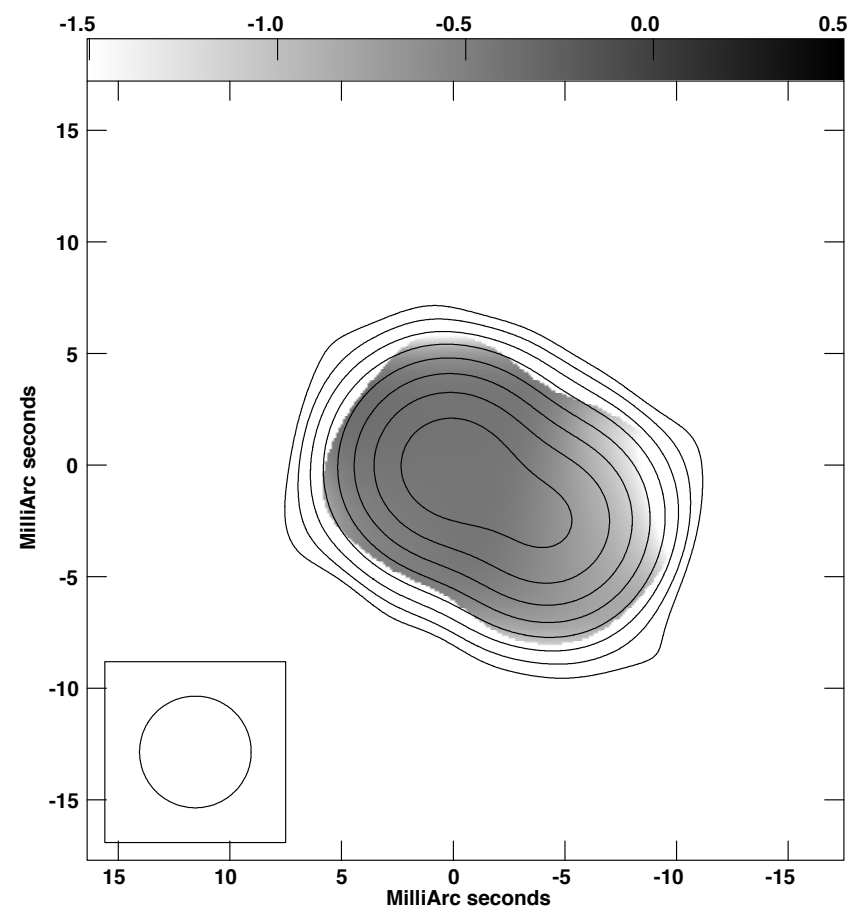

Figure 4.11: Spectral index image between 4.8 and $8.4 \mathrm{GHz}$ for J0111+3906. The first contour level corresponds to three times the off-source rms noise. Contour levels increase by a factor of 2 . The contour levels are relative to the high frequency image, while the gray scale is darker toward higher spectral indices.

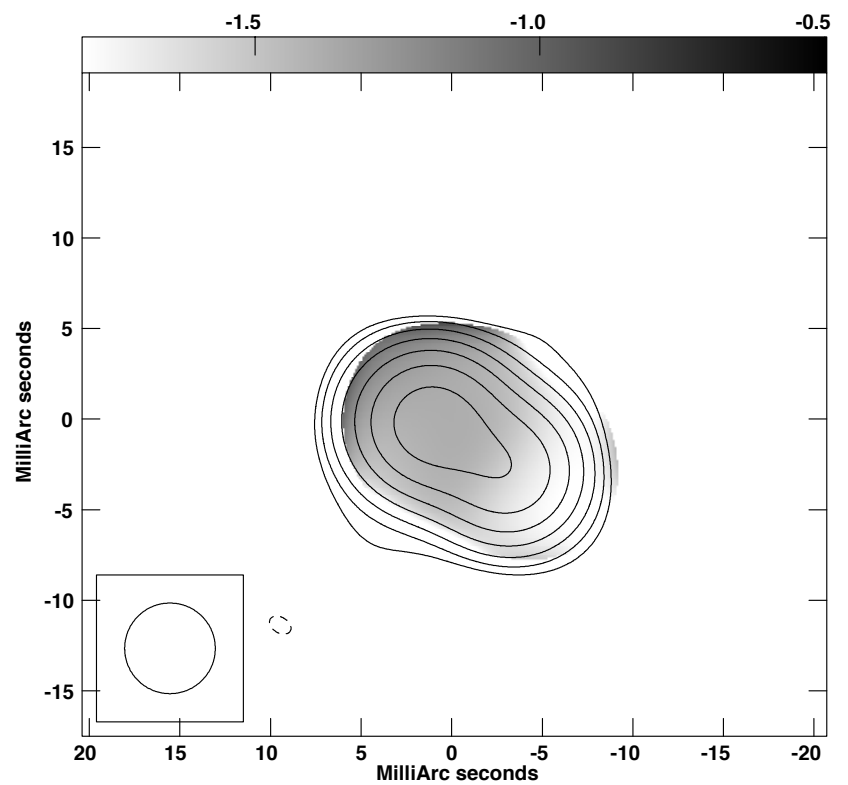

Figure 4.12: Spectral index image between 8.4 and $15 \mathrm{GHz}$ for J0111+3906. The first contour level corresponds to three times the off-source rms noise. Contour levels increase by a factor of 2 . The contour levels are relative to the high frequency image, while the gray scale is darker toward higher spectral indices. 


\subsection{Observational Parameters}

I derived the main observational parameters of J1944+5448 and J0111+3906 considering the images presented in the previous Section and using two main tasks in AIPS:

- INPFIT: it is a procedure to select the area and the peak position of the components which will be considered as a starting model of a Gaussian fit to the image.

- JMFIT: it is a task performing a 2D Gaussian fit of the components on the image plane. It gives in output the peak flux density, the integrated flux density, the de-convolved size, the position angle, and the position of the peak.

JMFIT is a good instrument when considering compact components (as the hot spots and the core), while in the case of diffuse structures, as the lobes, whose size is often larger than the beam, a Gaussian fit may not be adequate. In this case, I used the task TVSTAT to determine the flux density and I measured the size of the diffuse components directly on the images. All the observational parameters measured for each source are presented in Table 4.2.1 and Table 4.2.2.

In the cases in which I obtained the parameters using JMFIT, the full width half maximum of the gaussian fit corresponds to roughly half of the size of the component. Therefore the correction applied is:

$$
\theta_{\mathrm{k}}=1.8 \theta_{\mathrm{FWHM}}
$$

where $\theta_{\mathrm{FWHM}}$ is the measure founded by JMFIT.

\section{Errors}

The flux density error $\sigma_{\mathrm{S}}$ has the contribution from two terms. The first is associated to the rms of the image and is defined as:

$$
\sigma_{\mathrm{rms}}=\mathrm{rms} \times \sqrt{\mathrm{N}_{\mathrm{B}}}
$$

where $\mathrm{N}_{\mathrm{B}}$ is the ratio between the area of the source considered and the area of the beam. The second term $\sigma_{\mathrm{c}}$ is the uncertainty of the absolute flux density scale obtained during the calibration process. We assume that the uncertainty is the scatter observed in the gain curve (Fig.3.6, top panel) in each frequency band and is about $\sim 5 \%, \sim 10 \%$ and $\sim 12 \%$ for $\mathrm{L}, \mathrm{C}$ and $\mathrm{X}$ band respectively, while in $\mathrm{U}$ band is $\sim 5 \%$ as reported in Lister et al., (2003). 
Therefore the total flux density error is:

$$
\sigma_{\mathrm{S}}=\sqrt{\sigma_{\mathrm{rms}}^{2}+\sigma_{\mathrm{c}}^{2}}
$$

In our images the rms is usually about $\sim 10^{-4}, 10^{-5} \mathrm{Jy} /$ beam and is negligible for compact components. In case of extended low-surface brightness components like the lobes, the error on the flux density is the combination of the two contribution. Therefore the error on the flux density is mainly due to the calibration processes at least for the brightest components.

Since JMFIT provides the position of each gaussian component, we estimate the error on that position by means of

$$
\Delta \mathrm{r}=\frac{\mathrm{a}}{\mathrm{S}_{\mathrm{p}} / \mathrm{rms}}
$$

where a is the component de-convolved major-axis, $\mathrm{S}_{\mathrm{p}}$ is its peak flux density and rms is the $1 \sigma$ noise level measured on the image plane (Polatidis \& Conway 2003). In the case the error found with Eq.4.3 is unreliably small, we assume a more conservative value for $\Delta \mathrm{r}$ as the $10 \%$ of the minor axis of the beam (Orienti et al., 2011).

\subsubsection{J1944+5448}

Full resolution images of J1944+5448 show three main compact components (two hot spot and the core) and two diffuse structures (the lobes). Hereafter they will be termed according to Fig. 4.1.

The results obtained for the source $\mathrm{J} 1944+5448$ are reported in Table 4.1

\begin{tabular}{lccccc}
\hline \multicolumn{5}{c}{$\mathbf{J 1 9 4 4 + 5 4 4 8}$} \\
\hline & $\mathrm{S}_{\mathrm{C}}(\mathrm{mJy})$ & $\mathrm{S}_{\mathrm{X}}(\mathrm{mJy})$ & $\theta_{1}(\mathrm{mas})$ & $\theta_{2}$ (mas) & p.a. (degree) \\
\cline { 2 - 6 }$\underline{\mathrm{W}-\mathrm{HS}}$ & $452 \pm 43$ & $314 \pm 31$ & 0.783 & 0.723 & 62.7 \\
$\underline{\text { E-HS }}$ & $234 \pm 22$ & $135 \pm 15$ & 0.956 & 0.688 & 81.5 \\
$\underline{\text { Core }}$ & $21 \pm 3$ & $12 \pm 2$ & 0.756 & 0.651 & 56.8 \\
$\underline{\text { W-Lobe }}$ & $157 \pm 15$ & $146 \pm 14$ & 1.505 & 1.347 & 90 \\
$\underline{\text { E-Lobe }}$ & $38 \pm 2$ & $32 \pm 3$ & 1.241 & 0.689 & 108 \\
\hline
\end{tabular}

Table 4.1: Observational parameters of J1944+5448. Column 1: source component,Column 2: flux density in $\mathrm{C}$ band in mJy, Column 3 : flux density in X band in mJy, Column 5: major axis in mas, Column 4: minor axis in mas, Column 5: position angle of the major axis in degree. 


\subsubsection{J0111+3906}

Full resolution images of J0111+3906, (Fig.4.9) show three main compact components (two hot spots and a jet in the North-East direction). Hereafter they will be termed according to Fig.4.9. The observational parameters of J0111+3906 are presented in Table 4.2. Since the radio source is partially unresolved in L and $\mathrm{C}$ bands (Fig.4.6 and Fig4.7) $\mathrm{I}$ report only the parameters obtained at high frequencies ( $\mathrm{X}$ and $\mathrm{U}$ band). In addition only the flux density at $\mathrm{U}$ band of the core is reported because at lower frequencies it is indistinguishable from the emission of the other components.

\begin{tabular}{|c|c|c|c|c|c|}
\hline \multicolumn{6}{|c|}{$\mathrm{J} 0111+3906$} \\
\hline & $\mathrm{S}_{\mathrm{X}}(\mathrm{mJy})$ & $\mathrm{S}_{\mathrm{U}}(\mathrm{mJy})$ & $\theta_{1}$ (mas) & $\theta_{2}$ (mas) & p.a. (degree) \\
\hline SW-HS & $352 \pm 35$ & $114 \pm 11$ & 0.766 & 0.535 & 93 \\
\hline NE-HS & $246 \pm 30$ & $93 \pm 10$ & 1.326 & 0.498 & 80.2 \\
\hline Core & - & $31 \pm 3$ & 0.798 & 0.781 & 45 \\
\hline Jet & $468 \pm 43$ & $189 \pm 20$ & 1.350 & 0.239 & 77.1 \\
\hline
\end{tabular}

Table 4.2: Observational parameters of J0111+3906. Column 1: source component, Column 2: flux density in $\mathrm{C}$ band in mJy, Column 3 : flux density in $\mathrm{X}$ band in mJy, Column 5: major axis in mas, Column 4: minor axis in mas, Column 5 : position angle of the major axis in degree.

Since in $\mathrm{L}$ and $\mathrm{C}$ bands the resolution is not enough for discriminating the various source sub components in Table 4.3 we provide the observational parameters of two regions of the source J0111+3906, according to Fig.4.13: the North-East region and the SouthWest region (hereafter $\mathrm{N}-\mathrm{E}$ and $\mathrm{S}-\mathrm{W}$ regions respectively). The former is composed by the jet, the N-E hot spot and the core while the latter comprises the S-W hot spot and the weak lobe. In order to avoid further misidentification of the components we made use of the low resolution images (see for example Fig. 4.10) in L, C, X and U bands of J0111+3906.

The results obtained considering this approach are presented in Table 4.3. 


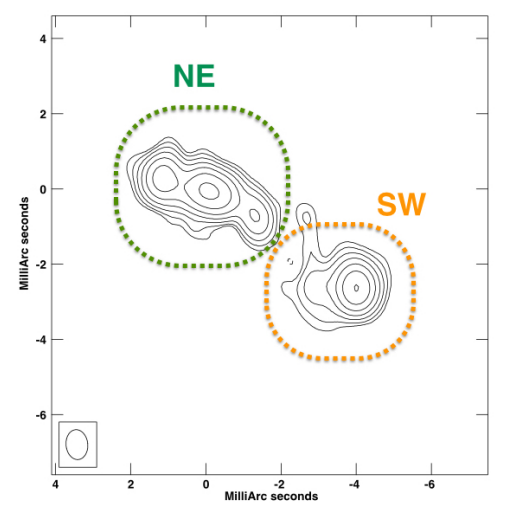

Figure 4.13: The regions of J0111+3906 considered in this work for the data analysis.

\begin{tabular}{lccccccc}
\hline & \multicolumn{7}{c}{ J0111+3906 } \\
\hline & $\begin{array}{c}\mathrm{S}_{\mathrm{L}} \\
(\mathrm{mJy})\end{array}$ & $\begin{array}{c}\mathrm{S}_{\mathrm{C}} \\
(\mathrm{mJy})\end{array}$ & $\begin{array}{c}\mathrm{S}_{\mathrm{X}} \\
(\mathrm{mJy})\end{array}$ & $\begin{array}{c}\mathrm{S}_{\mathrm{U}} \\
(\mathrm{mJy})\end{array}$ & $\begin{array}{c}\theta_{1} \\
(\mathrm{mas})\end{array}$ & $\begin{array}{c}\theta_{2} \\
(\mathrm{mas})\end{array}$ & $\begin{array}{c}\text { p.a. } \\
(\text { degree })\end{array}$ \\
\cline { 2 - 8 } & $248 \pm 19$ & $828 \pm 66$ & $370 \pm 37$ & $228 \pm 12$ & 1.782 & 0.700 & 79 \\
$\underline{\text { North-East region }}$ & $260 \pm 26$ & $529 \pm 53$ & $368 \pm 36$ & $199 \pm 10$ & 1.292 & 0.746 & 85 \\
\hline South-West region & 260 & & & & & \\
\hline
\end{tabular}

Table 4.3: Observational parameters of the two regions of J0111+3906. Column 1: source component, Column 2: flux density in C band in mJy, Column 3 : flux density in X band in mJy, Column 5: major axis in mas, Column 4 : minor axis in mas, Column 5 : position angle of the major axis in degree. 


\subsection{Morphological Description}

The radio images and the observational parameters obtained for the two CSO give us many pieces of information about the morphological features of these radio sources. A detailed description of each source is provides in the following Sections.

\subsubsection{J1944+5448}

The source J1944+5448 has a total angular size of $(41.2 \pm 0.1)$ mas corresponding to a linear size $\mathrm{LS}=168.6 \pm 0.4 \mathrm{pc}$ at the redshift of the host galaxy (see table 3.4 ).

Considering Fig.4.1 (4.5 GHz) the source structure is dominated by two bright components, two hot spots, at the opposite end of the radio source. Both components are resolved and have inward tails similar to lobes. The western component is the brightest one with a total flux density ratio $\mathrm{R}_{\mathrm{s}} \sim 3$ with respect to the eastern hot spot. Between the two hot spots there is an unresolved component which may host the source core, whose position is slightly closer to the brightest component. The radio structure is reminiscent of an FRII radio galaxy and the classification as CSO by (Phillips \& Mutel, 1982) can be supported.

The $\mathrm{X}$ band image (Fig. 4.2) confirms what is visible in Fig. 4.1. The core is clearly detected while the diffuse emission becomes fainter, that is a clear indication of a steep spectral index (Fig. 4.5). In addition the position angle in $\mathrm{C}$ is consistent with that in $\mathrm{X}$ band indicating that the source is not self absorbed in this frequency range, implying that in these bands the spectrum of the source J1944+5448 is optically thin.

The low resolution images in $\mathrm{C}$ and $\mathrm{X}$ band (Fig. 4.3 and Fig. 4.4 respectively) have off-source rms noise $\gg 100 \mu \mathrm{Jy} /$ beam. The three main compact components are visible even if the core is fainter (as for the full resolution images) at higher frequency. In addition the structure of the lobes, in particular the West one, are less defined in X band. In fact, the lobes are weak diffuse radio components with a steep spectrum as pointed out in the spectral index distribution images of the lobes (Fig. 4.5). In particular, the western lobe has $\alpha_{4.8}^{8.4} \sim-1.4$ while the eastern one has $\alpha_{4.8}^{8.4} \sim-1.8$. This agrees with the ageing of the electron population that fills the lobes.

From the spectral index image between the $\mathrm{C}$ and $\mathrm{X}$ bands (Fig. 4.5) we notice that the two hot spots have a typical spectral index of about $\alpha \sim-0.7$, while the core has a surprisingly steep spectral index $\alpha \sim-1.2$. This is due to the contamination by the steep spectrum jet base. In addition, there are some gradients and features in the spectral index image that are caused by the complex morphology of J1944+5448. In this case it is difficult to reach a perfect alignment between the two frequency images and this may 
create the observed spectral index structures. Between 4.5 and $8.4 \mathrm{GHz}$ all the spectral indices found are negative, indicating that the spectra of all regions are relative to the optically thin part of the spectrum.

According to the classification by Fanti et al., (1990) the source J1944+5448 is a double/triple source in which two bright components dominate the radio emission.

In the full resolution images, the hot spots are rather compact with an ellipsoidal shape. The lobes are diffuse structures with a complex shape surrounding the hot spots and in particular the western lobe is more extended than the eastern one. Both the lobes are brighter at lower frequencies. This aspect is in agreement with the spectral index distribution of this source.

Considering the flux density ratio $\mathrm{R}_{\mathrm{S}}$ between the components of J1944+5448 we can notice that the W-HS is the brightest one both in $\mathrm{C}$ and $\mathrm{X}$ band. The W-HS is about three times brighter than the E-HS and four time brighter than its corresponding lobe (E-lobe). Moreover the W-HS is also twenty times brighter than the core which is in this case the weakest component in both $\mathrm{C}$ and $\mathrm{X}$ bands.

It is worth noting that the brightest component, the West hot spot, is also the closest to the core. The separation between the W-HS and the core is $\mathrm{d}_{\mathrm{C}-\mathrm{W}} \sim 60 \mathrm{pc}$ while the separation between the E-HS and the core is $\mathrm{d}_{\mathrm{E}-\mathrm{C}} \sim 110 \mathrm{pc}$.

Therefore, the arm length ratio $\mathrm{R}_{\mathrm{r}}$ is:

$$
\mathrm{R}_{\mathrm{r}}=\frac{\mathrm{d}_{\mathrm{E}-\mathrm{C}}}{\mathrm{d}_{\mathrm{C}-\mathrm{W}}} \sim 1.8
$$

This means that J1944+5448 is an asymmetric source where the west hot spot, which is also the brighter one, is the closer to the core (Fig. 4.14). The asymmetric shape of this source is an important feature which will be analyzed in more detail in the next Chapter (Section 5.4).

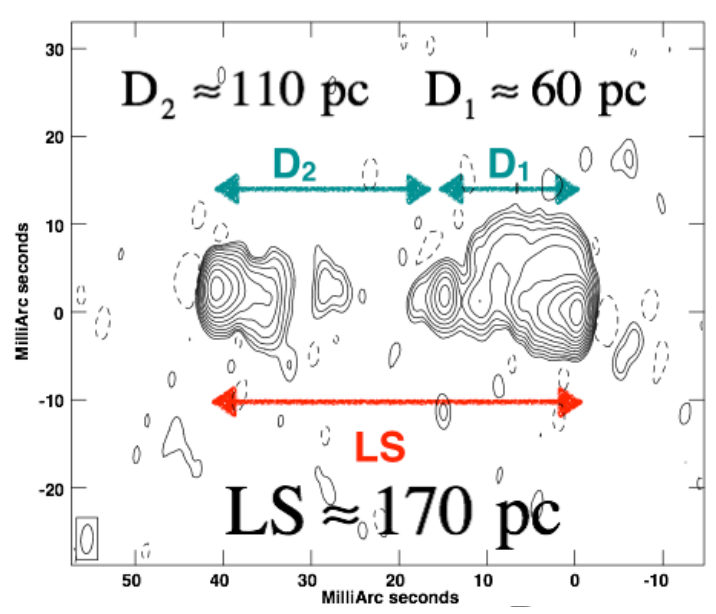

Figure 4.14: J1944+5448 


\subsubsection{J0111+3906}

At low frequencies, (Fig. 4.6 and Fig. 4.7), the source is marginally resolved into two substructures. There is a difference in the position angle between the image in $\mathrm{L}$ and those at higher frequencies $\left(\sim-9^{\circ}\right.$ and $\sim-6^{\circ}$ respectively). This is probably due to the fact that the source is self-absorbed at low frequency. This is also confirmed by the spectral index study between the $\mathrm{L}$ and $\mathrm{C}$ band. Between these two frequencies the spectral index varies between $0.9<\alpha_{\text {thick }}<1.19$ and $0.8<\alpha_{\text {thick }}<1.15$ in the two substructures (NE and SW respectively).

In $\mathrm{X}$ band the source components are not clearly resolved and we see only three large sub-structures of the source, one in particular (in the North-East direction) dominating the whole flux density. This bright component has a flux density of about $\sim 470 \mathrm{mJy}$ that is roughly the $46 \%$ of the total source flux density which is about $900 \mathrm{mJy}$.

On the contrary, image in U band clearly shows several sub-structures of J0111+3906. In this case the two hot spots are well resolved in the North-East and South-West direction, respectively. The core is visible in between the two hot spots. It is a weak component accounting for $\sim 7 \%$ of the total flux density at $15 \mathrm{GHz}$. From the image in $\mathrm{U}$ band we see that the bright region observed in $\mathrm{X}$ band, is a bright jet which elongates in the North-East direction. The jet component accounts for $\sim 46 \%$ and $\sim 50 \%$ of the total flux density of the source in $\mathrm{X}$ and $\mathrm{U}$ bands, respectively. Moreover hints of a weak lobe is present in the $\mathrm{S}-\mathrm{W}$ region.

The reason why the high frequency and thus the high resolution image provides a better description of the components of this source is due to the fact that J0111+3906 is a very compact source. It has an angular size of ( $5.4 \pm 0.8$ mas) corresponding to $\mathrm{LS}=(41.2 \pm 0.6) \mathrm{pc}$ at the redshift of the host galaxy. Therefore, in this case the resolution is a fundamental factor in order to study its morphology and to detect and resolve the sub-structures of the radio source.

By means of the $\mathrm{U}$ band image we can also derive the hot spot separation with respect to the core which is $\mathrm{d}_{\mathrm{C}-\mathrm{SW}} \sim 26.7 \mathrm{pc}$ for the South-West hot spot and $\mathrm{d}_{\mathrm{NE}-\mathrm{C}} \sim 14.5 \mathrm{pc}$ for the North East one. J0111+3906 is an asymmetric source and in this case North-East hot spot, which is also the brighter one, is the closer one to the core .

In J0111+3906 the hot spots are compact with a roughly spherical shape as the core. The jet has a cylindrical/elliptical elongated morphology and it seems to be not so collimated as we expect. The N-E hot spot is well aligned with the jet direction, both with a position angle of about $\sim 78^{\circ}$.

According to the classification by Fanti et al (1990) we may classify this object as a double/triple source. The shape of J0111+3906 is reminiscent of the typical FRII morphology and confirms the CSO classification. The analysis of the low resolution images indicates that the NE region is the brighter one in all the frequency bands. The NE region has a flux density roughly two times greater than the other at almost all frequencies.

The spectral index image between $\mathrm{C}$ and $\mathrm{X}$ bands shows that the the N-E region, 
which is dominated by the hot spot and the jet, has spectral indices ranging between $\alpha \sim-0.5$ and $\alpha \sim-0.7$. On the other hand, the $\mathrm{S}-\mathrm{W}$ region is steeper with the spectral index that ranges between $\alpha \sim-0.9$ and $\alpha \sim-1.2$. In this case, the steepening is likely due to the presence of the lobe (S-W lobe). In fact, jets and hot spots are both refreshed with new accelerated particles while the lobes host electrons that are ageing and that are losing energy and for this reason the spectrum is steeper. This is confirmed by the study of the spectral indices between the $\mathrm{X}$ and $\mathrm{U}$ bands (Fig. 4.12). The spectra indices are steeper than in the C-X image (Fig.4.11), with a value ranging between $\alpha \sim-1.1$ and $\alpha \sim-1.8$ from the North East region to the South West. This fact may be due to the steepening that occurs at high frequencies. However, all the negative values of the spectral indices between the $\mathrm{C}, \mathrm{X}$ and $\mathrm{U}$ band indicate that in this frequency range both the source regions (NE and SW) are optically thin. 


\section{Chapter 5}

\section{Discussion}

In Section 5.1 the physical parameters calculated for each source are presented. Then the results obtained from the multi-epoch study of the two CSO are discussed (Section 5.2). In Section 5.3 the results on the estimate of the age of these sources are given. Then the symmetries on the two sources are analyzed (Section 5.4). The description of the spectral features are reported in Section 5.5. Finally the extended emission associated with J0111+3906 is described in Section 5.6.

\subsection{Physical Parameters}

Considering the observational parameters presented in the previous Chapter I computed the physical parameters of each radio source, such as the volume, the energy and the magnetic field.

I estimated the volume $(\mathrm{V})$ of each source component considering an ellipsoidal structure:

$$
\mathrm{V}=\frac{\pi}{6} \mathrm{~d}_{1} \cdot \mathrm{d}_{2}^{2}
$$

where $d_{1}$ is the major axis and $d_{2}$ the minor axis of each component. In particular I assumed that the third dimension of the ellipsoid is equal to the minor axis.

I estimated the integrated Luminosity (L) of each component between $10 \mathrm{MHz}$ and $100 \mathrm{GHz}$ according to Eq. 5.3.

$$
\mathrm{L}=4 \pi \mathrm{D}_{\mathrm{L}}^{2}(1+\mathrm{z})^{(\alpha-1)} \int_{10 \mathrm{MHz}}^{100 \mathrm{GHZ}} \mathrm{S}(\nu) \mathrm{d} \nu
$$

where $\mathrm{D}_{\mathrm{L}}$ is the luminosity distance, $\mathrm{z}$ is the redshift, $\alpha$ is the spectral index and $\mathrm{S}(\nu)$ is the flux density. In this case I assumed $\alpha=-0.7$ which is a typical value for a radio source. The quantity $(1+\mathrm{z})^{(-\alpha+1)}$ is the $K$-correction and takes into consideration the 
frequency shift of the emission between the target rest-frame and the observer frame.

The total energy $E_{\text {tot }}$ is

$$
\mathrm{E}_{\mathrm{tot}}=(1+\mathrm{k})^{4 / 7} \mathrm{c}_{\mathrm{el}}^{4 / 7} \mathrm{~L}^{4 / 7} \mathrm{~V}^{3 / 7}
$$

while the minimum energy density $u_{\min }$ is

$$
\mathrm{u}_{\min }=\frac{\mathrm{E}_{\mathrm{tot}}}{\mathrm{V}}=(1+\mathrm{k})^{4 / 7} \mathrm{c}_{\mathrm{el}}^{4 / 7} \mathrm{~L}^{4 / 7} \mathrm{~V}^{-4 / 7}
$$

The magnetic field was estimated according to the equipartition condition (Pacholczyk, 1970). I obtained the equipartition magnetic field, $\mathrm{H}_{\mathrm{eq}}$ according to Eq.5.3 and Eq.5.4 as follows:

$$
\mathrm{H}_{\mathrm{eq}}=(4.5)^{2 / 7}(1+\mathrm{k})^{2 / 7} \mathrm{c}_{\mathrm{el}}^{2 / 7} \mathrm{~L}^{2 / 7} \mathrm{~V}^{-2 / 7}
$$

In this equation $\mathrm{c}_{\mathrm{el}}$ is a tabulated value and depends on the frequency range and on $\delta$. I this case $\mathrm{I}$ used $c_{\mathrm{el}}=2.7 \times 10^{7}$. In addition I assumed $\mathrm{k}=1$ that means that the energy of the proton is proportional to that of the electrons, $\mathrm{E}_{\mathrm{el}}=\mathrm{E}_{\mathrm{pr}}$.

Finally we derived the equipartition pressure, $\mathrm{p}_{\mathrm{eq}}$, from the radiation and magnetic energy density, which is:

$$
\mathrm{p}_{\mathrm{eq}}=\frac{11}{21} \mathrm{u}_{\mathrm{min}}
$$

A detail description of the estimate of the physical parameters in radio sources, is given in the Appendix A. The physical parameters of the source components were computed by using Eq. 5.1, Eq. 5.2, Eq. 5.5, Eq. 5.3, Eq. 5.4 and Eq.5.6. The results are presented in the following sections. 


\section{$5.1 .1 \quad \mathrm{~J} 1944+5448$}

The results on the physical parameters of the components of J1944+5448 are presented in Table 5.1. In order derive the lobe parameters, the contribution of the corresponding hot spot has been subtracted from the flux density. This approach allows us to obtain physical information on the lobes, minimizing the hot spots contamination.

\begin{tabular}{|c|c|c|c|c|c|c|}
\hline \multicolumn{7}{|c|}{$\mathrm{J} 1944+5448$} \\
\hline & $\begin{array}{c}\mathrm{V} \\
\left(\mathrm{pc}^{3}\right)\end{array}$ & $\begin{array}{l}\mathrm{L} 10^{43} \\
(\mathrm{erg} / \mathrm{s})\end{array}$ & $\begin{array}{c}\mathrm{H}_{\mathrm{eq}} \\
(\mathrm{mG})\end{array}$ & $\begin{array}{l}\mathrm{u}_{\min } 10^{-5} \\
\left(\mathrm{erg} / \mathrm{cm}^{3}\right)\end{array}$ & $\begin{array}{c}\mathrm{E}_{\text {tot }} 10^{53} \\
(\mathrm{erg})\end{array}$ & $\begin{array}{c}\mathrm{p}_{\text {eq }} 10^{-5} \\
\left(\text { dyne } / \mathrm{cm}^{2}\right)\end{array}$ \\
\hline W H-S & 85.6 & 3.6 & 28.6 & 33.3 & 8.3 & 17.3 \\
\hline E H-S & 94.7 & 1.7 & 22.1 & 20.4 & 5.7 & 10.1 \\
\hline Core region & 67.1 & 0.16 & 12.6 & 6.4 & 1.26 & 3.3 \\
\hline W Lobe & $97.910^{3}$ & 1.2 & 2.7 & 0.17 & 90 & 0.15 \\
\hline E Lobe & $21.210^{3}$ & 0.26 & 2.8 & 0.16 & 19 & 0.16 \\
\hline
\end{tabular}

Table 5.1: Physical parameters estimated for the components of J1944+5448. Column 1: source component, Column 2: the volume,Column 3: the integrated luminosity, Column 4: the equipartition magnetic field, Column 5: the minimum energy density, Column 6: the total energy, Column 7: the equipartition pressure.

In this source the core region is the most compact component, whose volume is only $\sim 60 \mathrm{pc}^{3}$ and it has the lowest integrated luminosity. The hot spots are compact structures with a high luminosity of the order of $\mathrm{L}_{\mathrm{hs}} \sim 210^{43} \mathrm{erg} / \mathrm{s}$ and high magnetic field intensities of $\geq 22 \mathrm{mG}$. This is due to the fact that according to Eq. 5.5 the magnetic field is inversely proportional to the volume and directly proportional to the luminosity. On the other hand, the lobes are large and extended structures, with a volume one thousand times larger than that of the hot spots. In particular, the West lobe is more extended and brighter than the East lobe. The large volume of the lobes implies that their total energy is higher than the energy contained in the other source components, while the magnetic field in the lobes is one order of magnitude lower than in the hot spots. It is worth noting that the magnetic field lines are frozen in the matter, and as the matter expands the magnetic field become weaker.

The lobes have also the lower equipartition pressure which is consistent with their 
size, since they are diffuse large emission region. On the contrary, the pressure in the hot spots is larger since they are compact structures.

\section{$5.1 .2 \quad \mathrm{~J} 0111+3906$}

The physical parameters calculated for the source J0111+3906 are presented in Table 5.2. I estimated these parameters only for the two regions NE and SW as discussed in Section 4.2.2.

\begin{tabular}{|c|c|c|c|c|c|c|}
\hline \multicolumn{7}{|c|}{$\mathrm{J} 0111+3906$} \\
\hline & $\begin{array}{c}\mathrm{V} \\
\left(\mathrm{pc}^{3}\right)\end{array}$ & $\begin{array}{l}\mathrm{L} 10^{44} \\
(\mathrm{erg} / \mathrm{s})\end{array}$ & $\begin{array}{c}\mathrm{H}_{\mathrm{eq}} \\
(\mathrm{mG})\end{array}$ & $\begin{array}{l}\mathrm{u}_{\min } 10^{-4} \\
\left(\mathrm{erg} / \mathrm{cm}^{3}\right)\end{array}$ & $\begin{array}{c}\mathrm{E}_{\text {tot }} 10^{54} \\
\quad(\mathrm{erg})\end{array}$ & $\begin{array}{c}\mathrm{p}_{\text {eq }} 10^{-5} \\
\left(\text { dyne } / \mathrm{cm}^{2}\right)\end{array}$ \\
\hline$\underline{\mathrm{N}-\mathrm{E} \text { region }}$ & 402.9 & 5.4 & 38.6 & 59.1 & 7.5 & 33.1 \\
\hline $\mathrm{S}-\mathrm{W}$ region & 214.4 & 3.4 & 40.6 & 70.3 & 4.43 & 36.2 \\
\hline
\end{tabular}

Table 5.2: Physical parameters estimated for the two region of J0111+3906. Column 1: source component, Column 2: the volume,Column 3: the integrated luminosity, Column 4: the equipartition magnetic field, Column 5: the minimum energy density, Column 6: the total energy, Column 7: the equipartition pressure.

In the source J0111+3906 the North-East region is the larger one and it has the high integrated luminosity. This may be due to the bright jet that is contained in that region.

The magnetic fields are high in both the two regions $(\sim 38$ and $\sim 40 \mathrm{mG})$. The equipartition pressure is of the order of $10^{-4}$ dyne $/ \mathrm{cm}^{2}$ and in the case of the compact component, SW, the pressure is a little higher than in the NE region. The total energy is larger in the NE region since it is a factor of $\sim 2$ more extended and has the higher luminosity.

Making a comparison between the two sources we see that J0111+3906 has the higher magnetic field and the higher integrated luminosity values. We remark that this source is the most compact which extends only for about $40 \mathrm{pc}$. The higher luminosity of J0111+3906 is due to its higher redshift, $\mathrm{z}=0.668$. The size of the source and its luminosity are the reason why the magnetic field is so high. 


\subsection{Multi-Epoch observations}

In order to improve our knowledge about the two compact symmetric objects, J1944+5448 and J0111+3906, multi-epoch observations already available were analyzed in addition to those presented in the previous Chapters. This approach allows us to estimate the Hot Spot Separation Speed, $\mathrm{v}_{\mathrm{sep}}$. This parameter is fundamental in understanding the expansion rate of these sources and thus for estimating the kinematic age, as discussed in Chapter 2 (Section 2.7).

The hot spot separation speed can be found by interpolating the hot spot separation derived by multi-epoch observations versus the total linear size (LS) of the source. The errors on the linear size were calculated according to Eq. 4.3. The error associated with the hot spot separation speed has been derived by means of the Minimum Chi Square method $\chi^{2}$. The results obtained for each source are discussed in the following Sections.

\section{$5.2 .1 \quad \mathrm{~J} 1944+5448$}

For the source J1944+5448, data from Polatidis \& Conway (2003) and Xu, Reahdead et al. (1995) have been used. All the data used for J1944+5448 are presented in Table 5.3.

\begin{tabular}{|c|c|c|c|c|}
\hline \multicolumn{5}{|c|}{$\mathrm{J} 1944+5448$} \\
\hline \multicolumn{5}{|c|}{ Multi-Epoch Observations } \\
\hline Epoch & $\underline{\mathrm{LS}}(\mu \mathrm{as})$ & $\underline{\sigma_{\mathrm{r}}(\mu \mathrm{as})}$ & $\underline{\text { Paper }}$ & $\underline{B a n d}$ \\
\hline 1991.7 & 40.96 & 0.10 & $\mathrm{~A}$ & $\mathrm{C}$ \\
\hline 1993.3 & 41.06 & 0.08 & $\mathrm{~B}$ & $X$ \\
\hline 1994.9 & 41.00 & 0.10 & $\mathrm{~B}$ & $\mathrm{C}$ \\
\hline 1995.9 & 40.99 & 0.10 & $\mathrm{~B}$ & $\mathrm{C}$ \\
\hline 1998.1 & 41.24 & 0.08 & $\mathrm{~B}$ & $X$ \\
\hline 2000.9 & 41.18 & 0.10 & $\mathrm{~B}$ & $\mathrm{C}$ \\
\hline 2000.9 & 41.20 & 0.08 & $\mathrm{~B}$ & $X$ \\
\hline 2008.3 & 41.24 & 0.10 & $\mathrm{C}$ & $\mathrm{C}$ \\
\hline 2008.3 & 41.44 & 0.08 & $\mathrm{C}$ & $X$ \\
\hline
\end{tabular}

Table 5.3: Data from the multi-epoch observations of J1944+5448. Column 1: epoch of the observations, Column 2: Linear size measured in $\mu$ as, Column 3: error $\sigma_{r}$ estimated according to Eq.4.3 in $\mu$ as, Column 4: references (A: Xu et al., (1995), B: Polatidis \& Conway (2003), C: this work (Section 3.2)), Column 5: frequency band of the observations. 
From the plot of the linear size versus the epoch of observations we derive the hot spot separation speed (Fig.5.1). In this plot I considered separately the observation in C and $\mathrm{X}$ band.

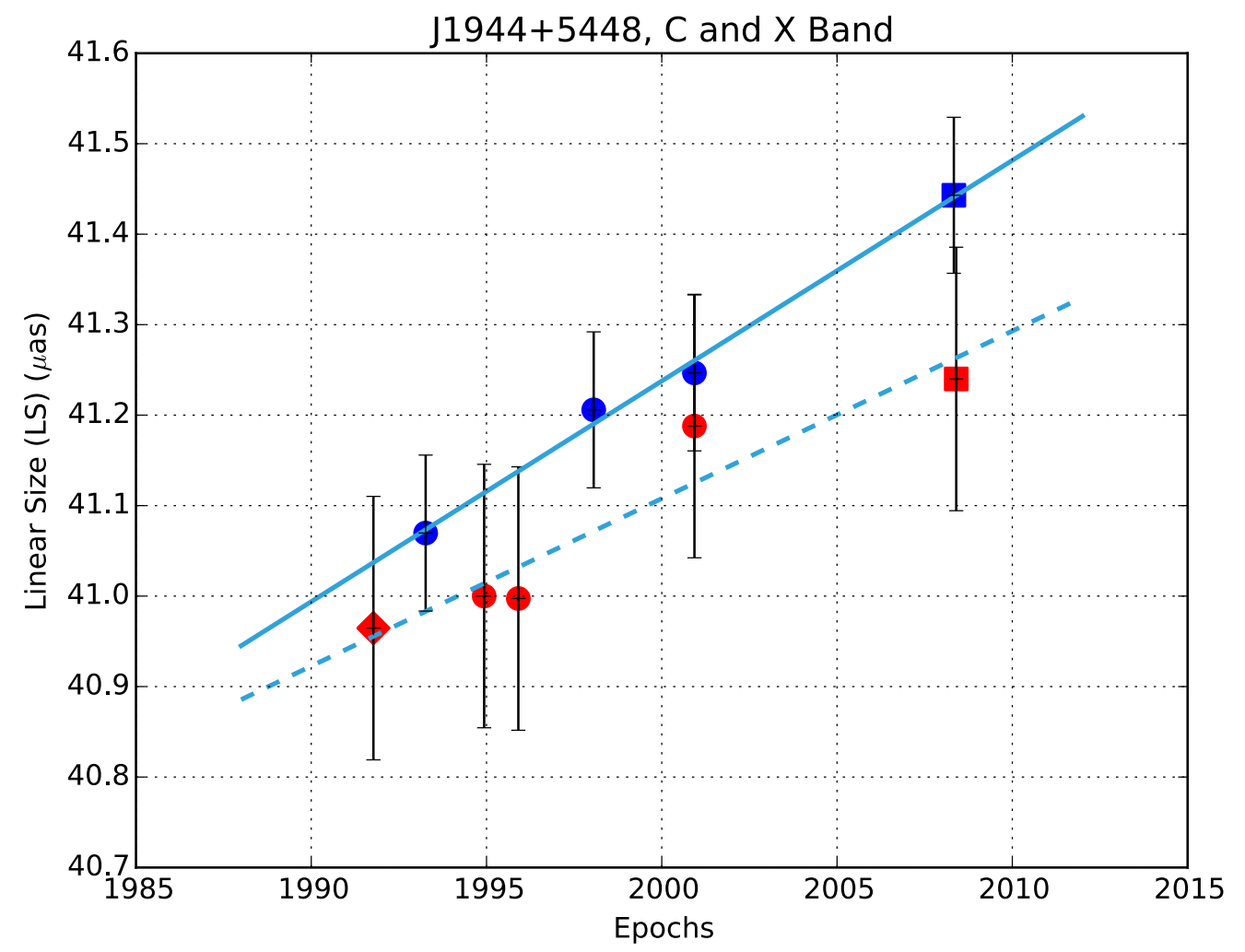

Figure 5.1: Plot of the linear size versus the multi-epoch observations of J1944+5448. The circles represent the data from Polatidis \& Conway (2003), the squares indicate the data of this work (28 April, 2008) and the diamonds refer to the data from Xu et al., (1995). Red simbols represents the observations in C band, blue circle those in X band. 
The light blue line in Fig.5.1 represents the best fit on the data at $8.4 \mathrm{GHz}$, while the dots blue line is the best fit of the data at $5 \mathrm{GHz}$. Th slope is the hot spot separation speed $\mathrm{v}_{\mathrm{sep}}$.

In the case of J1944+5448 ( $\mathrm{X}$ band) I derive:

$$
\mathrm{v}_{\text {sep }}^{\mathrm{X}}=(24.3 \pm 0.7) \mu \text { as } \mathrm{y}^{-1}
$$

which corresponds to

$$
\mathrm{v}_{\mathrm{sep}}^{\mathrm{X}}=(0.25 \pm 0.8) \mathrm{c}
$$

In the case of the $\mathrm{C}$ band, (dots blue line in Fig.5.1) the result is:

$$
\mathrm{v}_{\text {sep }}^{\mathrm{C}}=(18.5 \pm 11.2) \mu \text { as }^{-1}
$$

which corresponds to

$$
\mathrm{v}_{\text {sep }}^{\mathrm{C}}=(0.19 \pm 0.11) \mathrm{c}
$$

These hot spot separation speeds were compared with the one found by Polatidis \& Conway (2003). They estimated $\mathrm{v}_{\mathrm{sep}}^{\mathrm{C}}=(0.26 \pm 0.02) \mathrm{c}$ considering the observations taken in $\mathrm{C}$ band and $\mathrm{v}_{\mathrm{sep}}^{\mathrm{X}}=(0.28 \pm 0.02) \mathrm{c}$ for the $\mathrm{X}$ band observations on the basis of four and three epochs of observations (for C and X band, respectively) from 1991 to 2000. The multi epoch plot of J1944+5448, presented by Polatidis \& Conway (2003) is shown in Fig. 5.2. The results from Polatidis \& Conway (2003) agree with the one found in this work.

The difference between the hot spot separation speed measured using the 4.8 and 8.4 $\mathrm{GHz}$ is related to the fact that at high frequency the resolution is higher. Moreover the source is more optically thin at higher frequencies and this may be related to a better estimation of the linear size and thus of the expansion rate.

The source has expanded of about $\sim 190$ pas in the time range between 2000 and 2008, and also it has expanded of about $\sim 360 \mu a s$ with respect the 1993 observations, and $\sim 430$ pas with respect to the 1991 observation (Xu et al., 1995). Globally the source expanded of about $\approx 1.7 \mathrm{pc}$ over eighteen years. 


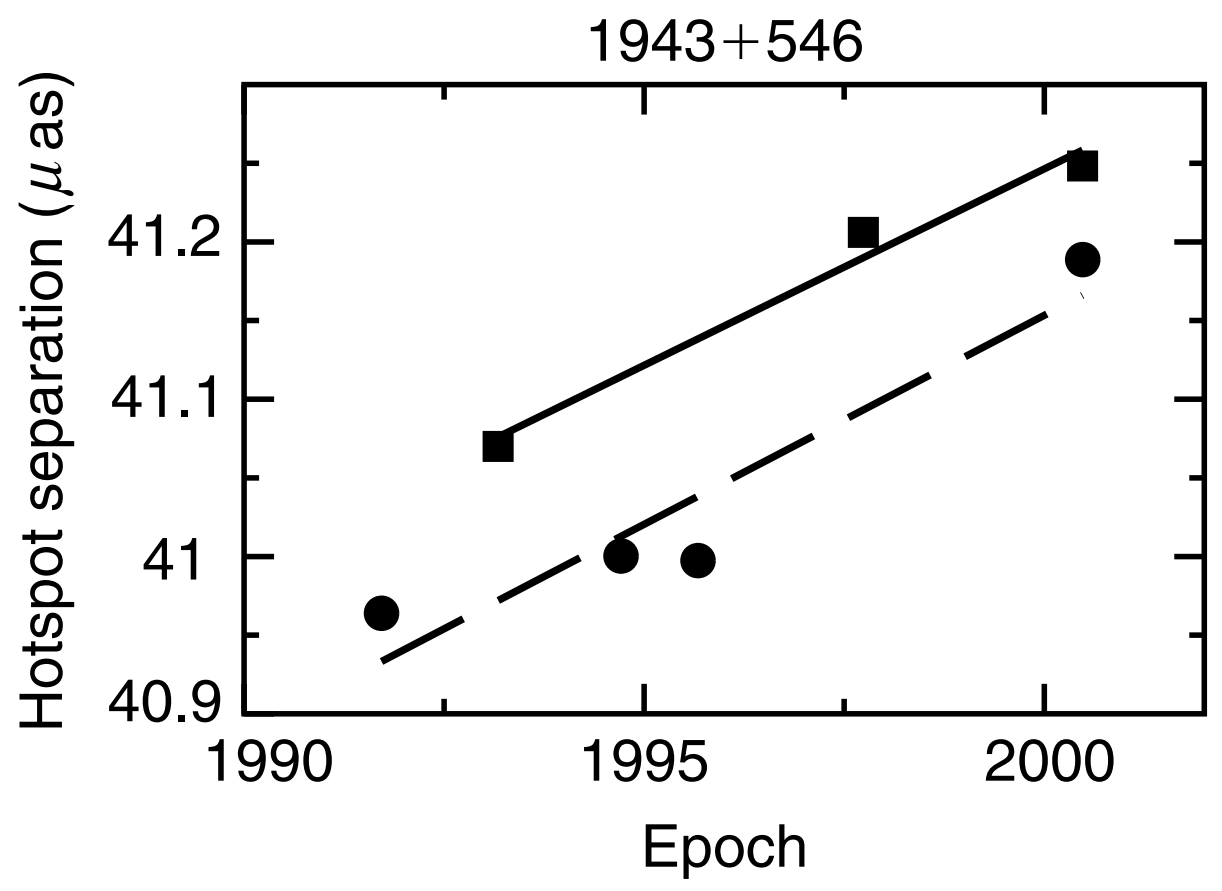

Figure 5.2: The multi-epoch analysis obtained by Polatidis \& Conway (2003). Squares represent the $\mathrm{X}$ band observations, dots are referred to the $\mathrm{C}$ band observations. The data are reported in Table 5.3. The best fit from the $\mathrm{X}$ data is $\mathrm{v}_{\mathrm{sep}}^{\mathrm{X}}=(0.28 \pm 0.02) \mathrm{c}$ (solid line), and the best fit from the $\mathrm{C}$ data is $\mathrm{v}_{\text {sep }}^{\mathrm{C}}=(0.26 \pm 0.02) \mathrm{c}$ (dots line). 


\section{$5.2 .2 \quad \mathrm{~J} 0111+3906$}

Multi-epoch observations of the MOJAVE project, measurements obtained by Owsianik et al., (1998) and the data used by Taylor et al., (2000), were taken in to account for the multi-epoch study of J0111+3906. All the data used for multi epoch analysis of the source J0111+3906 are presented in Table 5.4.

\begin{tabular}{|c|c|c|c|c|}
\hline \multicolumn{5}{|c|}{$\mathrm{J} 0111+3906$} \\
\hline \multicolumn{5}{|c|}{ Multi Epoch Observations } \\
\hline Epochs & $\underline{\mathrm{LS}}(\mu \mathrm{as})$ & $\underline{\sigma_{\mathrm{r}}(\mu \mathrm{as})}$ & Paper & $\underline{B a n d}$ \\
\hline 1982.9 & 5.69 & 0.1 & $\mathrm{~A}$ & $\mathrm{C}$ \\
\hline 1984.8 & 5.70 & 0.1 & $\mathrm{~A}$ & $\mathrm{C}$ \\
\hline 1986.7 & 5.72 & 0.1 & $\mathrm{~A}$ & $\mathrm{C}$ \\
\hline 1994.4 & 5.81 & 0.07 & $\mathrm{D}$ & $\mathrm{U}$ \\
\hline 1996.4 & 5.82 & 0.07 & $\mathrm{D}$ & $\mathrm{U}$ \\
\hline 1997.6 & 5.87 & 0.07 & $\mathrm{D}$ & $\mathrm{U}$ \\
\hline 1999.8 & 5.86 & 0.06 & $\mathrm{~B} / \mathrm{D}$ & $\mathrm{U}$ \\
\hline 2002.4 & 5.85 & 0.06 & $\mathrm{~B}$ & $\mathrm{U}$ \\
\hline 2006.3 & 5.78 & 0.06 & $\mathrm{~B}$ & $\mathrm{U}$ \\
\hline 2008.3 & 5.82 & 0.10 & $\mathrm{C}$ & $\mathrm{C}$ \\
\hline 2008.3 & 5.88 & 0.08 & $\mathrm{C}$ & $\mathrm{X}$ \\
\hline 2009.3 & 5.84 & 0.06 & B & $\mathrm{U}$ \\
\hline 2012.9 & 5.91 & 0.06 & B & $\mathrm{U}$ \\
\hline
\end{tabular}

Table 5.4: Data from the multi-epoch observations of J0111+3906. Column 1: epoch of the observations, Column 2: Linear size measured in $\mu$ as, Column 3: error $\sigma_{r}$ estimated according to Eq.4.3 in $\mu \mathrm{as}$, Column 4: references (A: Owsianik et al., 1998, B: Mojave dataset (Lister et al., 2003), C: this work, D: Taylor et al., (2000) ), Column 5: frequency band of the observations. 
The best fit plot of the data reported in Table 5.4 is shown in Fig. 5.3 considering separately the observing frequency ( $\mathrm{C}$ and $\mathrm{U}$ band).

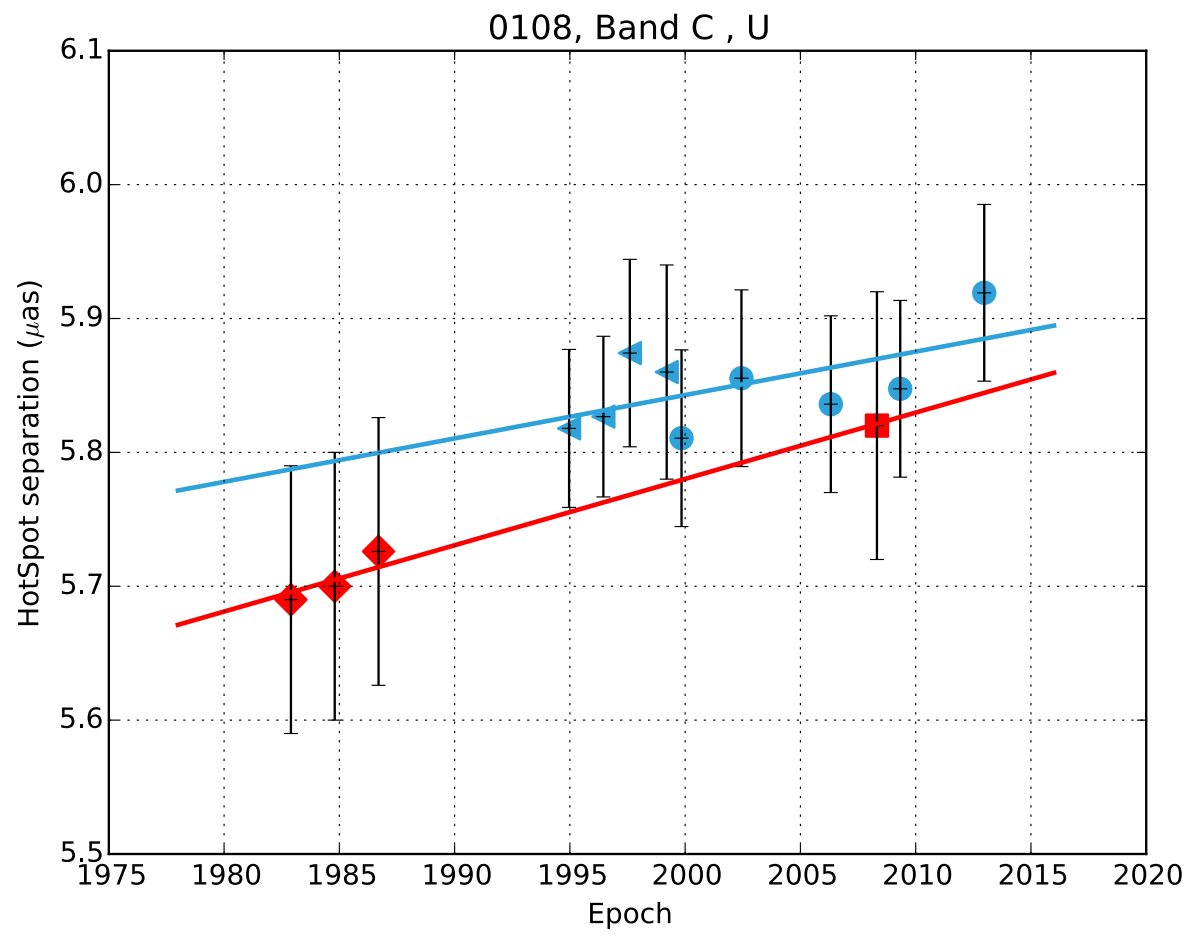

Figure 5.3: Plot of the hot spot separation versus the observing epoch for J0111+3906. Light blu symbols represent the data taken in $\mathrm{U}$ band, the red symbols are observation taken in $\mathrm{C}$ band. Square and circles are relative to the observations used in this work, the diamonds are relative to the work by Owsianik et al (1998) and the triangles represent the observations from the paper by Taylor et al., (2000). 
The light blue line in Fig. 5.3 represents the best fit at $15 \mathrm{GHz}$ and its slope is the hot spot separation speed $\mathrm{v}_{\mathrm{sep}}$. From the nine-epoch observations taken into consideration in U band I derived:

$$
\mathrm{v}_{\mathrm{sep}}^{\mathrm{U}}=(3.31 \pm 1.8) \mu \text { as } \mathrm{y}^{-1}
$$

which corresponds to

$$
\mathrm{v}_{\mathrm{sep}}^{\mathrm{U}}=(0.046 \pm 0.025) \mathrm{c}
$$

From the four epoch of observations in $\mathrm{C}$ band I derived:

$$
\mathrm{v}_{\text {sep }}^{\mathrm{C}}=(4.95 \pm 3.2) \mu \text { as } \mathrm{y}^{-1}
$$

which corresponds to

$$
\mathrm{v}_{\text {sep }}^{\mathrm{C}}=(0.06 \pm 0.04) \mathrm{c}
$$

The $5 \mathrm{GHz}$ observations were compared with the work by Owsianik et al., (1998). Owsianik et al., (1998), found that the hot spots in J0111+3906 have a separation velocity of about $\mathrm{v}_{\text {sep }}^{\mathrm{C}}=(0.25 \pm 0.04) \mathrm{c}$. The difference in this case are probably due to the different instrument used. In fact Owsianik et al., (1998) considered three epochs of Global VLBI observations at $5 \mathrm{GHz}$ spread over 12 years. The Global VLBI, has a better angular resolution with respect to the VLBA at $5 \mathrm{GHz}$, resulting in a more precise measure of the position of each hot spot in J0111+3906.

A further comparison can be made considering the work of Taylor et al., (2000) on J0111+3906. In this case the authors analyzed four VLBA epoch of observations taken between 1994 and 1999 at $15 \mathrm{GHz}$. The hot spot separation speed obtained by Taylor et al., (2000) was $\mathrm{v}_{\mathrm{sep}}^{\mathrm{U}}=(0.15 \pm 0.03) \mathrm{c}$ that is different with the one found in this work. This difference is relative to the number of epochs considered. In this work the hot spot separation speed was derived on nine epochs over eighteen years instead of the four epochs spanning five years considered in Taylor et al., (2000). The error on the hot spot separation speed presented in Fig. 5.3 is smaller than in Taylor et al., (2000) and this is due to the increased number of epochs taken into consideration in this work.

There is also a difference between the results obtained in $\mathrm{C}$ and $\mathrm{U}$ band observations. This may be due to the fact that in $\mathrm{U}$ band, the resolution is better, the source is optically thin and the results may be determined with less uncertainties.

We remark that a similar difference was found in the multi epoch analysis of J1944+5448, previously discussed (Section 5.2.1).

J0111+3906 expanded of about $\sim 60 \mu$ as from 1994 to 2012 which corresponds to 0.42 pc over eighteen years. The low expansion rate of J0111+3906 may indicate the presence of dense medium embedding the compact source. This aspect will be discussed in Section 
5.4.2 in more details.

\section{$5.3 \quad$ Age}

As discussed in Chapter 2, one of the main parameters we need to estimate in order to improve our knowledge about compact symmetric objects, is their age. I used the two methods described in Section 2.7 in order to obtain the age of the two CSO. The results are presented in the next Section.

\section{$5.3 .1 \quad \mathrm{~J} 1944+5448$}

I estimated the kinematic age of J1944+5448 by dividing the projected source linear size (LS) by the measured projected hot spot separation speed ( $\mathrm{v}_{\text {sep }}$ ) according to Eq. 2.20. For the source J1944+5448 I found:

$$
\mathrm{t}_{\text {kin }}=(1800 \pm 300) \mathrm{yr}
$$

This result is consistent with that found by Polatidis \& Conway (2003) who calculated a kinematic age of about $t_{\text {kin }} \sim 1400 \mathrm{yr}$ (once out cosmology is adopted). The differences may be related to the difference in the hot spot separation speed estimation (see Section 5.2.1), and in the frequency used as discussed in the previous Sections.

Then I estimated the radiative age of J1944+5448 according to Eq. 2.15.

As discussed in Chapter 2, the spectral age can be computed by using the break frequency of the lobes of the radio sources. The lobes are filled by electrons which are ageing, losing energy as the time passes. This produces a break in the radio spectrum in the optically thin range, which is strictly correlated with the age of the electron population. In particular synchrotron losses deplete high-energy electrons, leading to a steepening in the spectrum.

The spectrum of the source component is derived from multi frequency images. In order to derive the radiative age of J1944+5448 I used the results obtained by Murgia (2003) who fitted the spectrum of each component of J1944+5448. Murgia (2003) analyzed VLBA observations in L, C and X band (two separate frequencies for each band) in order to derive the local spectrum in various regions of the radio source. Murgia (2003) restricted the spectral age calculation to the west lobe of J1944+5448 which is brighter and permits to measure the break frequency closer to the core and therefore to test older electrons.

(Murgia, 2003) found that the spectra of the lobes are well fitted by a JP model (single injection of particles) while the hot spots are better fitted by a power law as predicted by theoretical studies.

The oldest electrons have a break at $\nu_{\mathrm{br}} \sim 4 \mathrm{GHz}$ and such value has been used to derive 
the radiative age. For its determination we assumed the lobe equipartition magnetic field (see Section 5.1.1).

The parameters used in estimating the spectral age of J1944+5448 are the following:

- Break frequency: $\nu_{\text {break }} \sim 4 \mathrm{GHz}$ (Murgia, 2003)

- Equipartition magnetic field: $\mathrm{H}_{\mathrm{eq}} \sim 2.7 \mathrm{mG}$

- Redshift: $\mathrm{z}=0.263$

thus the resulting spectral age is:

$$
\mathrm{t}_{\mathrm{rad}}=(1900 \pm 500) \mathrm{yr}
$$

The spectral age estimated by Murgia was $\mathrm{t}_{\mathrm{rad}}^{\mathrm{Murgia}} \sim 1500 \mathrm{yr}$ which is consistent with the one found in this work. The difference may be related to the different size of the lobe measured in the two cases. In fact the angular size of the lobe derived in this work (Table 4.2.1) is $1.5 \times 1.3 \mathrm{mas}^{2}$ while the one used in Murgia (2003) is $20 \times 10 \mathrm{mas}^{2}$. As previously discussed the magnetic field strictly depends on the volume. Therefore some difference in the estimation of the the volume results in a different magnetic field.

However, the comparison between the kinematic age

$$
\mathrm{t}_{\text {kin }} \sim 1800 \mathrm{yr}
$$

and the radiative age

$$
\mathrm{t}_{\mathrm{rad}} \sim 1900 \mathrm{yr}
$$

shows that these results are in good agreement.

In conclusion our data confirm that $\mathrm{J} 1944+5448$ is a young radio source with an age of about $\sim 10^{3} \mathrm{yr}$. 


\subsubsection{J0111+3906}

For the source J0111+3906 only the kinematic age was estimated, since in this source the lobes could not be imaged in sufficient detail for estimating the break frequency of the lobes. I derived the kinematic age according to Eq. 2.20, and I found :

$$
\mathrm{t}_{\mathrm{kin}}=(1080 \pm 400) \mathrm{yr}
$$

A comparison can be made considering the kinematic age derived in Taylor et al., (2000), who found $t_{\text {kin }}=(310 \pm 70) \mathrm{yr}$. It is worth noting that the different hot spot separation speed derived (Section 5.2.2) may produce different kinematic ages. Therefore a more precise estimate of the linear size LS and therefore of the hot spot advance speed, would result in a more precise estimate of the age of these sources. This is possible considering a large number of epoch of observation, which would reduce the error on the linear size, and thus the error on $\mathrm{v}_{\text {sep }}$ calculation. As J1944+5448 (Section 5.3.1) also $\mathrm{J} 0111+3906$ is a young radio source, with an age of about $\mathrm{t}_{\mathrm{kin}} \sim 10^{2-3} \mathrm{yr}$. It is worth mentioning that a further comparison with the spectral age would be useful for better to constraining the age of J0111+3906. We note that the kinematic age is a measure which depends on the instantaneous hot spot separation rate that, as discussed in Chaper 2, may be not truly representative of the mean growth rate of the source. 


\section{$5.4 \quad$ Symmetries}

Asymmetries observed in the morphology of compact objects may be due to inhomogeneities in the ambient medium embedding the radio source (Chapter 2). Here the analysis of the symmetries of the two CSO is presented.

\section{$5.4 .1 \quad \mathrm{~J} 1944+5448$}

We consider the core position as a reference and then the source expansion on the two opposite size can be determined, $\mathrm{v}_{\mathrm{W}-\mathrm{HS}}^{\mathrm{c}}$ and $\mathrm{v}_{\mathrm{E}-\mathrm{HS}}^{\mathrm{c}}$ for the West and East hot spots respectively according to Eq. 5.7:

$$
\mathrm{v}_{\mathrm{HS}}^{\mathrm{c}}=\frac{L S^{\mathrm{c}}}{\mathrm{t}_{\mathrm{kin}}}
$$

where LS $^{\mathrm{c}}$ is the distance between the core and the hot spots and $t_{\text {kin }}$ is the kinematic age estimate in Section 5.3.1. I obtained that the West hot spot has a velocity of

$$
\mathrm{v}_{\mathrm{W}-\mathrm{HS}}^{\mathrm{c}}=0.05 \mathrm{c}
$$

with respect to the core, while the East hot spot speed is

$$
\mathrm{v}_{\mathrm{W}-\mathrm{HS}}^{\mathrm{c}}=0.19 \mathrm{c}
$$

I estimated the external density of the ambient medium assuming an equilibrium between the equipartition pressure $\mathrm{P}_{\mathrm{eq}}$ (Eq. 5.6) and the pressure of the ISM. The pressure of the ISM may be separated in two component:

the static pressure

$$
\mathrm{P}_{\mathrm{ISM}}=2(\Gamma-1) \frac{3}{2} \mathrm{nkT}
$$

where $\mathrm{n}$ is the density, $\mathrm{T}$ is the temperature, $\mathrm{k}$ is the Boltzman constant and $\Gamma$ is the heat capacity ratio $(5 / 3)$,

and the dynamic pressure:

$$
\mathrm{P}_{\mathrm{ram}}=\mathrm{m}_{\mathrm{p}} \mathrm{n}_{\text {ext }}\left(\mathrm{v}_{\mathrm{HS}}^{\mathrm{c}}\right)^{2}
$$

where $\mathrm{m}_{\mathrm{p}}$ is the proton mass $\left(\mathrm{m}_{\mathrm{p}}=1.2710^{-24} \mathrm{gr}\right), \mathrm{v}_{\mathrm{HS}}^{\mathrm{c}}$ is the hot spot separation speed with respect to the core and $\mathrm{n}_{\text {ext }}$ is the external density in part $/ \mathrm{cm}^{3}$.

In the case of the ISM of J1944+5448 the temperature is found to be $\sim 10^{4} \mathrm{~K}$, therefore the term $\mathrm{P}_{\text {ISM }}$ should be negligible with respect to the equilibrium pressure if usual values for the density of the external medium are assumed (Marr et al., 1998). In particular in this case $\mathrm{P}_{\mathrm{ISM}} \sim 1.310^{-9}$ dyne $/ \mathrm{cm}^{2}$ indeed I adopted $\mathrm{P}_{\mathrm{eq}}=\mathrm{P}_{\mathrm{ram}}$. 
Then, with these values I calculated the external medium density, which is:

$$
\mathrm{n}_{\text {ext }}^{\mathrm{W}}=48.4 \mathrm{part} / \mathrm{cm}^{3}
$$

in the West region and

$$
\mathrm{v}_{\text {ext }}^{\mathrm{E}}=3.5 \text { part } / \mathrm{cm}^{3}
$$

for the East one.

Therefore, the density of the ambient medium in the West part is more than an order of magnitude higher than in the East region. This agreed with the lower separation speed estimate for the West hot spot. The high density medium on the West region may have slowed down the hot spot propagation in that direction. On the contrary the East hot spot, interacting with a lower density medium is faster advancing through it. This may have created the asymmetric shape of the source J1944+5448. According to time delay arguments from an intrinsically symmetric source orientated at a given angle to the l.o.s. (Orienti et al. 2007), the brightest hot spot should be the farthest from the core. On the contrary, the brightest hot spot in J1944+5448 is the closer to the core with a slower advance speed, and geometrical effects do not reproduce this kind of asymmetry. In conclusion, the asymmetric shape of J1944+5448 is due to inhomogeneities in the ambient medium in which the source is completely embedded (Wagner et al., 2012). 


\section{$5.4 .2 \quad \mathrm{~J} 0111+3906$}

The source J0111+3906, as discussed in Section 5.3.2, is asymmetric and the North East region is the closer one to the core. Considering the high resolution images (Fig. 4.9), I derive the hot spot separation speed with respect to the core according to Eq.5.7. I obtained that the South West hot spot has

$$
\mathrm{v}_{\mathrm{SW}-\mathrm{HS}}^{\mathrm{c}}=0.05 \mathrm{c}
$$

and that the North East hot spot has

$$
\mathrm{v}_{\mathrm{NE}-\mathrm{HS}}^{\mathrm{c}}=0.01 \mathrm{c}
$$

In this case the hot spot closer to the core, the NE, is also the one with the slower speed, as in the case of the source J1944+5448. According to Marr et al., (2000), $\mathrm{P}_{\text {ISM }} \sim 10^{-15}$ dyne $/ \mathrm{cm}^{2}$ assuming $\mathrm{n} \sim 10^{3} \mathrm{~cm}^{3}$ and $\mathrm{T} \sim 10^{4} \mathrm{k}$ and is negligible (Marr et al., 2001). Therefore, considering an equilibrium condition between the ram pressure and the equipartition pressure of the source component I derived the external density of the ambient medium. I found that the gas external density is

$$
\mathrm{n}_{\mathrm{ext}}^{\mathrm{SW}}=2.09 \text { part } / \mathrm{cm}^{3}
$$

in the South West region, while it is

$$
\mathrm{n}_{\mathrm{ext}}^{\mathrm{NE}}=23.3 \text { part } / \mathrm{cm}^{3}
$$

in the East region. This means that the ambient medium is denser in the North East region and has slow down the hot spot advance in that direction. This produced the asymmetric shape of J0111+3906. It is worth noting that this is a compact object, with a linear size of about $40 \mathrm{pc}$ and that is completely embedded in the innermost region of the host galaxy. Furthermore we can compare this result with the one found by Owsianik et al., (1998) according to which the upper limit on the external density is about $2.1 \mathrm{~cm}^{-3}$ and that is in agreement with what found for the SW region (Owsianik et al., 1998). 


\subsection{Spectral Analysis}

\subsubsection{J1944+5448}

Since only two frequencies were available for the source J1944+5448, it was not possible to derive the radio spectrum profile of each source region. However, it is possible to derive some information from the spectral index image. In $\mathrm{C}$ and $\mathrm{X}$ band the source is optically thin. The flux density decreases from the $\mathrm{C}$ to the $\mathrm{X}$ band. The literature information confirm that J1944+5448 has a turnover at low frequency, around $1 \mathrm{GHz}$ (Snellen et al., 1998). The total radio spectrum of this source is shown in Fig. 5.4 According to the spectral classification described in Section 2.3, J1944+5448 may be defined as a GPS.

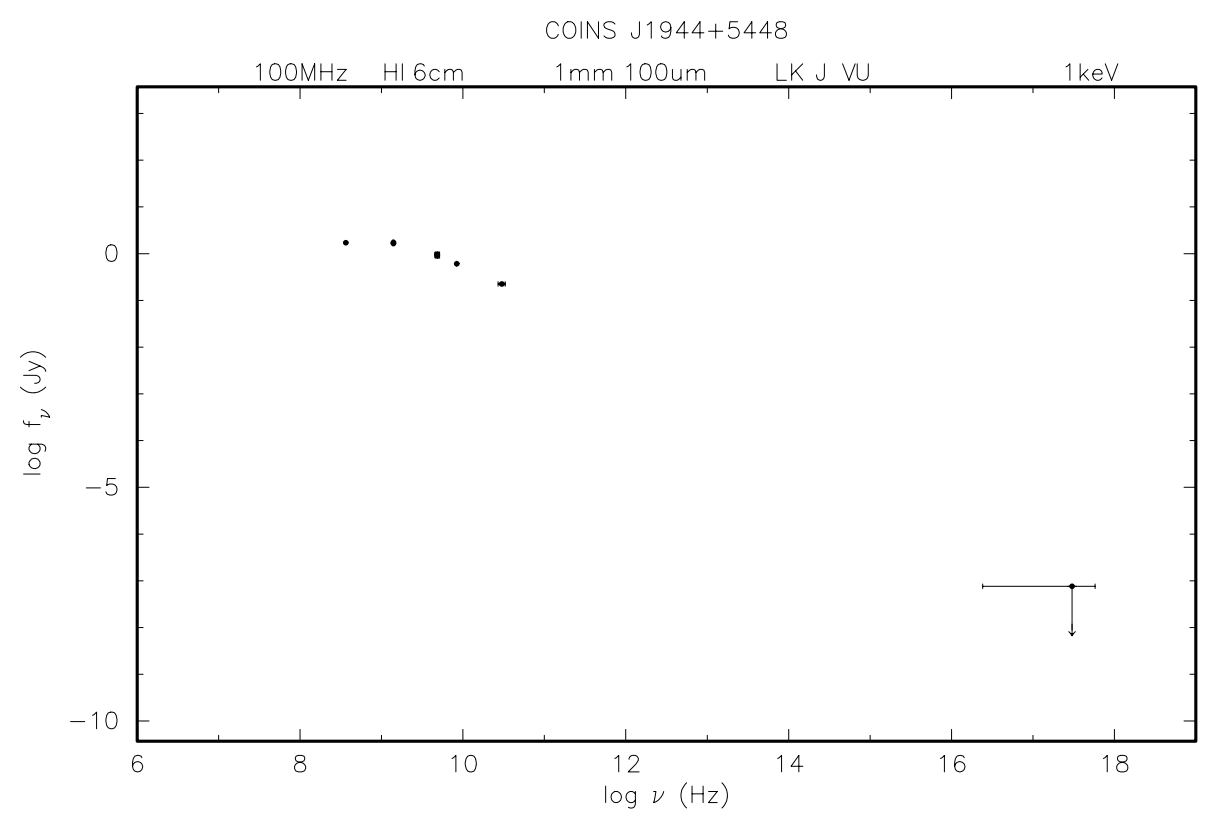

Figure 5.4: Radio spectrum of the source J1944+5448. Values are from the Nasa extragalactic database (NED). 


\section{$5.5 .2 \quad \mathrm{~J} 0111+3906$}

I focus on the spectral analysis of the source J0111+3906 since there are 4 frequency bands available from which we can derive the radio spectrum. The flux density of each component in L, C, X and $\mathrm{U}$ band is reported in Table 5.5 and Table 5.6.

\begin{tabular}{lll}
\hline \multicolumn{3}{c}{ North-East region } \\
\hline$\nu(\mathrm{GHz})$ & $\mathrm{S}(\mathrm{mJy})$ & $\sigma_{\mathrm{S}}$ \\
\hline 1.411 & 207 & 22.3 \\
1.639 & 248.4 & 19.9 \\
4.546 & 891.2 & 71.2 \\
4.88 & 828.7 & 66.5 \\
8.11 & 674.2 & 58.1 \\
8.48 & 654.1 & 52.3 \\
15.33 & 228.1 & 12 \\
\hline
\end{tabular}

Table 5.5: Data of the North East region of J0111+3906. Column 1: frequency in GHz, Column 2: flux density in mJy, Column 3: error on the flux density in mJy calculated according to Eq.4.2.

\begin{tabular}{lll}
\hline \multicolumn{3}{c}{ South -West region } \\
\hline$\nu(\mathrm{GHz})$ & $\mathrm{S}(\mathrm{mJy})$ & $\sigma_{\mathrm{S}}$ \\
\hline 1.411 & 239.3 & 23.1 \\
1.639 & 260.1 & 26 \\
4.546 & 513.2 & 54.9 \\
4.88 & 529.1 & 53.1 \\
8.11 & 354 & 35.1 \\
8.48 & 368.5 & 36 \\
15.33 & 199.1 & 12 \\
\hline
\end{tabular}

Table 5.6: Data of the South West region of J0111+3906. Column 1: frequency in GHz, Column 2: flux density in mJy, Column 3: error on the flux density in mJy calculated according to Eq.4.2. 
It is known that the convex radio spectrum of compact radio sources is probably due to the Synchrotron Self Absorption (SSA) as discussed in Chapter 2. For this reason, as a first step, I fit the spectra of the two main region of the source J0111+3906 with an SSA model.

\section{Synchrotron-Self Absorption Model}

I used the SSA model, described in Snellen et al., (1998).

The model function is:

$$
\mathrm{S}(\nu)=\left(\frac{\mathrm{S}_{\text {peak }}}{1-\mathrm{e}^{-1}}\right) \times\left(\frac{\nu}{\nu_{\text {peak }}}\right)^{\alpha_{\text {thcik }}} \times\left(1-\mathrm{e}^{-\left(\frac{\nu}{\nu_{\max }}\right)^{\left(\alpha_{\text {thin }}-\alpha_{\text {thick }}\right)}}\right)
$$

In Eq.5.8:

- $\left.\mathrm{S}_{(} \nu\right)$ is the flux density,

- $\mathrm{S}_{\text {peak }}$ is the maximum flux density,

- $\nu_{\text {peak }}$ is the frequency that corresponds to the maximum flux density,

- $\alpha_{\text {thick }}$ is the optically thick spectral index,

- $\alpha_{\text {thin }}$ is the optically thin spectral index.

Eq.5.8 represents a homogeneous synchrotron self absorbed radio source for $\alpha_{\text {thick }}=2.5$ (Moffett et al., 1995). In this model $\alpha_{\text {thin }}, \alpha_{\text {thick }}, \nu_{\text {peak }}$ and $\mathrm{S}_{\text {peak }}$ are free parameters (Snellen et al., 1998).

The results about the SSA model-fit of the spectra of the NE and SW regions in J0111+3906 are shown in Fig. 5.5 and Fig. 5.6. 


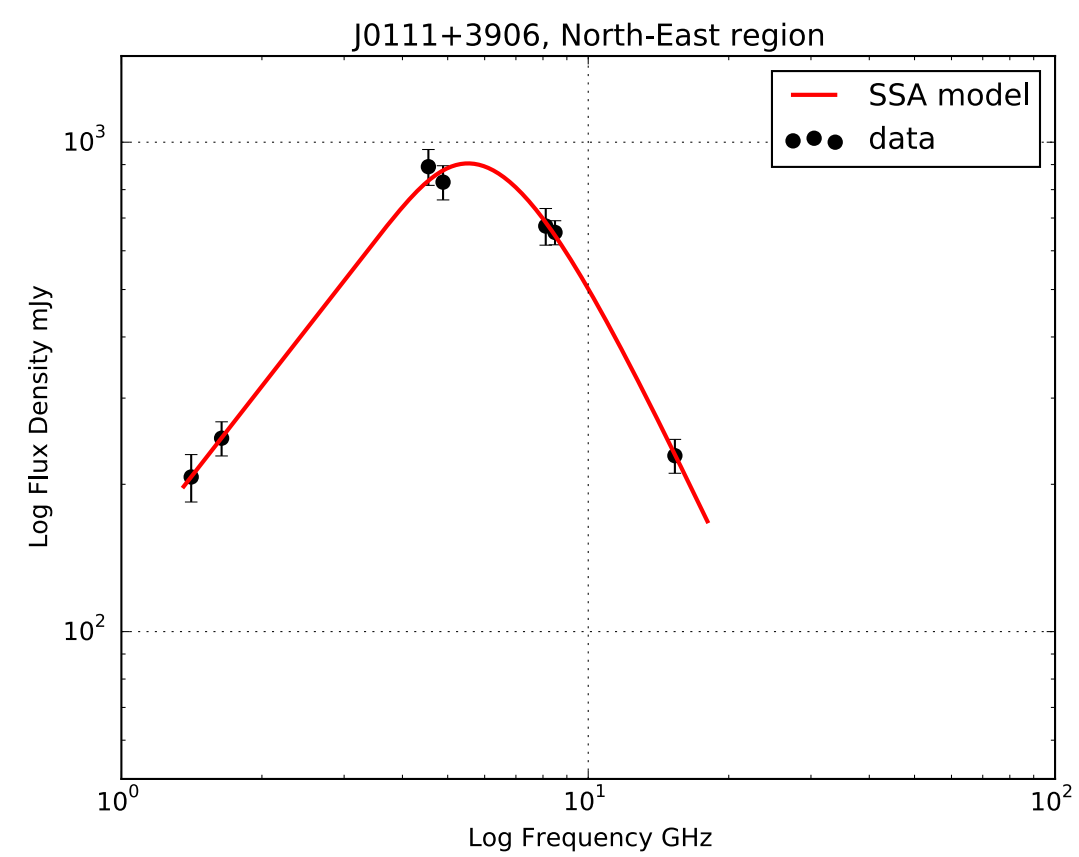

Figure 5.5: Radio spectrum of the NE region of J0111+3906 fitted with the SSA model according to Eq. 5.8.

In this case of the NE component, the parameters of the fit are:

- $\mathrm{S}_{\text {peak }}=(854.3 \pm 24.03) \mathrm{mJy}$

- $\nu_{\text {peak }}=(6.5 \pm 0.9) \mathrm{GHz}$

- $\alpha_{\text {thick }}=1.22 \pm 0.12$

- $\alpha_{\text {thin }}=-2.03 \pm 1.15$

the $\chi^{2}$ of the fit is $\chi^{2}=1.18$ while the reduced chi square is $\chi_{R}^{2}=0.39$.

In this case we notice that the North-East component has a convex radio spectrum with a turnover at $6.5 \mathrm{GHz}$. In the optically thick region, the NE component is selfabsorbed with a spectral index $\alpha_{\text {thick }} \sim 1.22$. On the other hand, in the optically thin region the spectrum is steeper with a spectral index $\alpha_{\text {thin }} \sim-2.03$. The latter is different from the values obtained from the spectral index image between $\mathrm{C}$ and $\mathrm{X}$ band (Section 4.1.2). Since the turnover of the spectrum of the NE region occurs between 4.8 and 8.4 $\mathrm{GHz}$, the spectral index images in this frequency range is not a good estimator of the optically thin region of the spectrum. On the other hand, in the case of the optically thick 
spectral index, as discussed in Section 4.3.2, from the images we found $0.9<\alpha_{\text {thick }}<1.19$ which is comparable with the one found with the SSA model.

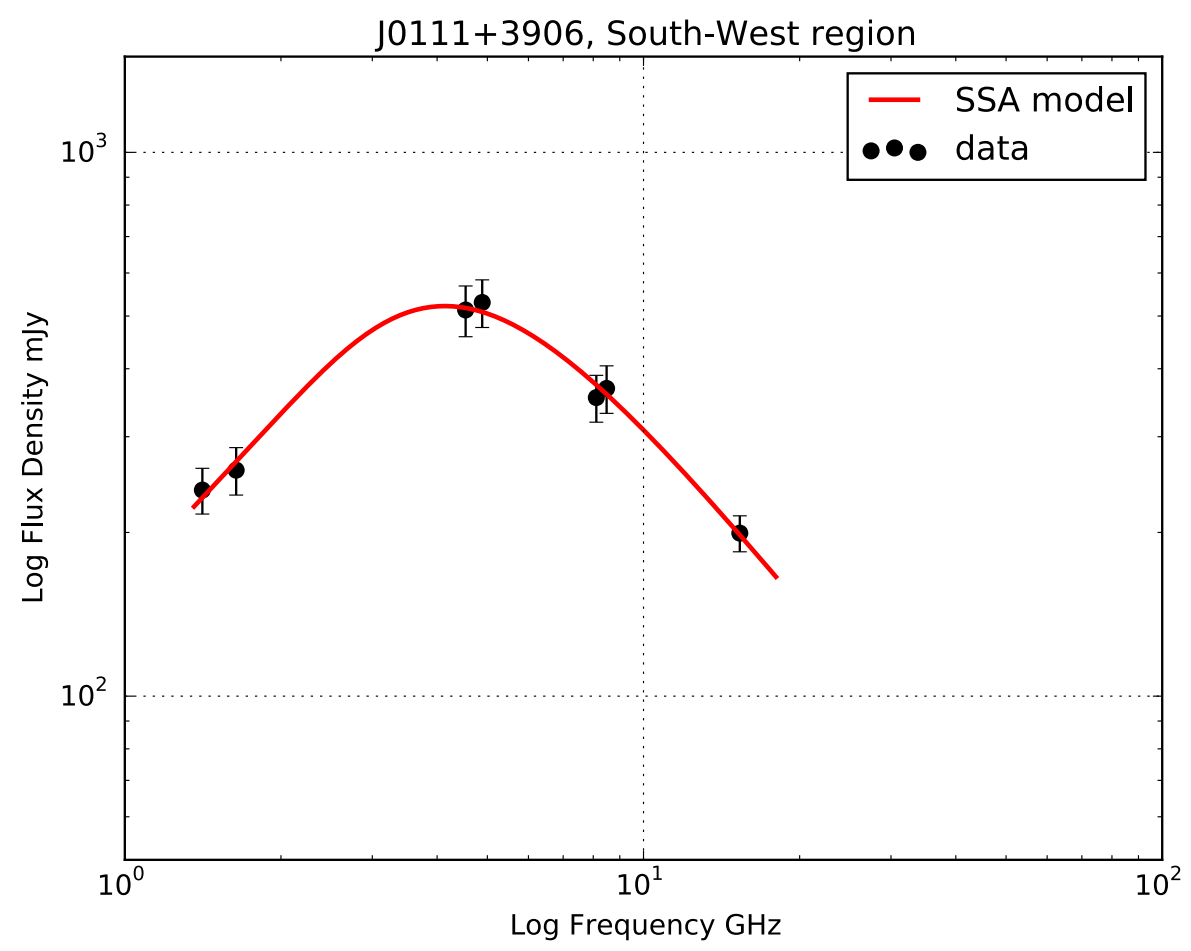

Figure 5.6: Radio spectrum of the SW region of J0111+3906 fitted with the SSA model according to Eq. 5.8.

In the case of the SW component, the parameters of the fit are:

- $\mathrm{S}_{\text {peak }}=(511.8 \pm 24.6) \mathrm{mJy}$

- $\nu_{\text {peak }}=(4.8 \pm 0.74) \mathrm{GHz}$

- $\alpha_{\text {thick }}=1.02 \pm 0.12$

- $\alpha_{\text {thin }}=-1.28 \pm 0.25$

the $\chi^{2}$ of the fit is $\chi^{2}=0.79$ while the reduced chi square is $\chi_{R}^{2}=0.26$.

In the case of the South West component, the turnover occurs at $4.8 \mathrm{GHz}$ with a peak flux density of $\sim 500 \mathrm{mJy}$. This component is weaker than the NE region. In addition in this case the optically thin spectral index is in agreement with the one found with the 
spectral indices images (Fig.4.12) in which we found $\alpha_{\text {thin }} \sim-1.2$. The optically thick spectral index $\alpha_{\text {thick }}$ agrees with the spectral indice images that provide $\alpha_{\text {thick }} \sim 1.1$.

The differences between the $\alpha_{\text {thick }}$ and $\alpha_{\text {thin }}$ values obtained by the fit and those found by the spectral index images are related to the fact that the spectral index image made use of only two points for each image (the pair frequency considered) while this fit uses seven points. Therefore the results may be not match perfectly the spectral indices images result.

The turnover difference between the North East and the South West region may be due to the presence of an extended lobe-like region in the SW component. The presence of electrons that are ageing in the lobe may shift the turnover to lower frequency, as observed in the SW component.

The measured spectral index between $\mathrm{L}$ and $\mathrm{C}$ bands confirms the results obtained by these fits. Between the $\mathrm{L}$ and $\mathrm{C}$ band the source radio spectrum is self-absorbed, while between the $\mathrm{C}, \mathrm{X}$ and $\mathrm{U}$ band it is optically thin and the spectral index ranges between $\alpha_{\text {thin }} \sim-1.1$ and $\sim-0.8$ froms the SW to NE region. The source spectrum of J0111+3906 has turns over around $\sim 5 \mathrm{GHz}$ and thus, according to the spectral classification described in Section 2.3 it may be classified as a High Frequency Peakers (HFP) radio source.

Once we obtained the SSA parameters, thus the turnover frequency and the peak flux density, we have an independent way to derive the magnetic field by using the SSA model:

$$
\mathrm{H}_{\mathrm{SSA}}=\mathrm{f}^{-5} \theta_{\text {maj }}^{2} \theta_{\text {min }}^{2} \nu_{\text {to }}^{5} \mathrm{~S}_{\text {peak }}^{-2}(1+\mathrm{z})^{-1}
$$

where $\theta_{\text {maj }}$ and $\theta_{\min }$ are the major and minor axis respectively of the source component, $\nu_{\text {to }}$ is the turnover frequency, $(1+\mathrm{z})^{-1}$ is the k-correction, $\mathrm{S}_{\text {peak }}$ is the peak flux density and $\mathrm{f}$ is a function which slightly depends on the frequency. In this work, I assumed $\mathrm{f} \approx 2$. It is worth mentioning that the magnetic field estimated in this way depends on high power of the parameters and therefore small error on their estimations may produce a large uncertainty in the magnetic field. The reliability of the SSA magnetic field can be set to the order of magnitude and with this condition we can made the comparison with the magnetic field estimated with the equipartition. 
By means of Eq. 5.9 I derived the magnetic field in each source component, considering the peak flux density previously obtained from the SSA fit.

$$
\begin{aligned}
& \mathrm{H}_{\mathrm{SSA}}^{\mathrm{NE}} \sim 50 \mathrm{mG} \\
& \mathrm{H}_{\mathrm{SSA}}^{\mathrm{SW}} \sim 34 \mathrm{mG}
\end{aligned}
$$

These results well agree with the equipartition magnetic field presented in Section 5.1.2, that are

$$
\mathrm{H}_{\mathrm{eq}}^{\mathrm{NE}} \sim 39 \mathrm{mG}
$$

and

$$
\mathrm{H}_{\mathrm{SSA}}^{\mathrm{SW}} \sim 41 \mathrm{mG}
$$

This comparison may be useful in understanding if the SSA is the main mechanism responsible for the observed spectral turnover of the radio spectrum in this source.

Another mechanism that may be adopted in order to explain the spectrum of J0111+3906, is the Free Free absorption model (FFA). As discussed in Chapter 2, this mechanism could explain the convex radio spectrum of compact sources, assuming the presence of an ionized gas in the ambient medium in which these sources are completely embedded.

\section{Free Free Absorption Model}

I used the FFA model described in Marr et al., (2001) in order to fit the spectra of J0111+3906 components.

The optical depth of free free absorption depends on frequency according to:

$$
\tau_{\nu}=0.08253 \nu^{-2.1} \int \mathrm{T}_{\mathrm{e}}^{-1.35} \mathrm{n}_{\mathrm{e}}^{2} \mathrm{dl}
$$

where $\nu$ is the frequency in $\mathrm{GHz}, \mathrm{n}_{\mathrm{e}}$ is the electron density in unity of $\mathrm{cm}^{-3}, \mathrm{~T}_{\mathrm{e}}$ is the electron temperature $(\mathrm{k})$ and $\mathrm{dl}$ is the distance $(\mathrm{pc})$. The quantity $0.08253 \int \mathrm{T}_{\mathrm{e}}^{-1.35} \mathrm{n}_{\mathrm{e}}^{2} \mathrm{dl}$ is roughly the emission measure $\int \mathrm{n}_{\mathrm{e}}^{2} \mathrm{dl}$ times $\mathrm{T}_{\mathrm{e}}^{-1.35}$ times a constant. Hereafter this quantity will be termed as EMT ${ }^{-1.35}$. According to Eq. 5.10, the model adopted for FFA is the following:

$$
\mathrm{S}_{\nu}=\mathrm{S}_{0}\left(\frac{\nu}{1.53 \mathrm{GHz}}\right)^{\alpha} \exp \left(-\mathrm{EMT}^{-1.35} / \nu^{2.1}\right)
$$

where $\nu$ is the frequency, $\mathrm{S}_{\nu}$ is the flux density, $\mathrm{S}_{0}$ is the normalization of the flux density, $\mathrm{EMT}^{-1.35}$ is the quantity proportional to the emission measure as mentioned 
before.

In this model there are three free parameters, that are: $\mathrm{S}_{0}, \alpha$ and $\mathrm{EMT}^{-1.35}$. The plot of the FFA fit to the data is reported in Fig. 5.7 and Fig. 5.8, for the North East and South West regions, respectively. Finally, the comparison between the SSA and FFA model fit on the two regions of J0111+3906 is shown in Fig. 5.9 and Fig. 5.10, for the $\mathrm{NE}$ and SW components, respectively.

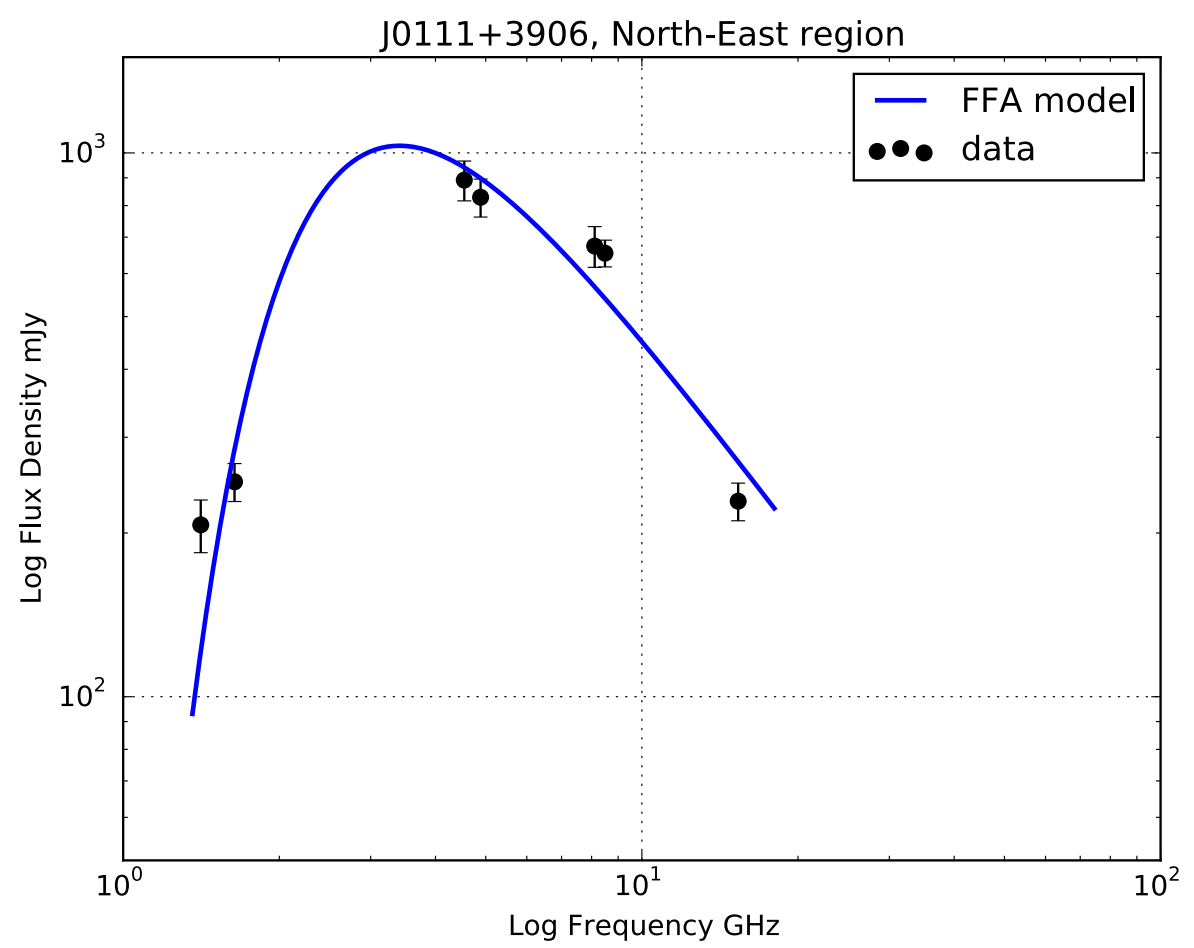

Figure 5.7: Radio spectrum of the NE region of J0111+3906 fitted with the FFA model according to Eq. 5.11.

In the case of the NE component, the results of the fit are:

- $\mathrm{S}_{\text {peak }}=1030.8 \mathrm{mJy}$

- $\nu_{\text {peak }}=3.40 \mathrm{GHz}$

- $\chi^{2}=36.9$

- $\chi_{R}^{2}=9.2$

According to the FFA model the North East region has a turnover at lower frequency, $\sim 3 \mathrm{GHz}$, than that obtained by the SSA fit and the peak flux density is about $\sim 1 \mathrm{Jy}$ instead of $\sim 850 \mathrm{mJy}$ for the SSA model. 


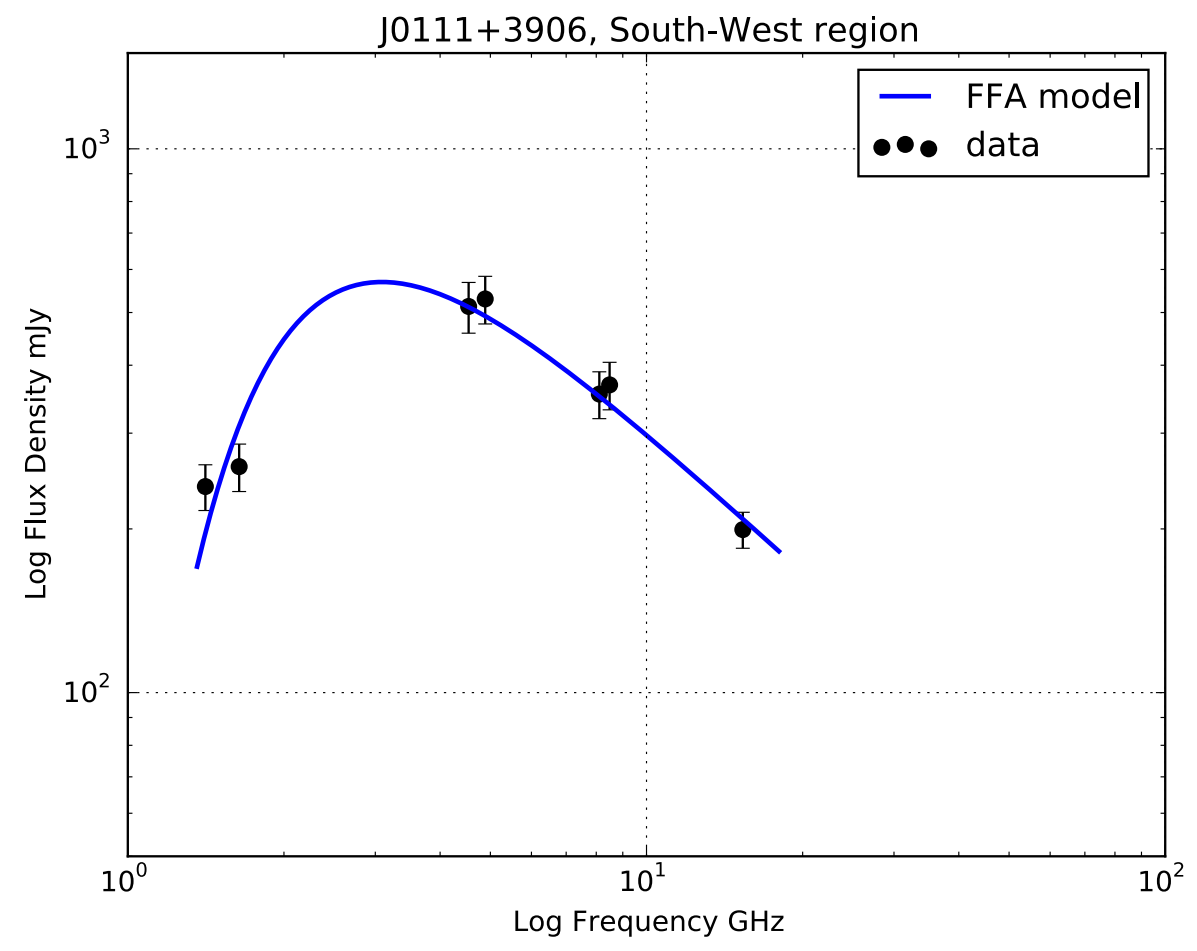

Figure 5.8: Radio spectrum of the SW region of J0111+3906 fitted with the FFA model according to Eq. 5.11.

In the case of the SW region, the results of the fit are:

- $\mathrm{S}_{\text {peak }}=568.9 \mathrm{mJy}$

- $\nu_{\text {peak }}=3.09 \mathrm{GHz}$

- $\chi^{2}=8.5$

- $\chi_{R}^{2}=2.12$

The South West region has a lower peak flux density $\sim 560$ mJy and a lower turnover frequency $(\sim 3 \mathrm{GHz})$ than that obtained by the SSA fit.

The comparison between the results of both the SSA and FFA model is provided in Fig. 5.9 and Fig. 5.10 for the NE ans SW region respectively. 


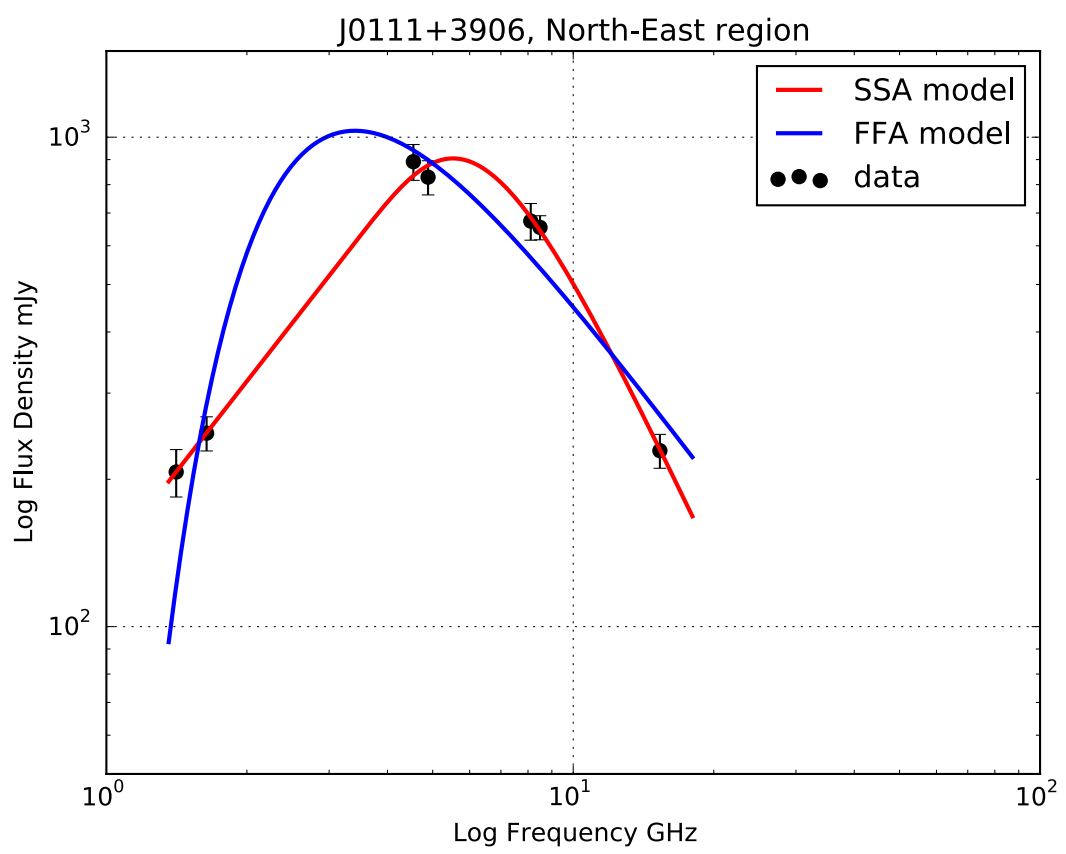

Figure 5.9: Radio spectrum of the NE region of the source J0111+3906. The blue line refers to the FFA model fit and the red line is the SSA model.

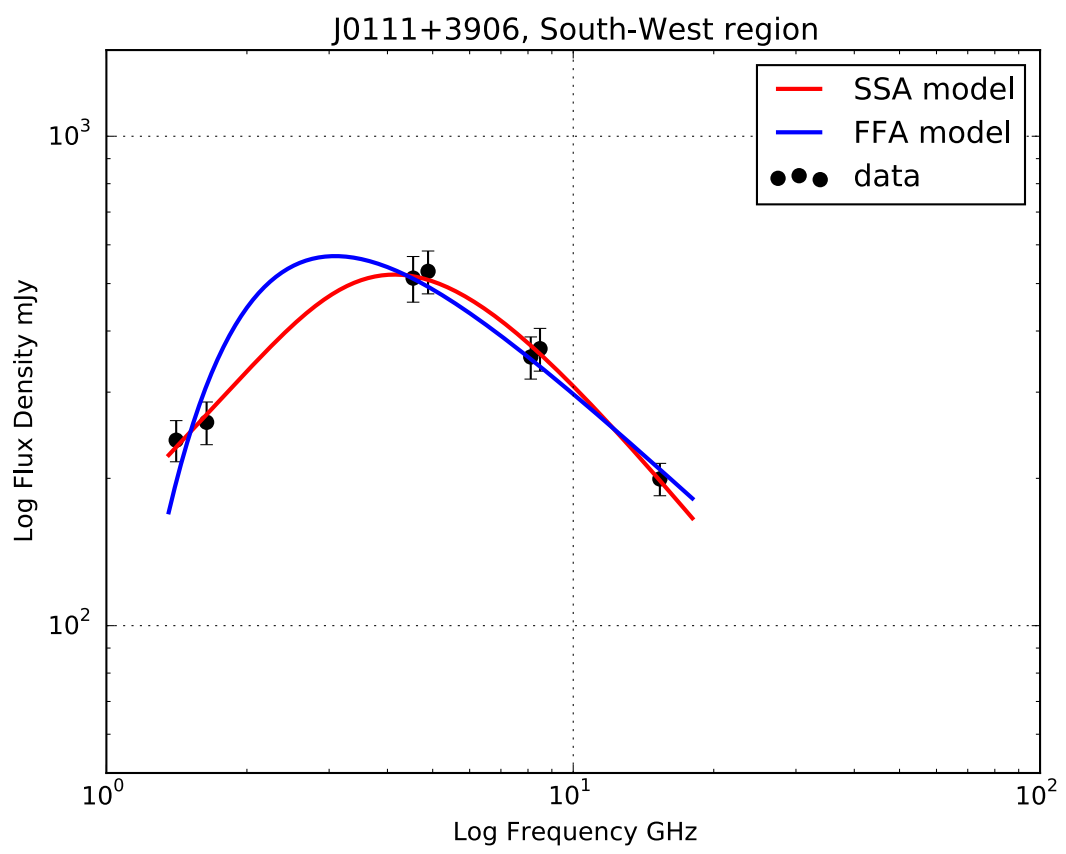

Figure 5.10: Radio spectrum of the SW component of J0111+3906. The red line refers to the SSA model fit and the blue line is the FFA model. 
According to Fig. 5.9 and Fig. 5.10, the radio spectra of the two regions of the source J0111+3906 are well fitted by an SSA model. In particular the two models have a completely different profile in the optically thick region. In the case of the optically thin part there is a smooth agreement for the SW component, while for the NE region the optically thin slopes fro SSA and FFA are different. In the case of FFA the turnover occurs at lower frequencies than that obtained with the $\mathrm{SSA}(\nu \sim 3 \mathrm{GHz})$.

In addition, in order to improve our knowledge of the source J0111+3906, I derived the optical depth $(\tau)$ images. These images, were obtained overlapping two images at different frequencies and using the task COMB. The task calculates the optical depth $\tau$ applying the following relation to each point of the radio image:

$$
\tau=\ln \left(\frac{S_{o b s}}{S_{e x p}}\right)
$$

where $S_{\text {obs }}$ is the observed flux density and $S_{\exp }$ is the expected flux density at a fixed value.

The images of the opacity distribution are provided in Fig. 5.11, Fig. 5.12 and Fig. 5.13 for L-C, C-X and X-U bands respectively. 


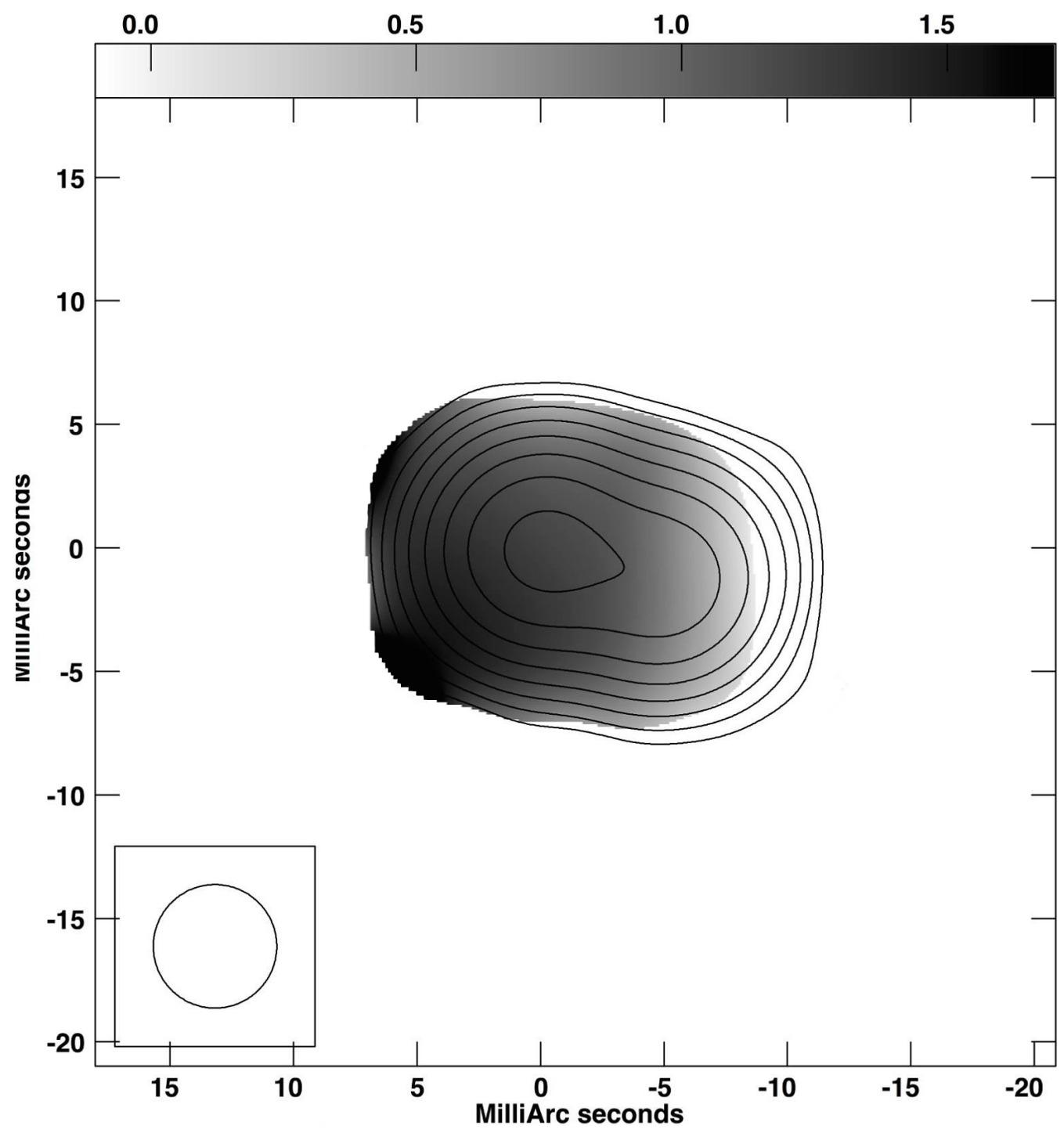

Figure 5.11: Optical depth distribution in J0111+3906 between 1.4 and $4.8 \mathrm{GHz}$. The contour level is the high frequency image ( $\mathrm{C}$ band) and the grey scale becomes darker toward larger optical depth $\tau$. 


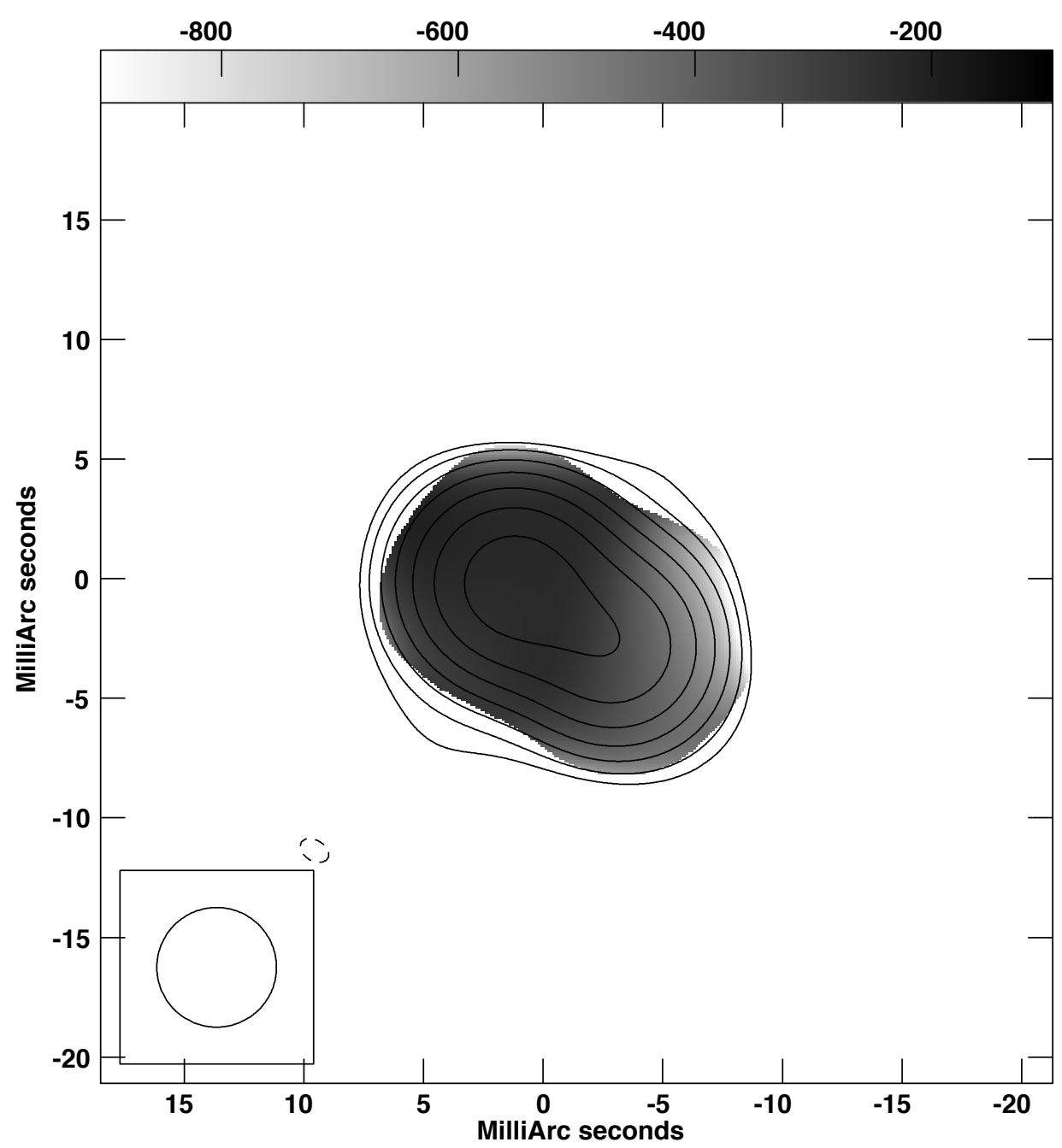

Figure 5.12: Optical depth distribution in J0111+3906 between 4.8 and 8.4 GHz. The contour level is the high frequency image (X band) and the grey scale becomes darker toward larger optical depth $\tau$. 


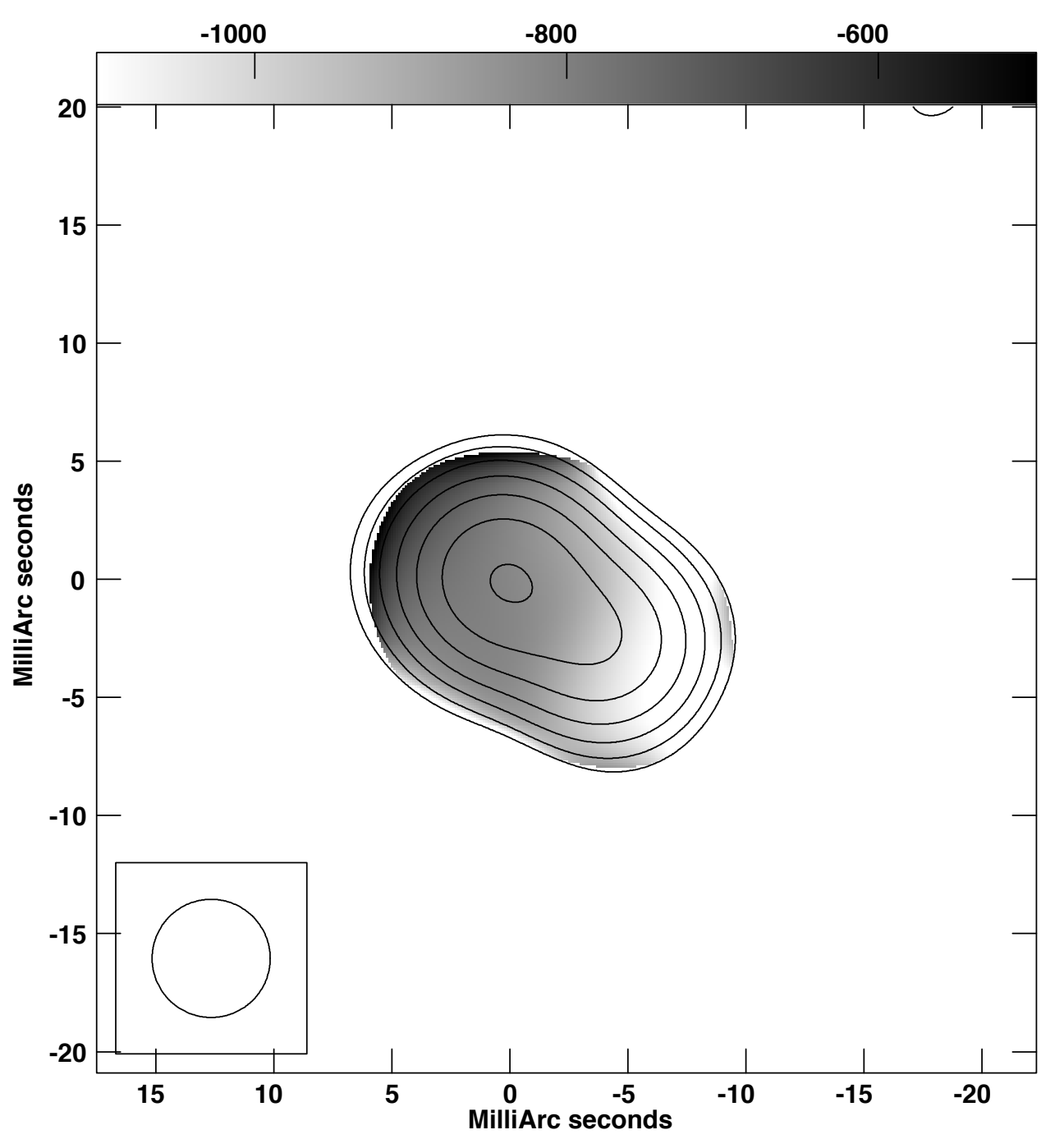

Figure 5.13: Optical depth distribution in J0111+3906 between 8.4 and $15 \mathrm{GHz}$. The contour level is the high frequency image ( $\mathrm{U}$ band) and the grey scale becomes darker toward larger optical depth $\tau$. 
Fig.5.11 provides the optical depth distribution between 1.4 and $4.8 \mathrm{GHz}$. In this frequency range, the NE region has $1.08<\tau<1.18$ while the SW has $0.9<\tau<1.01$. Between 4.8 and $8.4 \mathrm{GHz}$ the optical depth ranges between $-0.9<\tau<-0.2$ (Fig. 5.12). In particular the North East region has a lower opacity with $-0.5<\tau<-0.2$ while the South West part has $-0.9<\tau<-0.4$. Therefore between $\mathrm{C}$ and $\mathrm{X}$ band the $\mathrm{SW}$ region has a high opacity.

In the case of the analysis of the optical depth between 8.4 and $15.3 \mathrm{GHz}$ (Fig. 5.13), the opacity is lower than in the frequency range $4.8-8.4 \mathrm{GHz}$ as we expect since this is the optically thin region. In particular the North East region has an optical depth $-0.8<\tau<-0.5$ while the South West has $-1.2<\tau<-0.8$. Therefore each opacity image shows that the maximum absorption occurs at two point North and South of the center and that $\tau$ decreases along the direction perpendicular to the West-East direction. There is a decreasing optical depth from the low to the high frequencies.

Therefore considering the comparison between the synchrotron self absorption model and the free fee absorption model we can conclude that:

- The magnetic field assuming that the peak is due to the SSA $\left(\mathrm{H}_{\mathrm{SSA}}\right)$ for both the $\mathrm{NE}$ and SW region in J0111+3906 is in good agreement with the one found from equipartition conditions ( $\mathrm{H}_{\mathrm{Eq}}$.).

- Both the $\chi^{2}$ and the reduced $\chi_{R}^{2}$ are quite high in the FFA model fit, for each region of J0111+3906, providing a worse fit than SSA.

- The opacity maps between the L-X, C-X and X-U band, do not reveal any distribution of the optical depth across the source J0111+3906, which could suggest that the FFA may be the main mechanism which causes the spectrum turnover.

- The spectral indices $\left(\alpha_{\text {thick }}\right.$ and $\left.\alpha_{\text {thin }}\right)$ obtained with the SSA model are in good agreement with the spectral index images presented in Section 4.1.2.

From these results we favour the SSA as the main mechanism responsible for the observed spectral turnover in J0111+3906.

These results agree with what suggested by Artyukh et al., (2008) (Artyukh et al., 2008). 
On the contrary Marr et al., (2001) favoured the FFA model, for the following reasons:

- They observed that the whole source, or large part of it, in the case of FFA get fainter with decreasing frequencies as the optical depth of the foreground screen increases.

- They found that the peak spectral index in the optically thick part is $\alpha=3.2$ which is too high to be consistent with the SSA for which the theoretical model implies a spectral index of $\alpha=2.5$ in the rising part of the spectrum.

- SSA may cause the turnover in case of exceptionally large magnetic field (Philips \& Mutel 1984) that Marr et al., (2000) did not detect in J0111+3906.

Marr et al., (2000) made the hypothesis that a circumnuclear dense disk of ionized gas surrounding the source could produce free free absorption. This disk should have a temperature of about $10^{4} \mathrm{~K}$ and an electron density of about $\mathrm{n}_{\mathrm{e}}=10^{3} \mathrm{~cm}^{-3}$ extended over $100 \mathrm{pc}$ around the source.

In conclusion, we cannot exclude any mechanism on the basis of the spectral analysis and a combination of SSA and FFA may take place in the case of J0111+3906. In particular as also mentioned in Taylor et al., (2000) SSA could be useful in explaining the core inverted spectrum, while FFA may be involved in larger structures. In conclusion, our results favor the idea according to which the SSA model is the main mechanism at work and responsible for the observed turnover in J0111+3906. 


\section{$5.6 \mathrm{~J} 0111+3906$, really a young radio source?}

In Section 5.3.2 I estimated the age of J0111+3906, which turned out to be a young radio source $\left(10^{2-3} \mathrm{yr}\right)$. Baum et al., (1990), made observations of J0111+3906 at $49 \mathrm{~cm}$ and $6 \mathrm{~cm}$ with the Westerbork Synthesis Radio Telescope (WSRT) and at $18 \mathrm{~cm}$ with the VLA in order to analyze the radio diffuse emission from this source. They found a diffuse emission around J0111+3906 extending over the $\sim 120 \mathrm{kpc}$ scale, with a high spectral index $\alpha>2.4$ suggesting the presence of old electrons (Baum \& O'Dea, 1990). The question is: Is this diffuse radio emission associated with the young source J0111+3906? Baum et al., (1990) discovered that the brighter peak is located $\approx 18^{\prime \prime}$ east of the core with a diffuse narrow faint bridge of radio emission connecting the extended emission directly back to the nucleus. On the contrary if we compare the angular position of this diffuse emission, Fig.5.14 with the direction of the source in Fig.4.9, we notice that such positions are quite different $\left(\sim 90^{\circ}\right.$ and $\sim 50^{\circ}$ respectively).

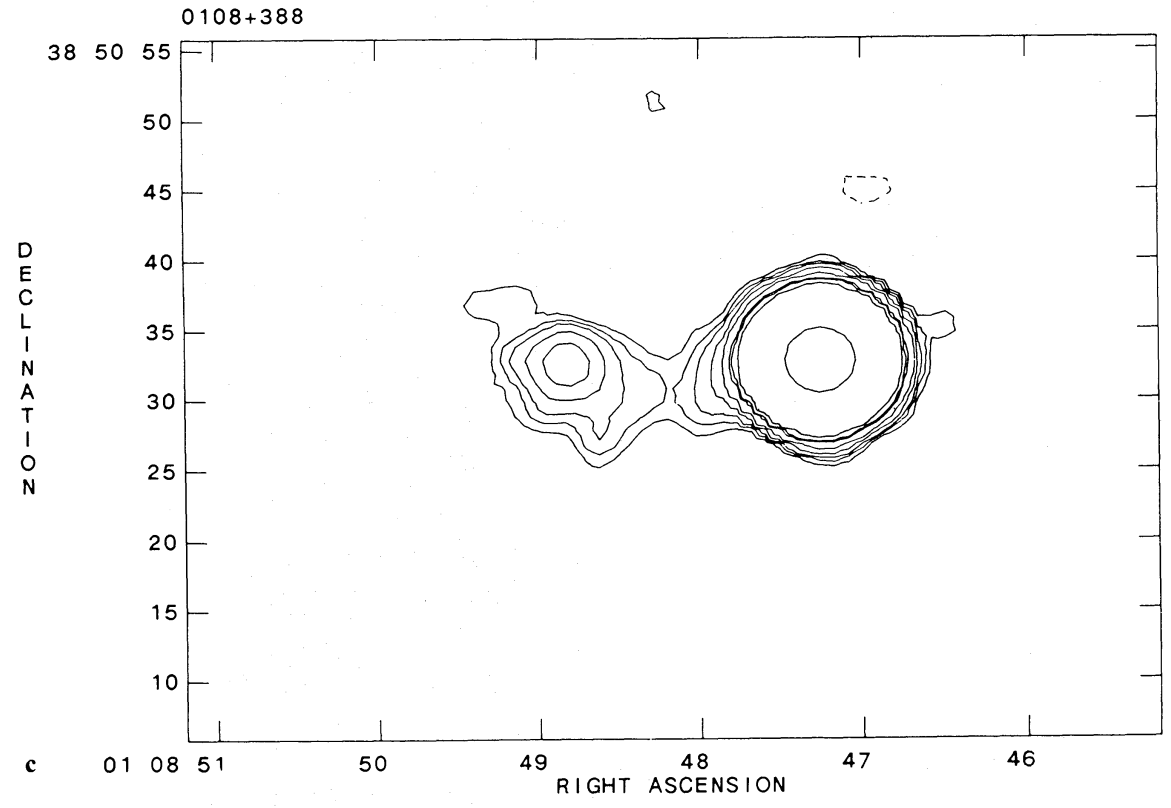

Figure 5.14: The diffuse emission around the radio source J0111+3906 taken with the VLA (Baum \& O’Dea, 1990).

From the different angular position, it seems to be that the compact radio source $\mathrm{J} 0111+3906$ is not connected with the diffuse radio emission observed at low frequencies. This may be interpreted considering that the different orientation between the diffuse radio emission and the new radio structure may be connected with merger events (Czerny et al., 2009). This results in the renewing activity of the central engine accompanied by the re-orientation of the jet axis. By means of this condition, we can explain the different orientation between the new jet orientation and the diffuse radio emission. Therefore, the explanation of this diffuse radio emission is related to the source age and evolution. 
Probably the activity of this object is recurrent and the extended emission is the relic of a previous epoch of activity. The pc-scale in J0111+3906 is very young, but the activity in this source may be intermittent. J0111+3906 may be a so called Re-started Radio Galaxy. 


\section{Conclusions}

Among all radio loud AGN, CSO are compact (LS $<1 \mathrm{kpc}$ ) objects with peculiar properties. They share the same features of Radio loud AGN and, in particular, their morphology is similar to that of FRII, with core, hot spots, jets and lobes. The intrinsically compact morphology of these radio galaxies was debated for a long time. A first model which tried to explain their small size was the so called Frustration Scenario according to which CSO are old radio galaxies completely embedded in a high density ambient medium which prevents their expansion. The frustration scenario is based on the fact that many of these compact sources are found with distorted and asymmetric morphology. This may be due to a anomalous dense ambient medium which prevents the hot spot advance through the IGM resulting in an asymmetric shape of these compact sources. However since no probe of abnormally density in the medium has been discovered, from X-ray and IR observations, this model is not taken into account anymore. On the contrary, the globally accepted scenario that explains the nature of CSO is the Youth Model. According to this model the intrinsically small CSOs are young radio sources, where the radio activity has switched on not long ago. They are still embedded in the ambient medium of the host galaxy and are having a significant interaction with it trying to dig they way out of the galaxy. Following this idea, CSO represents the early stage of evolved $\left(10^{6-7}\right.$ yr) FRII. They will grow and expand over the kpc scale during the radio source lifetime. A number of works appeared in the literature since 1999 provide support to the youth scenario providing ages of the order of $10^{3} \mathrm{yr}$, derived either on the basis of radiative losses or by the VLBI study of source growth.

This model was confirmed by earlier works that found CSO really young with an age of about $10^{3-4} \mathrm{yr}$.

In this thesis work two compact symmetric objects have been studied: J1944+5448 and J0111+3906. Since they are intrinsically small radio sources, they can be observed with the VLBI technique. In particular these two sources, were target of multifrequency ( $\mathrm{L}, \mathrm{C}$ and $\mathrm{X}$ bands) VLBA observations, whose angular resolution allowed us to detect very compact structures. The data have been analyzed by means of the software package NRAO Astronomical Image Processing Software (AIPS) and underwent the operations of 
calibration, editing, cleaning and self calibration. Multifrequency full and low resolution images, and spectral index images were obtained for each source. These provided a fundamental tool to investigate the morphology of these compact radio sources.

The source J1944+5448 is a compact radio galaxy, with a linear size of about $\sim 170$ pc. Two bright hot spots are well visible in the West and East direction, on both the $\mathrm{C}$ and $\mathrm{X}$ band. The core is weak and has a steeper spectrum likely due to some contamination from the jet base. Two lobes are visible in the West and East direction and they are brighter at lower frequencies. Spectral index images confirm the high flux densities found in the lobes and in the core, in $\mathrm{C}$ band In fact at high frequency (X band) there is a steepening in the spectral index of the lobes which is connected with the ageing of the electron population filling the lobes. This produces a break in the radio spectrum which is directly related to the age of the lobes. On the contrary the hot spots are the brightest components of $\mathrm{J} 1944+5448$ at both $\mathrm{C}$ and $\mathrm{X}$ bands and their spectral index is about $\alpha \sim-0.7$ to both these frequencies since they are continuously refreshed with new relativistic particles. J0111+3906 is more compact, with a linear size of about $\sim 40$ pc. In this case two bright hot spots are well detected in the North-East and South-West direction, while the core is weak. A bright jet is clearly visible in the $\mathrm{NE}$ direction.

By estimating the kinematic and spectral age of these sources I derived that J1944+5448 and J0111+3906 are young sources with an age of about $10^{2-3}$ yr. In the case of J1944+5448 I could make a comparison between the kinematic and radiative age and the results were found to be in good agreement.

The estimation of the physical parameters allowed us to derive some additional information on these sources. Since J1944+5448 and J0111+3906 are asymmetric sources, by assuming an equilibrium between the ram pressure and the equipartition pressure the external density of the ambient medium has been estimated. This approach allowed us to understand that the asymmetric shape of both compact radio sources is likely due to the inhomogeneities of the ambient medium in which they are completely embedded. In the case of J1944+5448 the closer hot spot to the core, that is also the brighter one, interacts with an high density medium, which has slowed down the hot spot propagation. Similarly in the case of J0111+3906 the asymmetric shape is due to the dense gas that slow down the hot spot propagation in the NE direction.

In addition to the observation analyzed in this work, archival multi-epochs observations have been considered in order to improve our knowledge about the expansion rate of these compact sources. For J1944+5448 multi-epoch observations in C and X band have been analyzed, considering the works of Polatidis \& Conway (2003) and Xu et al. (1995). The hot spot separation speed has been estimated for each frequency band separately. The results show that J1944+5448 has expanded of about $1.7 \mathrm{pc}$ over the last eighteen years with an expansion rate of about $\sim 0.09 \mathrm{pc} / \mathrm{yr}$. On the contrary J0111+3906 has a lower expansion rate. In this case, in addition to the observation in $\mathrm{C}, \mathrm{X}$ and $\mathrm{U}$ band used 
in this work, also the data available in the literature have been used. Also in this analysis the hot spot separation speed has been found independently for each frequency band. J0111+3906 over the last eighteen years has expanded of only $0.42 \mathrm{pc}$. Therefore, both J1944+5448 and J0111+3906 are compact radio source which are expanding through the ambient medium of the host galaxy.

An important feature of these compact symmetric object is their typically convex radio spectrum. Usually the convex profile of the radio spectrum is correlated to the synchrotron self absorption mechanism that is mainly at work in compact structures. In this thesis work the radio spectrum of the source J0111+3906 was investigated in detail. As a first step, an SSA model was used in order to fit the spectrum of each component of this source. The result about the spectral indices found with the SSA model fit were compared to the spectral index images and they were in good agreement each other. In addition also a free free absorption model was used in order to fit the spectrum of each source component. These result were compared to those found by Marr et al., (2001) who found that the observed turnover in J0111+3906 is due to free free absorption caused by a circum-nuclear disk of ionized gas surrounding the source. On the contrary, our results favour the SSA model as the main mechanism involved in the source J0111+3906. By assuming that the peak is due to the SSA, I estimated the magnetic field and it turned out to be consistent with the equipartition magnetic field. The source J0111+3906 has a high frequency turnover, around $5 \mathrm{GHz}$, and thus it may be classify as a HFP according to the spectral classification (Dallacasa et al., 2000).

In conclusion this thesis work allows us to derive that:

- J1944+5448 and J0111+3906 are compact and young radio sources with and age of about $10^{2-3}$ yr. This confirms the youth scenario according to which CSO represents the early stage of evolved Radio loud AGN (mainly FRII).

- The compact source, J0111+3906 (HFP) is also younger than J1944+5448 and consequently it has a higher frequency turnover $(\sim 5 \mathrm{GHz})$. This agrees with the idea that HFP represent the very early stage of evolved radio galaxy, and in these compact radio source probably the radio activity has switched on only recently.

- J1944+5448 and J0111+3906 are expanding with an expansion rate ranging between $(\sim 0.09$ and $\sim 0.02) \mathrm{pc} / \mathrm{yr}$, respectively, through the ambient medium of the host galaxy.

- The asymmetries found in the morphology of these sources may be explained considering the inhomogeneities of the gas embedding the sources. In the case of 
$\mathrm{J} 1944+5448$ the density of the medium is $\sim 15$ times larger in the West part than in the East one, and similarly on the case of J0111+3906 the density in the North-East direction is $\sim 10$ times greater than in the opposite side.

In fact, the high density medium may slow down the hot spot advance speed and produce the asymmetric shape of these compact symmetric objects.

- The radio spectrum of J0111+3906 may be well fitted by a synchrotron self absorption model according to which the turnover of this radio galaxy occurs at high frequency $(4 \mathrm{GHz})$. A free free self absorption model, was also fitted but provided a worse result, however on the basis of the spectral analysis we cannot exclude it. Our results favour an SSA model, but a combination of these two mechanisms may be at work in J0111+3906.

It is worth mentioning that additional multifrequency observations would help the improvement of our knowledge of these particularly objects. In particular, for investigating their morphological and spectral features. Moreover new epochs of observations would reduce the uncertainty on the estimation of the hot spot separation speed and would allow a better estimation of the kinematic age of these sources. Therefore a frequent monitoring of CSO is a fundamental tool to investigate the evolution of these compact and young AGN, and in addition their analysis is fundamental in improving the knowledge on the evolution of radio loud active galactic nuclei. 
The results of this thesis work were presented in a contributed talk at the "5th Workshop on Compact Steep Spectrum and Gigahertz-Peaked Spectrum radio sources" in Rimini, May 27-29 2015: "Multifrequency $V L B A$ observations of two compact symmetric objects. The referred proceedings will be published in Astronomische Nachrichten (Astronomical Notes). 



\section{Appendix A}

\section{Physical parameters in radio sources}

In this appendix I briefly explain how I estimated the physical parameters in radio sources, such as the luminosity, the energy and the magnetic field.

The integrated Luminosity (L) between $10 \mathrm{MHz}$ and $100 \mathrm{GHz}$, of a radio source can be estimated according to:

$$
\mathrm{L}=4 \pi \mathrm{D}_{\mathrm{L}}^{2}(1+\mathrm{z})^{(\alpha-1)} \int_{10 \mathrm{MHz}}^{100 \mathrm{GHZ}} \mathrm{S}(\nu) \mathrm{d} \nu
$$

where $\mathrm{D}_{\mathrm{L}}$ is the luminosity distance, $\mathrm{z}$ is the redshift, $\alpha$ is the spectral index and $\mathrm{S}(\nu)$ is the flux density at frequency $\nu$ described by a power law as follows:

$$
\mathrm{S}(\nu)=\mathrm{S}_{0}\left(\frac{\nu}{\nu_{0}}\right)^{\alpha}
$$

where $\mathrm{S}_{0}$ is the flux density at the frequency $\nu_{0}$ and $\alpha$ is the spectral index. In this case It is convenient, assuming $\alpha=-0.7$ which is a typical value for a radio source. The quantity $(1+z)^{(-\alpha+1)}$ is called $K$-correction and it takes into consideration the frequency shift of the emission between the target rest-frame and the observer frame.

The total energy radiated from an object which emits by synchrotron radiation can be expressed by:

$$
\mathrm{E}_{\mathrm{tot}}=\mathrm{E}_{\mathrm{el}}+\mathrm{E}_{\mathrm{pr}}+\mathrm{E}_{\mathrm{H}}
$$


where $E_{e l}$ is the energy content of the electron populations, $E_{p r}$ is the energy content of the protons and $\mathrm{E}_{\mathrm{H}}$ is the energy of the magnetic field. The energy contained in the heavy particles, $\mathrm{E}_{\mathrm{pr}}$, can be related to $\mathrm{E}_{\mathrm{el}}$ assuming:

$$
\mathrm{E}_{\mathrm{pr}}=\mathrm{k} \times \mathrm{E}_{\mathrm{el}}
$$

and then we have:

$$
\mathrm{E}_{\mathrm{el}}+\mathrm{E}_{\mathrm{pr}}=(1+\mathrm{k}) \times \mathrm{E}_{\mathrm{el}}
$$

thus considering the proton energy proportional to the electron energy with $\mathrm{k}>0$. Without going in detail, assuming that the energy distribution of the electrons is

$$
\mathrm{N}(\mathrm{E})=\mathrm{N}_{0} \mathrm{E}^{-\delta}
$$

(as discussed in Chapter 2) we can derive the energy stored in the relativistic particles. which is related to the luminosity that is an observable quantity and then to the magnetic field $\mathrm{H}$.

$$
\mathrm{E}_{\mathrm{el}}=\mathrm{V} \int \mathrm{EN}(\mathrm{E}) \mathrm{dE}=\mathrm{C}_{\mathrm{el}} \mathrm{H}^{-3 / 2} \mathrm{~L}
$$

where $\mathrm{V}$ is the volume of the source, $\mathrm{N}(\mathrm{E})$ the energy distribution, $\mathrm{L}$ is the luminosity, $\mathrm{H}$ is the magnetic field and $\mathrm{C}_{\mathrm{el}}$ is a constant which depends weakly on the spectral index $\alpha$ and on the energy range of the relativistic particle distribution.

The magnetic field electrons are assumed uniform over the volume $\mathrm{V}$, in particular the plasma in which the magnetic field is frozen fills uniformly the volume $\mathrm{V}$ with a filling factor $\phi=1$.

Then we can derive the magnetic field energy. which is:

$$
\mathrm{E}_{\mathrm{H}}=\frac{1}{8 \pi} \int \mathrm{H}^{2} \mathrm{dV}=\mathrm{C}_{\mathrm{M}} \mathrm{H}^{2} \mathrm{~V}
$$

where $\mathrm{C}_{\mathrm{M}}$ is a constant. Therefore, according to Eq.A.8 the total energy contained in a radio source is:

$$
\mathrm{E}_{\mathrm{tot}}=(1+\mathrm{k}) \times \mathrm{C}_{\mathrm{el}} \mathrm{H}^{-3 / 2} \mathrm{~L}+\mathrm{C}_{\mathrm{M}} \mathrm{H}^{2} \mathrm{~V}
$$

It depends on three main parameters such as the volume, the luminosity and the magnetic field. 
The magnetic field may be estimated by assuming the total minimum energy condition which occurs for

$$
\frac{\partial \mathrm{E}_{\mathrm{tot}}}{\partial \mathrm{H}}=0
$$

For this minimum value we have that

$$
(1+\mathrm{k}) \mathrm{E}_{\mathrm{el}}=\frac{4}{3} \mathrm{E}_{\mathrm{H}}
$$

that is also called the Equipartition condition in which there is rough equipartition between the energy contained in the particles and in the magnetic field. The total energy $E_{\text {tot }}$ is

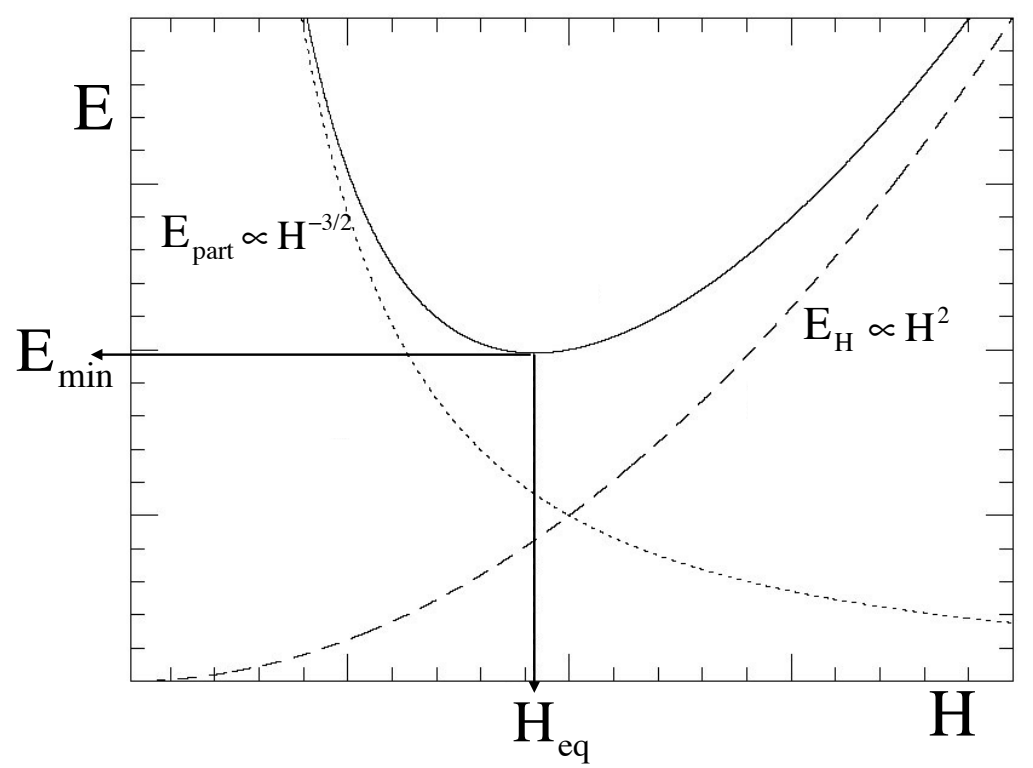

Figure A.1: The minimum energy condition which corresponds to the equipartition of the energy in a radio source.

$$
\mathrm{E}_{\mathrm{tot}}=(1+\mathrm{k})^{4 / 7} \mathrm{c}_{\mathrm{el}}^{4 / 7} \mathrm{~L}^{4 / 7} \mathrm{~V}^{3 / 7}
$$

while the minimum energy density $u_{\min }$ is

$$
\mathrm{u}_{\min }=\frac{\mathrm{E}_{\mathrm{tot}}}{\mathrm{V}}=(1+\mathrm{k})^{4 / 7} \mathrm{cel}_{\mathrm{el}}^{4 / 7} \mathrm{~L}^{4 / 7} \mathrm{~V}^{-4 / 7}
$$

Then according to Eq. A.9 and Eq. A.8 we can estimate the so called equipartition magnetic field $\mathrm{H}_{\mathrm{eq}}$ (Pacholczyk, 1970) as follows:

$$
\mathrm{H}_{\mathrm{eq}}=(4.5)^{2 / 7}(1+\mathrm{k})^{2 / 7} \mathrm{c}_{\mathrm{el}}^{2 / 7} \mathrm{~L}^{2 / 7} \mathrm{~V}^{-2 / 7}
$$

In this equation $c_{\mathrm{el}}$ is a tabulated value and depends on the frequency and $\delta$. In our case we used $c_{\mathrm{el}}=2.7 \times 10^{7}$. In addition, we assumed $\mathrm{k}=1$ that is $\mathrm{E}_{\mathrm{el}}=\mathrm{E}_{\mathrm{pr}}$. 
Finally we can also derive the equipartition pressure, $\mathrm{p}_{\mathrm{eq}}$, from the radiation and magnetic energy density, which is:

$$
\mathrm{p}_{\text {eq }}=\frac{11}{21} \mathrm{u}_{\mathrm{min}}
$$




\section{Bibliography}

1995, VLBI Observing Strategies, Vol. 82

Antonucci, R. 1993, ARA\&A, 31, 473

Artyukh, V. S., Chernikov, P. A., \& Tyul'Bashev, S. A. 2008, A\&A, 486, 735

Baum, S. A., \& O'Dea, C. P. 1990, A\&AS, 232, 19

Bicknell, C. V., Dopita, M. A., \& O'Dea, C. P. 1997a, in Astronomical Society of the Pacific Conference Series, Vol. 121, IAU Colloq. 163: Accretion Phenomena and Related Outflows, ed. D. T. Wickramasinghe, G. V. Bicknell, \& L. Ferrario, 672

Bicknell, G. V., \& Dopita, M. A. 1997, in Relativistic Jets in AGNs, ed. M. Ostrowski, M. Sikora, G. Madejski, \& M. Begelman, 1-12

Bicknell, G. V., Dopita, M. A., \& O'Dea, C. P. 1997b, in Astronomical Society of the Pacific Conference Series, Vol. 113, IAU Colloq. 159: Emission Lines in Active Galaxies: New Methods and Techniques, ed. B. M. Peterson, F.-Z. Cheng, \& A. S. Wilson, 382

Carvalho, J. C. 1994, A\&A, 292, 392

-. 1998, A\&A, 329, 845

Cornwell, T., \& Fomalont, E. B. 1999, in

Cotton, W. D. 1995, in Very Long Baseline Interferometry and the VLBA, Vol. 82, 189

Cotton, W. D., Dallacasa, D., Fanti, C., et al. 2003, PASA, 20, 12

Czerny, B., Siemiginowska, A., Janiuk, A., Nikiel-Wroczyński, B., \& Stawarz, Ł. 2009, ApJ, 698, 840

Dallacasa, D. 2003, PASA, 20, 79

Dallacasa, D., Stanghellini, C., Centonza, M., \& Fanti, R. 2000, A\&A, 363, 887

De Young, D. S. 1993, ApJ, 402, 95

Dopita, M. A., Bicknell, G. V., \& O'Dea, C. P. 1997, in Gigahertz Peaked Spectrum and Compact Steep Spectrum Radio Sources, ed. I. A. G. Snellen, R. T. Schilizzi, H. J. A. Roettgering, \& M. N. Bremer, 229-240 
Fanaroff, B. L., \& Riley, J. M. 1974, MNRAS, 167, 31P

Fanti, C., \& Fanti, R. 1999-2000

Fanti, C., Fanti, R., Dallacasa, D., et al. 1995, A\&A, 302, 317

Fanti, C., Fanti, R., \& O'Dea, C. P., eds. 1990, Compact steep-spectrum and GHZ-peaked spectrum radio sources

Fanti, C., Pozzi, F., Fanti, R., et al. 2000, A\&A, 358

Fanti, R. 2004, in The Role of VLBI in Astrophysics, Astrometry and Geodesy, ed. F. Mantovani \& A. Kus, 157

Jaffe, W. J., \& Perola, G. C. 1973, A\&A, 26, 423

Kardashev, N. S. 1962, Soviet Ast., 6, 317

Krolik, J. H., \& Pier, E. A. 1992, Bulk relativistic motion in gamma-ray bursts, ed. C. Ho, R. I. Epstein, \& E. E. Fenimore, 99-106

Lister, M. 2003, in Bulletin of the American Astronomical Society

Lister, M. L., Aller, H. D., Aller, M. F., et al. 2009, AJ, 137

Lister, M. L., Aller, M. F., Aller, H. D., et al. 2013, AJ, 146

Marr, J., \& Taylor, G. B. 2001, News Letter of the Astronomical Society of New York, 5, 6

Marr, J. M., Crawford, III, F., \& Taylor, G. B. 1998, in IAU Symposium, Vol. 184, The Central Regions of the Galaxy and Galaxies, ed. Y. Sofue, 401

Marr, J. M., Taylor, G. B., \& Crawford, III, F. 2000, in Astrophysical Phenomena Revealed by Space VLBI, ed. H. Hirabayashi, P. G. Edwards, \& D. W. Murphy, 91-94

Marr, J. M., Taylor, G. B., \& Crawford, III, F. 2001, ApJ, 550, 160

Meliani, Z., Sauty, C., Tsinganos, K., Trussoni, E., \& Cayatte, V. 2010, A\&A, 521, A67

Mezger, P. G., \& Henderson, A. P. 1967, ApJ, 147

Moffett, A. T. 1975, IX

Murgia, M. 2003, PASA, 20, 19

Murgia, M., Fanti, C., Fanti, R., et al. 1999, A\&A, 345, 769

Nagai, H., Inoue, M., Asada, K., Kameno, S., \& Doi, A. 2006, ApJ, 648, 148

O’Dea, C. P. 1998, PASP, 110, 493

O’Dea, C. P., \& Baum, S. A. 1997, AJ, 113 
O'Dea, C. P., Baum, S. A., \& Stanghellini, C. 1991, ApJ, 380, 66

Orienti, M. 2007, PhD thesis

Orienti, M., \& Dallacasa, D. 2008a, A\&A, 487, 885

Orienti, M., \& Dallacasa, D. 2008b, in Astronomical Society of the Pacific Conference Series, Vol. 386, Extragalactic Jets: Theory and Observation from Radio to Gamma Ray, ed. T. A. Rector \& D. S. De Young, 196

一. 2013, MNRAS, 438

Orienti, M., Dallacasa, D., \& Stanghellini, C. 2007, A\&A, 475, 813

Orienti, M., Dallacasa, D., Tinti, S., \& Stanghellini, C. 2006, A\&A, 450, 959

Orienti, M., Venturi, T., Dallacasa, D., et al. 2011, MNRAS, 417, 359

Owen, F. N., \& Ledlow, M. J. 1994, in Astronomical Society of the Pacific Conference Series, Vol. 54, The Physics of Active Galaxies, ed. G. V. Bicknell, M. A. Dopita, \& P. J. Quinn, 319

Owsianik, I., Conway, J. E., \& Polatidis, A. G. 1998, A\&A, 336, L37

Pacholczyk, A. G. 1970, Radio astrophysics. Nonthermal processes in galactic and extragalactic sources

Peterson, B. 1997a, An Introduction to Active Galactic Nuclei, ed. C. U. Press

-. 2006, Host Galaxies of Reverberation Mapped AGNs, HST Proposal

Peterson, B. M. 1993, PASP, 105, 247

—. 1997b, An Introduction to Active Galactic Nuclei

Peterson, B. M., Ali, B., Horne, K., et al. 1993, ApJ, 402, 469

Phillips, R. B., \& Mutel, R. L. 1982, A\&A, 106, 21

Polatidis, A., Wilkinson, P. N., Xu, W., et al. 1999, New A Rev., 43, 657

Polatidis, A. G. 2003, Astronomische Nachrichten Supplement, 324, 25

Polatidis, A. G., \& Conway, J. E. 2003, PASA, 20, 69

Readhead, A. C. S. 1995, Proceedings of the National Academy of Science, 92, 11447

Saikia, D. J., Jeyakumar, S., Mantovani, F., et al. 2003, PASA, 20, 50

Scheuer, P. A. G. 1982, in IAU Symposium, Vol. 97, Extragalactic Radio Sources, ed. D. S. Heeschen \& C. M. Wade, 163-165

Shakura, N. I., \& Sunyaev, R. A. 1973, A\&A, 24, 337 
Siemiginowska, A., Cheung, C. C., LaMassa, S., et al. 2005, ApJ, 632, 110

Snellen, I. A. G., Schilizzi, R. T., de Bruyn, A. G., et al. 1998, A\&AS, 131, 435

Snellen, I. A. G., Schilizzi, R. T., Miley, G. K., et al. 2000a, MNRAS, 319, 445

Snellen, I. A. G., Schilizzi, R. T., \& van Langevelde, H. J. 2000b, MNRAS, 319, 429

Stanghellini, C. 2003, PASA, 20, 118

Stanghellini, C., Dallacasa, D., O’Dea, C. P., et al. 2001, A\&A, 377, 377

Stanghellini, C., Dallacasa, D., \& Orienti, M. 2009, Astronomische Nachrichten, 330, 223

Taylor, G. B., Marr, J. M., Pearson, T. J., \& Readhead, A. C. S. 2000, ApJ, 541, 112

Thompson, A., Moran, J., \& Swenson, G. 2001, Interferometry and synthesis in radio astronomy, ed. Wiley- $\mathrm{VCH}$

Tinti, S., Dallacasa, D., de Zotti, G., Celotti, A., \& Stanghellini, C. 2005, A\&A, 432, 31

Tinti, S., Dallacasa, D., De Zotti, G., Stanghellini, C., \& Celotti, A. 2003, ArXiv Astrophysics e-prints, astro-ph/0309354

Trinchieri, G., Fabbiano, G., \& Canizares, C. R. 1986, ApJ, 310, 637

Urry, C. M., \& Padovani, P. 1995, PASP, 107, 803

van Breugel, W. J. M., Miley, G., \& Heckman, T. 1984, AJ, 89

Wagner, A. Y., Bicknell, G. V., \& Umemura, M. 2012, ApJ, 757, 136

Walker, R. C. 1995, in Practical VLBI Imaging, Vol. 82

Wilkinson, P. N., Spencer, R. E., Readhead, A. C. S., Pearson, T. J., \& Simon, R. S. 1984, in IAU Symposium, Vol. 110, VLBI and Compact Radio Sources, ed. R. Fanti, K. I. Kellermann, \& G. Setti, 25

Xu, W., Readhead, A. C. S., Pearson, T. J., Polatidis, A. G., \& Wilkinson, P. N. 1995, ApJS, 99, 297 